\title{
Catalytic Asymmetric Synthesis of Chiral Allylic Amines. Evaluation of Ferrocenyloxazoline Palladacycle Catalysts and Imidate Motifs
}

Carolyn E. Anderson, Yariv Donde, Christopher J. Douglas and Larry E. Overman*

Department of Chemistry, 516 Rowland Hall, University of California, Irvine, CA 92697-2025

\section{Supporting Information}

(63 Pages)

Section A contains representative experimental procedures for the preparation of $\mathbf{5 c}-\mathbf{d}$, 6c-d, 26a-h, 27a-c, 27e-f, 27h, 29-36, 43c-d, 45-47, 49, 50, 53 with tabulated characterization data for these new compounds. Pages S2-S23

Section B contains copies of ${ }^{1} \mathrm{H}$ and ${ }^{13} \mathrm{C}$ NMR spectra for new compounds described in section A. Pages S24-S57

Section C contains representative HPLC traces for selected reactions. Pages S58-S63 


\section{A. Experimental Procedures and Tabulated Characterization Data}

General experimental details: The procedure employed to purify $\mathrm{THF}$ and $\mathrm{CH}_{2} \mathrm{Cl}_{2}$ has been described; ${ }^{1}$ other general experimental details also have been described. ${ }^{2}$

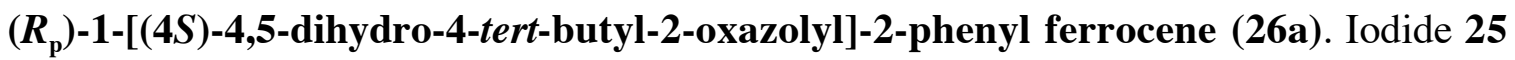

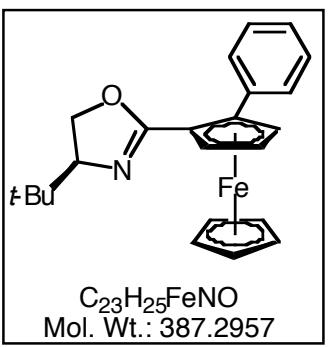

(300 mg, $0.69 \mathrm{mmol}$ ), phenylboronic acid (167 mg, $1.3 \mathrm{mmol}$ ), and $\mathrm{PdCl}_{2} \mathrm{dppf} \cdot \mathrm{CH}_{2} \mathrm{Cl}_{2}(56 \mathrm{mg}, 0.07 \mathrm{mmol})$ were charged to a sealable reaction flask. The flask was evacuated and backfilled with argon before adding DME (3.5 mL) and aqueous $\mathrm{NaOH}(3 \mathrm{~N}, 0.56 \mathrm{~mL}$, $1.73 \mathrm{mmol})$. The resulting black suspension was sparged with argon

for $15 \mathrm{~min}$. The reaction was heated to $85^{\circ} \mathrm{C}$ and maintained for $2 \mathrm{~h}$ before cooling to room temperature. The reaction was then diluted with EtOAc $(50 \mathrm{~mL})$ and extracted with water $(50 \mathrm{~mL})$. The aqueous phase was extracted with EtOAc $(50 \mathrm{~mL})$ and the combined organic portions were washed with brine $(50 \mathrm{~mL})$, dried $\left(\mathrm{MgSO}_{4}\right)$, and concentrated in vacuo to afford a red oil. Purification by column chromatography $\left(\mathrm{CH}_{2} \mathrm{Cl}_{2}\right)$ provided $\mathbf{2 6 a}$ $(224 \mathrm{mg}, 84 \%)$ as an orange foam: $[\square]_{\mathrm{D}}{ }^{26}=-8.8^{\circ},[\square]_{577}{ }^{26}=-10.1^{\circ},[\square]_{546}{ }^{26}=-16.0^{\circ},(\mathrm{c}=$ 0.9, $\left.\mathrm{CHCl}_{3}\right) ;{ }^{1} \mathrm{H}$ NMR $\left(500 \mathrm{MHz}, \mathrm{C}_{6} \mathrm{D}_{6}\right) \square 7.94(\mathrm{~d}, J=7.3 \mathrm{~Hz}, 2 \mathrm{H}), 7.34(\mathrm{t}, J=7.4 \mathrm{~Hz}$, 2H), $7.25(\mathrm{~m}, 1 \mathrm{H}),{ }^{20} 5.06$ (app s, 1H), 4.47 (app s 1H), 4.19 (s, 5H), 3.80-3.89 (m, 3H), 0.98 (s, 9H); ${ }^{13} \mathrm{C}$ NMR (125 MHz, $\left.\mathrm{C}_{6} \mathrm{D}_{6}\right) \square 164.3,137.8,130.6,127.2,126.7,89.6,76.9$, 72.8, 71.6, 71.3, 70.5, 69.2, 67.8, 33.9, 26.3; IR (film) 2955, 1659, $1602 \mathrm{~cm}^{-1}$; LRMS (ES) $m / z 410(\mathrm{M}+\mathrm{Na})^{+}, 388(\mathrm{M}+\mathrm{H})^{+}$; Anal. Calcd for $\mathrm{C}_{23} \mathrm{H}_{25} \mathrm{FeNO}$ : C, 71.33; H, 6.51; N, 3.62. Found: C, 71.19; H, 6.57; N, 3.52.

\footnotetext{
${ }^{1}$ Pangborn, A. B.; Giardello, M. A.; Grubbs, R. H.; Rosen, R. K.; Timmers, F. J. Organometallics, 1996, $15,1518$.

${ }^{2}$ Deng, W.; Overman, L. E. J. Am. Chem. Soc. 1994, 116, 11241.
} 


\section{$\left(R_{\mathrm{p}}\right)-1-[(4 S)-4,5-d i h y d r o-4-t e r t-b u t y l-2-o x a z o l y l]-2-(4-m e t h o x y p h e n y l)$ ferrocene}

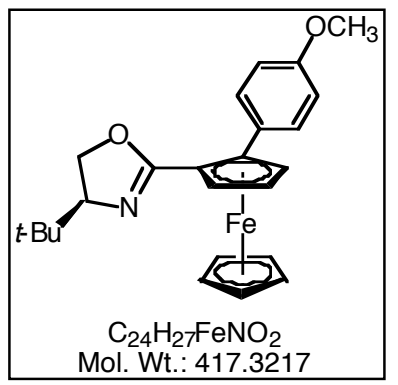

(26b). According to the procedure for the preparation of 26a, 4methoxyphenylboronic acid (148 $\mathrm{mg}, 0.92 \mathrm{mmol}$ ) and iodide 25 (200 mg, $0.46 \mathrm{mmol}$ ) were condensed to afford a red oil which upon purification by column chromatography (1:99, $\mathrm{Et}_{2} \mathrm{O} / \mathrm{CH}_{2} \mathrm{Cl}_{2}$ ) provided 26b (169 $\mathrm{mg}, 88 \%$ ) as an orange foam: $[\square]_{\mathrm{D}}{ }^{26}=-12.4^{\circ},[\square]_{577}{ }^{26}=-14.1^{\circ},[\square]_{546}{ }^{26}=-22.5^{\circ},(c=0.9$,

$\mathrm{CHCl}_{3}$ ); ${ }^{1} \mathrm{H} \mathrm{NMR}\left(500 \mathrm{MHz}, \mathrm{C}_{6} \mathrm{D}_{6}\right) \square 7.90$ (app dt, $\left.J=8.9,2.2 \mathrm{~Hz}, 2 \mathrm{H}\right), 6.96$ (app dt, $J=$ 8.8, 2.6 Hz, 2H), 5.07 (dd, $J=2.6,1.7 \mathrm{~Hz}, 1 \mathrm{H}), 4.48(\mathrm{dd}, J=2.4,1.7 \mathrm{~Hz}, 1 \mathrm{H}), 4.23$ (s, $5 \mathrm{H}), 4.20(\operatorname{app~t}, J=2.6 \mathrm{~Hz}, 1 \mathrm{H}), 3.90-3.92(\mathrm{~m}, 2 \mathrm{H}), 3.83(\mathrm{dd}, J=10.2,7.8 \mathrm{~Hz}, 1 \mathrm{H})$, $3.46(\mathrm{~s}, 3 \mathrm{H}), 1.00(\mathrm{~s}, 9 \mathrm{H}) ;{ }^{13} \mathrm{C} \mathrm{NMR}\left(125 \mathrm{MHz}, \mathrm{C}_{6} \mathrm{D}_{6}\right) \square 164.5,158.8,131.6,129.7$, 112.9, 89.7, 76.9, 72.5, 71.3, 71.2, 70.2, 69.0, 67.8, 54.9, 33.9, 26.3; IR (film) 2955, 1660, $1612 \mathrm{~cm}^{-1}$; LRMS (CI, isobutane) $\mathrm{m} / z 417(\mathrm{M})^{+}, 360(\mathrm{M}-t \text {-Bu})^{+}, 317(\mathrm{M}$ -methoxyphenyl) ${ }^{+}$; Anal. Calcd for $\mathrm{C}_{24} \mathrm{H}_{27} \mathrm{FeNO}_{2}$ : C, 69.07; H, 6.52; N, 3.36. Found: C, 69.28; H, 6.39; N, 3.09.

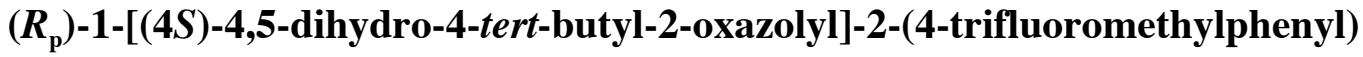

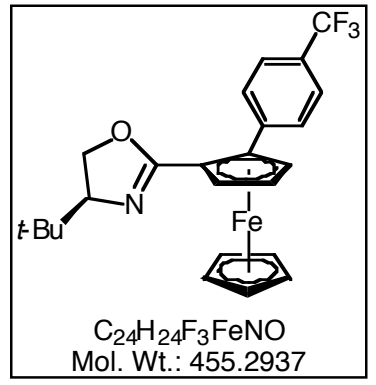

ferrocene (26c). According to the procedure for the preparation of 26a, 4-trifluoromethylphenylboronic acid (1.9 g, $10.0 \mathrm{mmol})$ and iodide 25 (1.09 g, $2.5 \mathrm{mmol})$ were condensed to afford a red oil which upon purification by column chromatography $(5: 95$, $\mathrm{Et}_{2} \mathrm{O} / \mathrm{CH}_{2} \mathrm{Cl}_{2}$ ) and recrystallization ( $n$-hexane, $-20{ }^{\circ} \mathrm{C}$ ) gave 26c

(351 mg, 33\%) as orange prisms: mp $132-135{ }^{\circ} \mathrm{C}$; $[\square]_{\mathrm{D}}{ }^{26}=-14.8^{\circ},[\square]_{577}{ }^{26}=-16.9^{\circ}$, $[\square]_{546}{ }^{26}=-25.2^{\circ},\left(c=2.2, \mathrm{CHCl}_{3}\right) ;{ }^{1} \mathrm{H} \mathrm{NMR}\left(400 \mathrm{MHz}, \mathrm{C}_{6} \mathrm{D}_{6}\right) \square 7.74(\mathrm{~d}, J=8.0 \mathrm{~Hz}, 2 \mathrm{H})$, $7.42(\mathrm{~d}, J=8.1 \mathrm{~Hz}, 2 \mathrm{H}), 4.94(\mathrm{dd}, J=2.6,1.6 \mathrm{~Hz}, 1 \mathrm{H}), 4.24(\mathrm{dd}, J=2.5,1.6 \mathrm{~Hz}, 1 \mathrm{H})$, 4.07 (app t, $J=2.6 \mathrm{~Hz}, 1 \mathrm{H}), 4.02(\mathrm{~s}, 5 \mathrm{H}), 3.74-3.72(\mathrm{~m}, 2 \mathrm{H}), 3.66$ (dd, $J=10.7,7.0 \mathrm{~Hz}$, $1 \mathrm{H}), 0.83(\mathrm{~s}, 9 \mathrm{H}) ;{ }^{13} \mathrm{C} \mathrm{NMR}\left(125 \mathrm{MHz}, \mathrm{CDCl}_{3}\right) \square 164.6,141.8,130.3,128.4$ (q, $J=32.3$ $\mathrm{Hz}), 124.4(\mathrm{q}, J=270.3 \mathrm{~Hz}), 124.0(\operatorname{app~q}, \mathrm{J}=3.5 \mathrm{~Hz}), 86.8,76.6,72.2,71.7,71.1,70.0$, 69.4, 67.9, 33.8, 26.0; IR (film) 3054, 2961, 1659, $1616 \mathrm{~cm}^{-1}$; LRMS (ES) $\mathrm{m} / z .78$ (M + 
$\mathrm{Na})^{+}, 456(\mathrm{M}+\mathrm{H})^{+}$; Anal. Calcd for $\mathrm{C}_{24} \mathrm{H}_{24} \mathrm{~F}_{3} \mathrm{FeNO}$ : C, 63.31; H, 5.31; N, 3.08. Found: C, 63.38; H, 5.52; N, 2.95 .

$\left(R_{\mathrm{p}}\right)-1-[(4 S)-4,5-d i h y d r o-4-t e r t-b u t y l-2-0 x a z o l y l]-2-(2,6-d i m e t h o x y p h e n y l)$ ferrocene

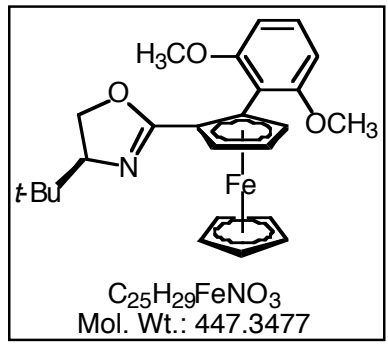

Mol. Wt.: 447.3477 (26d). According to the procedure for the preparation of $\mathbf{2 6 a}$, 2,6-dimethoxyphenylboronic acid (546 $\mathrm{mg}, 3.0 \mathrm{mmol})$ and iodide 25 (658 mg, $1.5 \mathrm{mmol})$ were condensed to afford a red oil which upon purification by column chromatography $(5: 95$, $\mathrm{Et}_{2} \mathrm{O} / \mathrm{CH}_{2} \mathrm{Cl}_{2}$ ) and recrystallization (pentane, $-20{ }^{\circ} \mathrm{C}$ ) afforded

26d (134 mg, 20\%) as orange needles: $\mathrm{mp} 147-149{ }^{\circ} \mathrm{C}$; $[\square]_{\mathrm{D}}{ }^{26}=+37.3^{\circ},[\square]_{577}{ }^{26}=+47.4^{\circ}$, $[\square]_{546}{ }^{26}=+79.1^{\circ},[\square]_{435}{ }^{26}=-241.7^{\circ},[\square]_{405}{ }^{26}=-312.8^{\circ},\left(\mathrm{c}=0.1, \mathrm{CHCl}_{3}\right) ;{ }^{1} \mathrm{H}$ NMR $(500$ $\left.\mathrm{MHz} \mathrm{CDCl}_{3}\right) \square 7.90(\mathrm{t}, J=8.3 \mathrm{~Hz}, 1 \mathrm{H}), 6.55(\mathrm{~d}, J=8.3 \mathrm{~Hz}, 2 \mathrm{H}), 4.90($ app t, $J=2.0 \mathrm{~Hz}$, $1 \mathrm{H}), 4.43-4.44$ (m, 2H), 4.18 (s, 5H), 4.15 (dd, $J=8.3,7.2 \mathrm{~Hz}, 1 \mathrm{H}), 3.74-3.83$ (m, 8H), 0.94 (s, 9H); ${ }^{13} \mathrm{C}$ NMR (125 MHz, $\left.\mathrm{CDCl}_{3}\right) \square 167.1,158.2$ (b), 127.6, 113.9, 103.9, 80.4, 75.9, 73.7, 71.1, 70.8, 68.9, 68.6, 68.4, 55.8, 33.2, 26.4; IR (film) 2953, 1645, $1586 \mathrm{~cm}^{-1}$; LRMS (ES) $m / z 470(\mathrm{M}+\mathrm{Na})^{+}, 448(\mathrm{M}+\mathrm{H})^{+}$; Anal. Calcd for $\mathrm{C}_{25} \mathrm{H}_{29} \mathrm{FeNO}_{3}$ : C, 67.12; H, 6.53; N, 3.13. Found: C, 67.34; H, 6.60; N, 3.06.

$\left(R_{\mathrm{p}}\right)-1-[(4 S)-4,5-d i h y d r o-4-t e r t-b u t y l-2-o x a z o l y l)-2-(1-n a p t h y l]$ ferrocene (26e).

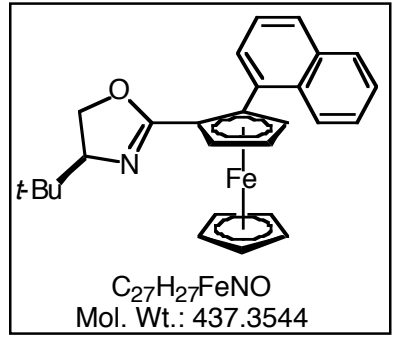
According to the procedure for the preparation of $\mathbf{2 6 a}$, iodide $\mathbf{2 5}$ (876 mg, $2.00 \mathrm{mmol})$ and 1-naphthaleneboronic acid (688 mg, $4.00 \mathrm{mmol})$ were condensed to afford a red oil. Purification of this oil by column chromatography $\left(\mathrm{CH}_{2} \mathrm{Cl}_{2}\right)$ and recrystallization ( $n$-hexane, $-20{ }^{\circ} \mathrm{C}$ ) provided 26e $(802 \mathrm{mg}$, 92\%) as yellow needles: mp 107-109 ${ }^{\circ} \mathrm{C}$; $[\square]_{\mathrm{D}}{ }^{26}=+21.1^{\circ},[\square]_{577}{ }^{26}=+24.3^{\circ},[\square]_{546}{ }^{26}=$ $+37.8^{\circ},[\square]_{435}{ }^{26}=-42.7^{\circ},[\square]_{405}{ }^{26}=-68.8^{\circ},\left(c=1.0, \mathrm{CHCl}_{3}\right) ;{ }^{1} \mathrm{H} \mathrm{NMR}\left(500 \mathrm{MHz}, \mathrm{C}_{6} \mathrm{D}_{6}\right) \square$ $8.32(\mathrm{~d}, J=7.0 \mathrm{~Hz}, 1 \mathrm{H}), 8.01(\mathrm{~d}, J=8.2 \mathrm{~Hz}, 1 \mathrm{H}), 7.87(\mathrm{t}, J=7.1 \mathrm{~Hz}, 2 \mathrm{H}), 7.55(\mathrm{t}, J=7.7$ $\mathrm{Hz}, 1 \mathrm{H}), 7.27-7.33(\mathrm{~m}, 2 \mathrm{H}), 5.25(\operatorname{app~t}, J=2.0 \mathrm{~Hz}, 1 \mathrm{H}), 4.38($ app t, $J=1.8 \mathrm{~Hz}, 1 \mathrm{H})$, $4.32(\mathrm{~s}, 5 \mathrm{H}), 4.21(\operatorname{app~t}, J=2.4 \mathrm{~Hz}, 1 \mathrm{H}), 3.61-3.68(\mathrm{~m}, 2 \mathrm{H}), 3.36-3.42(\mathrm{~m}, 1 \mathrm{H}), 0.87$ (s, 
$9 \mathrm{H}) ;{ }^{13} \mathrm{C}$ NMR $\left(125 \mathrm{MHz}, \mathrm{CDCl}_{3}\right) \square 165.1,134.6,133.3,133.0,129.3,128.0,127.3$, $125.9,125.5,125.1,124.8,89.9,76.0,75.0,71.5,70.8,69.3,68.7,68.1,33.5,26.0$; IR (film) 3054, 2986, 1646, $1598 \mathrm{~cm}^{-1}$; LRMS (ES) m/z $460(\mathrm{M}+\mathrm{Na})^{+}, 438(\mathrm{M}+\mathrm{H})^{+}$; Anal. Calcd for $\mathrm{C}_{27} \mathrm{H}_{27}$ FeNO: C, 74.15; H, 6.22; N, 3.20. Found: C, 74.02; H, 6.31; N, 3.32.

$\left(R_{\mathrm{p}}\right)$-1-[(4S)-4,5-dihydro-4-tert-butyl-2-oxazolyl]-2-(2-methylphenyl) ferrocene (26f).

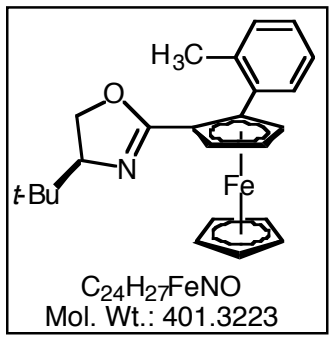

According to the procedure for the preparation of $\mathbf{2 6 a}, O$ tolylboronic acid (126 mg, $0.92 \mathrm{mmol}$ ) and iodide 25 (200 mg, 0.46 mmol) were condensed to afford a red oil which upon purification by column chromatography $\left(\mathrm{CH}_{2} \mathrm{Cl}_{2}\right)$ provided $\mathbf{2 6 f}(163 \mathrm{mg}, 89 \%)$ as an orange foam: $[\square]_{\mathrm{D}}{ }^{26}=+9.7^{\circ},[\square]_{577}{ }^{26}=+10.9^{\circ},[\square]_{546}{ }^{26}=$ $+19.5^{\circ},\left(\mathrm{c}=0.8, \mathrm{CHCl}_{3}\right) ;{ }^{1} \mathrm{H} \mathrm{NMR}\left(400 \mathrm{MHz}, \mathrm{CDCl}_{3}\right) \square 8.07(\mathrm{dd}, J=7.6,1.4 \mathrm{~Hz}, 1 \mathrm{H})$, 7.23 (app dt, $J=7.5,1.9 \mathrm{~Hz}, 2 \mathrm{H}), 7.15-7.17(\mathrm{~m}, 1 \mathrm{H}), 7.07$ (app d, $J=7.5 \mathrm{~Hz}, 1 \mathrm{H}), 4.99$ $(\mathrm{dd}, J=2.6,1.6 \mathrm{~Hz}, 1 \mathrm{H}), 4.19(\mathrm{~s}, 5 \mathrm{H}), 4.09(\mathrm{dd}, J=2.4,1.6 \mathrm{~Hz}, 1 \mathrm{H}), 4.04(\operatorname{app~t}, J=2.5$, 1H), 3.60-3.71 (m, 3H), $2.11(\mathrm{~s}, 3 \mathrm{H}), 0.77(\mathrm{~s}, 9 \mathrm{H}) ;{ }^{13} \mathrm{C}$ NMR (100 MHz, $\left.\mathrm{CDCl}_{3}\right) \square$ 164.7, $138.5,136.8,132.9,129.4,127.2,125.1,92.3,76.6,74.1,71.5,71.0,69.9,68.7,68.0$, 33.7, 26.1, 20.7; IR (film) 1645, $1605 \mathrm{~cm}^{-1}$; LRMS (CI, isobutane) $\mathrm{m} / z, 401(\mathrm{M})^{+}, 344$ (M $-t$-Bu $)^{+}$; Anal. Calcd for $\mathrm{C}_{24} \mathrm{H}_{27}$ FeNO: C, 71.83; H, 6.78; N, 3.49. Found: C, 71.56; H, $6.65 ; \mathrm{N}, 3.29$.

$\left(R_{\mathrm{p}}\right)-1-[(4 S)-4,5-d i h y d r o-4-t e r t-b u t y l-2-o x a z o l y l]-2-(2-m e t h o x y p h e n y l)$ ferrocene

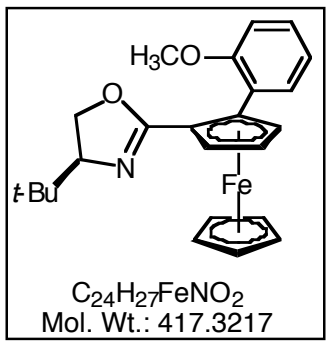

(26g). According to the procedure for the preparation of $\mathbf{2 6 a}, 2$ methoxyphenylboronic acid (148 $\mathrm{mg}, 0.92 \mathrm{mmol}$ ) and iodide 25 (200 $\mathrm{mg}, 0.46 \mathrm{mmol}$ ) were condensed to afford a red oil which upon purification by column chromatography (2:98 acetone/hexanes) provided $\mathbf{2 6 g}(160 \mathrm{mg}, 83 \%)$ as an orange foam: $[\square]_{\mathrm{D}}{ }^{26}=-168.6^{\circ},[\square]_{577}{ }^{26}=-112.8^{\circ},[\square]_{546}{ }^{26}=+28.1^{\circ},\left(c=0.2, \mathrm{CHCl}_{3}\right) ;{ }^{1} \mathrm{H}_{\mathrm{NMR}}(500$ $\left.\left.\mathrm{MHz}^{\mathrm{CDCl}}\right)_{3}\right) \square 8.10(\mathrm{~d}, J=7.4 \mathrm{~Hz}, 1 \mathrm{H}), 7.11-7.14(\mathrm{~m}, 1 \mathrm{H}), 6.69(\mathrm{~d}, J=8.2 \mathrm{~Hz}, 1 \mathrm{H})$, $5.18(\operatorname{app~s,~1H),~} 4.52(\operatorname{app~s,~1H),~} 4.28$ (s, 1H), 4.21 (dd, $J=2.5,2.4 \mathrm{~Hz}, 1 \mathrm{H}), 3.76-3.92$ 
(m, 3H), $3.20(\mathrm{~s}, 3 \mathrm{H}), 1.02(\mathrm{~s}, 9 \mathrm{H}) ;{ }^{13} \mathrm{C} \mathrm{NMR}\left(125 \mathrm{MHz}, \mathrm{C}_{6} \mathrm{D}_{6}\right) \square$ 166.1, 158.9, 134.0, 128.0, 127.2, 120.5, 111.2, 77.2, 74.9, 71.6, 70.9, 69.3, 68.7, 55.7, 34.1, 26.9; IR (film) 3019, 2959, 1643, $1602 \mathrm{~cm}^{-1}$; LRMS (ES) $m / z 440(\mathrm{M}+\mathrm{Na})^{+}, 418(\mathrm{M}+\mathrm{H})^{+}$; Anal. Calcd for $\mathrm{C}_{24} \mathrm{H}_{27} \mathrm{FeNO}_{2}$ : C, 69.07; H, 6.52; N, 3.36. Found: C, 69.25; H, 6.58; N, 3.36.

$\left(R_{\mathrm{p}}\right)-1-[(4 S)-4,5-d i h y d r o-4-t e r t-b u t y l-2-o x a z o l y l]-2-(4-t h i o m e t h y l p h e n y l)$ ferrocene

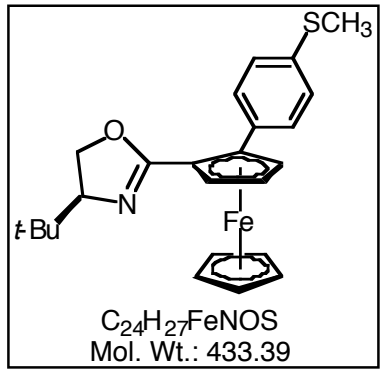

(26h). According to the procedure for the preparation of 26a, 4(methylthio)phenylboronic acid $(672 \mathrm{mg}, 4.0 \mathrm{mmol})$ and iodide 25 (875 mg, $2.0 \mathrm{mmol})$ were condensed to afford a red oil which upon purification by column chromatography $\left(\mathrm{CH}_{2} \mathrm{Cl}_{2}\right)$ provided 26h (660 mg, 76\%) as a red oil which solidified upon standing: $\operatorname{mp~} 86-87^{\circ} \mathrm{C} ;[\square]_{\mathrm{D}}{ }^{26}=-17.0^{\circ},[\square]_{577}{ }^{26}=-19.4^{\circ},[\square]_{546}{ }^{26}=-32.7^{\circ},\left(\mathrm{c}=0.6, \mathrm{CHCl}_{3}\right) ;{ }^{1} \mathrm{H}$ NMR (500 MHz, $\left.\mathrm{CDCl}_{3}\right) \square 7.84(\mathrm{~d}, J=8.3 \mathrm{~Hz}, 1 \mathrm{H}), 7.29(\mathrm{~d}, J=8.3 \mathrm{~Hz}, 2 \mathrm{H}), 5.07$ (app $\mathrm{d}, J=1.7 \mathrm{~Hz}, 1 \mathrm{H}), 4.45(\operatorname{app~d}, J=1.7 \mathrm{~Hz}, 1 \mathrm{H}), 4.20-4.21$ (overlapping peaks, m, 1H, s, 5H), 3.81-3.90 (m, 3H), 2.15 (s, 3H), 0.98 (s, 9H); ${ }^{13} \mathrm{C}$ NMR (100 MHz, $\left.\mathrm{CDCl}_{3}\right) \square$ 164.9, $136.3,134.3,130.4,125.6,88.2,76.4,71.9,71.0,70.9,69.4,69.0,67.9,33.8,26.0,15.9$; IR (film) 2955, 1660, $1599 \mathrm{~cm}^{-1}$; LRMS (ES) $m / z 456(\mathrm{M}+\mathrm{Na})^{+}, 435(\mathrm{M}+\mathrm{H})^{+}, 434(\mathrm{M})^{+}$; Anal. Calcd for $\mathrm{C}_{24} \mathrm{H}_{27}$ FeNOS: C, 66.51; H, 6.28; N, 3.23. Found: C, 66.45; H, 6.45; N, 3.06 .

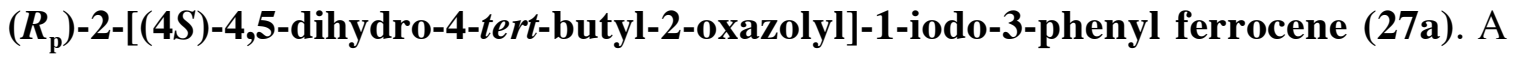

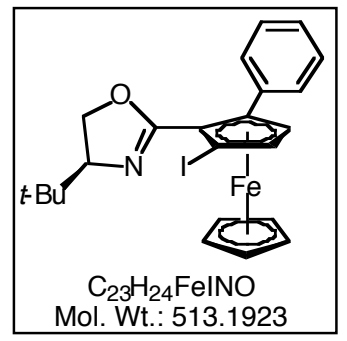
solution of oxazoline $\mathbf{2 6 a}(357 \mathrm{mg}, 0.92 \mathrm{mmol})$ in THF $(9.0 \mathrm{~mL})$ was precooled to $0{ }^{\circ} \mathrm{C}$ and tert-BuLi $(1.5 \mathrm{M}$ in pentane, $0.5 \mathrm{~mL}$, $0.83 \mathrm{mmol})$ was added dropwise. The dark red solution was maintained at $0{ }^{\circ} \mathrm{C}$ for $15 \mathrm{~min}$. A solution of diiodoethane $(233 \mathrm{mg}$, $0.83 \mathrm{mmol})$ in THF $(5.0 \mathrm{~mL})$ was added by cannula. The solution was allowed to warm to room temperature and quenched with $10 \%$ aqueous $\mathrm{Na}_{2} \mathrm{~S}_{2} \mathrm{O}_{3}$ solution $(10 \mathrm{~mL})$. The mixture was extracted with EtOAc $(2 \square 10 \mathrm{~mL})$, the combined EtOAc solution dried $\left(\mathrm{MgSO}_{4}\right)$, and concentrated in vacuo to provide a red oil. 
Purification by column chromatography (5:95 EtOAc/hexanes) provided 27a (332 mg, $78 \%$ based on tert-BuLi) as a light sensitive orange foam: ${ }^{1} \mathrm{H}$ NMR $\left(500 \mathrm{MHz}, \mathrm{CDCl}_{3}\right) \square$ 7.58-7.60 (m, 2H), 7.27-7.34 (m, 3H), $4.70(\mathrm{~d}, J=2.5 \mathrm{~Hz}, 1 \mathrm{H}), 4.64(\mathrm{~d}, J=2.5 \mathrm{~Hz}, 1 \mathrm{H})$, $4.31(\mathrm{dd}, J=10.1,8.5 \mathrm{~Hz}, 1 \mathrm{H}), 4.29(\mathrm{~s}, 5 \mathrm{H}), 4.18(\operatorname{app~t}, J=8.7 \mathrm{~Hz}, 1 \mathrm{H}), 4.08$ (dd, $J=$ 10.1, 8.8 Hz, 1H), 1.02 (s, 9H); ${ }^{13} \mathrm{C}$ NMR (125 MHz, $\left.\mathrm{CDCl}_{3}\right) \square$ 162.6, 136.6, 129.1, 127.7, 126.9, 88.4, 77.04, 76.7, 75.6, 74.2, 70.5, 68.7, 43.4, 33.8, 26.2; IR (film) 2955, 1660, $1602 \mathrm{~cm}^{-1}$; LRMS (ES) $m / z 514(\mathrm{M}+\mathrm{H})^{+}$; HRMS (CI, $\left.\mathrm{NH}_{3}\right) m / z 512.0177$ (512.0174 calcd for $\left.\mathrm{C}_{23} \mathrm{H}_{23} \mathrm{FeINO},(\mathrm{M}-\mathrm{H})^{+}\right)$.

\section{$\left(R_{\mathrm{p}}\right)-2-[(4 S)-4,5-d i h y d r o-4-t e r t-b u t y l-2-o x a z o l y l]-1-i o d o-3-(4-m e t h o x y p h e n y l)$}

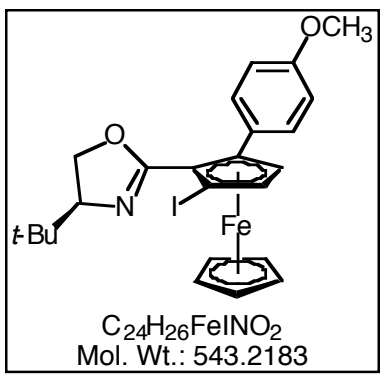

ferrocene (27b). According to the procedure for the preparation of 27a, oxazoline $\mathbf{2 6 \mathbf { b }}(297 \mathrm{mg}, 0.71 \mathrm{mmol})$ in THF $(7.0 \mathrm{~mL})$ was treated with tert-BuLi $(1.40 \mathrm{M}$ in pentane, $0.5 \mathrm{~mL}, 0.70$ mmol) and quenched with a solution of diiodoethane (183 mg, $0.65 \mathrm{mmol})$ in THF (3.5 mL). Purification by column chromatography (10:90 EtOAc/hexanes) provided 27b (259 mg, 73\% based on diiodoethane $)$ as a light sensitive orange foam: $[\square]_{\mathrm{D}}{ }^{26}=+111.0^{\circ}, \quad\left(\mathrm{c}=0.2, \mathrm{CHCl}_{3}\right) ;{ }^{1} \mathrm{H}$ NMR (500 MHz, $\left.\mathrm{CDCl}_{3}\right) \square 7.45$ (app dt, $\left.J=8.8,2.5 \mathrm{~Hz}, 2 \mathrm{H}\right), 6.80$ (app dt, $J=8.8,2.5$ $\mathrm{Hz}, 2 \mathrm{H}), 4.60(\mathrm{~d}, J=2.5 \mathrm{~Hz}, 1 \mathrm{H}), 4.52(\mathrm{~d}, J=2.5 \mathrm{~Hz}, 1 \mathrm{H}), 4.27(\mathrm{dd}, J=10.1,8.6 \mathrm{~Hz}$, 1H), $4.21(\mathrm{~s}, 5 \mathrm{H}), 4.11(\operatorname{app~t}, J=8.6 \mathrm{~Hz}, 1 \mathrm{H}), 4.00(\mathrm{dd}, J=10.1,8.8 \mathrm{~Hz}, 1 \mathrm{H}), 3.80(\mathrm{~s}$, 3H), 0.95 (s, 9H); ${ }^{13} \mathrm{C}$ NMR (125 MHz, $\left.\mathrm{CDCl}_{3}\right) \square 162.7,158.6,130.1,128.6,113.2,88.4$, 76.9, 76.2, 75.3, 74.0, 70.2, 68.6, 55.2, 43.3, 33.7, 26.2; IR (film) 2956, 1661, $1612 \mathrm{~cm}^{-1}$; LRMS (ES) $m / z 566(\mathrm{M}+\mathrm{Na})^{+}, 544(\mathrm{M}+\mathrm{H})^{+} ; \mathrm{HRMS}\left(\mathrm{CI}, \mathrm{NH}_{3}\right) m / z 543.0358(543.0360$ calcd for $\mathrm{C}_{24} \mathrm{H}_{26} \mathrm{FeINO}_{2}, \mathrm{M}^{+}$). 


\section{$\left(R_{\mathrm{p}}\right)$-2-[(4S)-4,5-dihydro-4-tert-butyl-2-oxazolyl]-1-iodo-3-(4-trifluoromethylphenyl)}

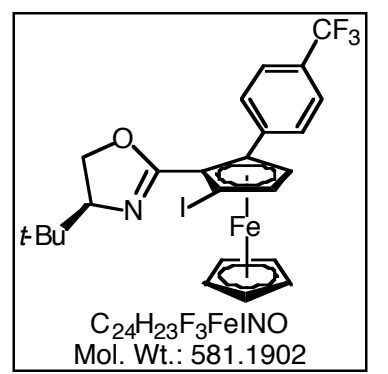

ferrocene (27c). According to the procedure for the preparation of 27a, oxazoline $\mathbf{2 6 c}(100 \mathrm{mg}, 0.22 \mathrm{mmol})$ in THF $(2.0 \mathrm{~mL})$ was treated with tert-BuLi (1.25 M in pentane, $0.16 \mathrm{~mL}, 0.2 \mathrm{mmol})$ and quenched with a solution of diiodoethane $(60 \mathrm{mg}, 0.21 \mathrm{mmol})$ in THF (1.0 mL). Purification by column chromatography $\left(\mathrm{CH}_{2} \mathrm{Cl}_{2}\right)$ provided $27 \mathrm{c}(66 \mathrm{mg}, 57 \%$ based on tert-BuLi) as a light sensitive orange foam: $[\square]_{\mathrm{D}}{ }^{26}=-56.0^{\circ},\left(\mathrm{c}=0.1, \mathrm{CHCl}_{3}\right) ;{ }^{1} \mathrm{H} \mathrm{NMR}\left(500 \mathrm{MHz}, \mathrm{CDCl}_{3}\right) \square 7.45(\mathrm{~d}, J=8.1 \mathrm{~Hz}, 2 \mathrm{H})$, $7.50(\mathrm{~d}, J=8.2 \mathrm{~Hz}, 2 \mathrm{H}), 4.69(\mathrm{~d}, J=2.5 \mathrm{~Hz}, 1 \mathrm{H}), 4.61(\mathrm{~d}, J=2.5 \mathrm{~Hz}, 1 \mathrm{H}), 4.30(\mathrm{dd}, J=$ 10.0, 8.7 Hz, 1H), $4.23(\mathrm{~s}, 5 \mathrm{H}), 4.12(\operatorname{app~t}, J=8.7 \mathrm{~Hz}, 1 \mathrm{H}), 4.02(\operatorname{app~t}, J=9.5 \mathrm{~Hz}, 1 \mathrm{H})$, $0.95(\mathrm{~s}, 9 \mathrm{H}) ;{ }^{13} \mathrm{C} \mathrm{NMR}\left(125 \mathrm{MHz}, \mathrm{CDCl}_{3}\right) \square 162.3,141.1,129.2,128.8(\mathrm{q}, J=32.3 \mathrm{~Hz})$, $124.6(\mathrm{q}, J=3.6 \mathrm{~Hz}), 124.3(\mathrm{q}, J=270.1 \mathrm{~Hz}), 86.5,77.04,76.9,76.2,74.3,70.8,68.7$, 43.3, 33.8, 26.2; IR (film) 2955, 1658, $1617 \mathrm{~cm}^{-1}$; LRMS (ES) $m / z 604(\mathrm{M}+\mathrm{Na})^{+}, 582$ $(\mathrm{M}+\mathrm{H})^{+}$; HRMS (EI) $m / z 581.0117$ (581.0127 calcd for $\mathrm{C}_{24} \mathrm{H}_{23} \mathrm{~F}_{3} \mathrm{FeINO}, \mathrm{M}^{+}$).

\section{$\left(R_{\mathrm{p}}\right)-2-[(4 S)-4,5-d i h y d r o-4-t e r t-b u t y l-2-o x a z o l y l]-1-i o d o-3-(1-n a p t h y l)$ ferrocene}

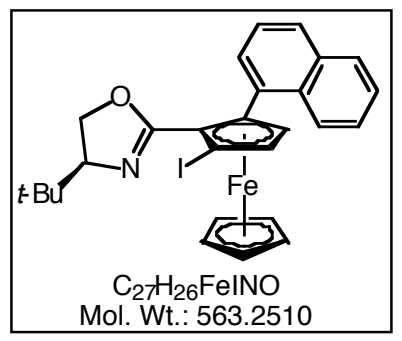
(27e). According to the procedure for the preparation of $27 \mathbf{a}$, oxazoline 26e (400 $\mathrm{mg}, 0.91 \mathrm{mmol})$ in THF $(9.0 \mathrm{~mL})$ was treated with tert-BuLi (1.25 $\mathrm{M}$ in pentane, $0.7 \mathrm{~mL}, 0.87 \mathrm{mmol})$ and quenched with diiodoethane $(268 \mathrm{mg}, 0.87 \mathrm{mmol})$ in THF (4.5 mL). Purification by column chromatography $(2: 98$

EtOAc/hexanes) provided 27e (323 mg, 66\% based on tert-BuLi) as a light sensitive orange foam: $[\square]_{\mathrm{D}}{ }^{26}=+20.0^{\circ},[\square]_{577}{ }^{26}=+23.8^{\circ},[\square]_{546}{ }^{26}=+23.8^{\circ},[\square]_{435}{ }^{26}=+89.2^{\circ},[\square]_{405}{ }^{26}$ $=+22.5^{\circ},\left(\mathrm{c}=0.2, \mathrm{CHCl}_{3}\right) ;{ }^{1} \mathrm{H} \mathrm{NMR}\left(500 \mathrm{MHz}, \mathrm{CDCl}_{3}\right) \square 7.98(\mathrm{~d}, J=7.0 \mathrm{~Hz}, 1 \mathrm{H})$, 7.76-7.81 (m, 3H), $7.56(\operatorname{app~t}, J=7.9 \mathrm{~Hz}, 1 \mathrm{H}), 7.39$ (app t, $J=7.4 \mathrm{~Hz}, 1 \mathrm{H}), 7.34$ (app t, $\mathrm{J}=7.5 \mathrm{~Hz}, 1 \mathrm{H}), 4.77(\mathrm{~d}, J=2.4 \mathrm{~Hz}, 1 \mathrm{H}), 4.78(\mathrm{~d}, J=2.6 \mathrm{~Hz}, 1 \mathrm{H}), 4.37$ (s, 5H), 4.01 (app $\mathrm{t}, J=12.6 \mathrm{~Hz}, 1 \mathrm{H}), 3.73-3.77(\mathrm{~m}, 2 \mathrm{H}), 0.87(\mathrm{~s}, 9 \mathrm{H}) ;{ }^{13} \mathrm{C} \mathrm{NMR}\left(125 \mathrm{MHz}, \mathrm{CDCl}_{3}\right) \square$ $162.3,133.3,133.2,133.1,129.3,128.0,127.7,125.9,125.8,125.4,124.7,90.0,76.7$, 76.5, 75.7, 74.1, 73.8, 68.2, 42.0, 33.5, 25.8; IR (film) 3049, 2954, 1657, $1594 \mathrm{~cm}^{-1}$; 
LRMS (ES) $m / z 586(\mathrm{M}+\mathrm{Na})^{+}, 564(\mathrm{M}+\mathrm{H})^{+} ;$HRMS (EI) $m / z 563.0423(563.0410$ calcd for $\mathrm{C}_{27} \mathrm{H}_{26} \mathrm{FeINO}, \mathrm{M}^{+}$).

\section{$\left(R_{\mathrm{p}}\right)$-2-[(4S)-4,5-dihydro-4-tert-butyl-2-oxazolyl]-1-iodo-3-(2-methylphenyl)}

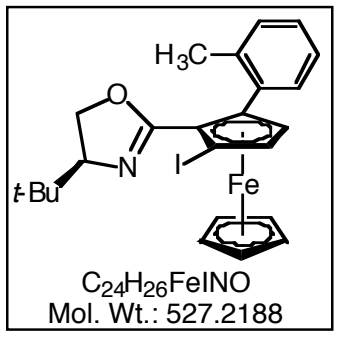

ferrocene (27f). According to the procedure for the preparation of 27a, oxazoline $26 \mathbf{f}(410 \mathrm{mg}, 1.02 \mathrm{mmol})$ in THF $(10.0 \mathrm{~mL})$ and tertBuLi (1.5 M in pentane, $0.6 \mathrm{~mL}, 0.92 \mathrm{mmol})$ was quenched with a solution of diiodoethane $(259 \mathrm{mg}, 0.92 \mathrm{mmol})$ in THF $(5.0 \mathrm{~mL})$. Purification by column chromatography (5:95 EtOAc/hexanes) provided $27 f\left(361 \mathrm{mg}, 74 \%\right.$ based on tert-BuLi) as a light sensitive orange foam: $[\square]_{\mathrm{D}}{ }^{26}=$ $+45.0^{\circ},[\square]_{577}{ }^{26}=+33.2^{\circ},[\square]_{546}{ }^{26}=+23.2^{\circ},\left(\mathrm{c}=0.2, \mathrm{CHCl}_{3}\right) ;{ }^{1} \mathrm{H} \mathrm{NMR}\left(500 \mathrm{MHz}, \mathrm{CDCl}_{3}\right)$ $\square 7.79$ (app dd, $J=8.9,2.7 \mathrm{~Hz}, 1 \mathrm{H}), 7.14-7.24$ (m, 2H), 7.09-7.11(m, 1H), 4.66 (d, $J=$ $2.4 \mathrm{~Hz}, 1 \mathrm{H}), 4.37(\mathrm{~d}, J=2.4 \mathrm{~Hz}, 1 \mathrm{H}), 4.33(\mathrm{~s}, 5 \mathrm{H}), 4.12(\mathrm{dd}, J=10.0,8.7 \mathrm{~Hz}, 1 \mathrm{H}), 4.00$ (app t, $J=8.2 \mathrm{~Hz}, 1 \mathrm{H}), 3.82(\mathrm{dd}, J=10.1,7.8 \mathrm{~Hz}, 1 \mathrm{H}), 2.08(\mathrm{~s}, 3 \mathrm{H}), 0.77(\mathrm{~s}, 9 \mathrm{H}) ;{ }^{13} \mathrm{C}$ NMR (125 MHz, $\left.\mathrm{CDCl}_{3}\right) \square 162.3,138.2,134.7,132.1,129.2,127.1,124.7,91.3,76.5$, 76.4, 75.3, 73.9, 72.6, 68.2, 42.2, 33.6, 25.8, 20.6; IR (film) 2952, 1651, $1603 \mathrm{~cm}^{-1}$; LRMS (ES) $m / z 550(\mathrm{M}+\mathrm{Na})^{+}, 528(\mathrm{M}+\mathrm{H})^{+}$; HRMS (CI, $\left.\mathrm{NH}_{3}\right) m / z 527.0407$ (527.0409 calcd for $\mathrm{C}_{24} \mathrm{H}_{26} \mathrm{FeINO}, \mathrm{M}^{+}$).

\section{$\left(R_{\mathrm{p}}\right)$-2-[(4S)-4,5-dihydro-4-tert-butyl-2-oxazolyl]-1-iodo-3-(4-methylthiophenyl)}

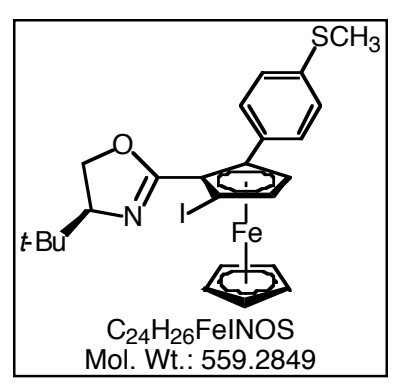

ferrocene (27h). According to the procedure for the preparation of $27 \mathbf{a}$, oxazoline $26 \mathbf{h}(660 \mathrm{mg}, 1.52 \mathrm{mmol})$ in THF $(15.0 \mathrm{~mL})$ and tert-BuLi (0.77 $\mathrm{M}$ in pentane, $1.9 \mathrm{~mL}, 1.44 \mathrm{mmol})$ was quenched with a solution of diiodoethane (406 $\mathrm{mg}, 1.44 \mathrm{mmol})$ in THF (7.5 mL). Purification by column chromatography $\left(\mathrm{CH}_{2} \mathrm{Cl}_{2}\right)$ provided $27 \mathbf{h}(644 \mathrm{mg}, 80 \%$ based on tert-BuLi) as a light sensitive orange foam: []$_{\mathrm{D}}{ }^{26}=+120.0,\left(\mathrm{c}=0.1, \mathrm{CHCl}_{3}\right) ;{ }^{1} \mathrm{H} \mathrm{NMR}(400 \mathrm{MHz}$, $\left.\mathrm{CDCl}_{3}\right) \square 7.48($ app dt, $J=8.6,2.0 \mathrm{~Hz}, 2 \mathrm{H}), 7.18(\operatorname{app~dt}, J=8.5,2.0 \mathrm{~Hz}, 2 \mathrm{H}), 4.67(\mathrm{~d}, J$ $=2.5 \mathrm{~Hz}, 1 \mathrm{H}), 4.59(\mathrm{~d}, J=2.5 \mathrm{~Hz}, 1 \mathrm{H}), 4.32(\mathrm{dd}, J=10.1,8.5 \mathrm{~Hz}, 1 \mathrm{H}), 4.25(\mathrm{~s}, 5 \mathrm{H}), 4.12$ 
(app t, $J=8.6 \mathrm{~Hz}, 1 \mathrm{H}), 4.05(\mathrm{dd}, J=10.1,8.8 \mathrm{~Hz}, 1 \mathrm{H}), 2.52(\mathrm{~s}, 3 \mathrm{H}), 1.00(\mathrm{~s}, 9 \mathrm{H}) ;{ }^{13} \mathrm{C}$ NMR (125 MHz, $\left.\mathrm{CDCl}_{3}\right) \square 162.5,136.9,133.5,129.4,126.0,87.9,77.3,76.6,75.9,74.4$, 70.6, 69.0, 43.8, 34.2, 26.7, 16.3; IR (film) 2953, 1657, $1599 \mathrm{~cm}^{-1}$; LRMS (ES) $\mathrm{m} / z$. 582 $(\mathrm{M}+\mathrm{Na})^{+}, 560(\mathrm{M}+\mathrm{H})^{+} ;$HRMS (EI) $m / z 559.0130$ (559.0131 calcd for $\mathrm{C}_{24} \mathrm{H}_{26}$ FeINOS, $\left.\mathrm{M}^{+}\right)$.

$\left(\boldsymbol{R}_{\mathrm{p}}\right)$-bis- $\square$-iodo-\{2-[(4S)-2-(4,5-dihydro-4-tert-butyl-2-oxazolyl)]-3-phenyl-ferrocenyl $\}$

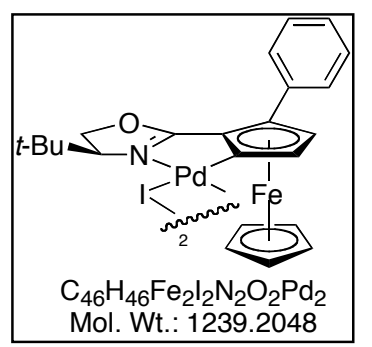
dipalladium (29). To a solution of iodide $\mathbf{2 7 a}$ (361 mg, 0.69 mmol) in benzene $(15.0 \mathrm{~mL}), \mathrm{Pd}_{2} \mathrm{dba}_{3} \cdot \mathrm{CHCl}_{3}(427 \mathrm{mg}, 0.41$ mmol) was added in one portion. The dark red solution was protected from light and maintained for $18 \mathrm{~h}$ before filtering through a plug of Florisil (100-200 mesh, benzene), and concentrating in vacuo to provide a red solid. Purification of the residue by column chromatography (1:2 hexanes/benzene) provided $29(350 \mathrm{mg}, 81 \%)$ as a mixture of isomers, red prisms.: mp 190-194 ${ }^{\circ} \mathrm{C} ;{ }^{1} \mathrm{H}$ NMR (400 $\left.\mathrm{MHz}, \mathrm{CDCl}_{3}\right) \square 7.49-7.52(\mathrm{~m}, 4 \mathrm{H})$, 7.21-7.29 (m, 6H $)^{20}, 5.03-5.07(\mathrm{~m}, 2 \mathrm{H}), 4.75-4.78(\mathrm{~m}, 2 \mathrm{H}), 4.39-4.43(\mathrm{~m}, 10 \mathrm{H})$, 3.92-3.95 (m, 5H), $1.03(\mathrm{~s}, 9 \mathrm{H}), 1.02(\mathrm{~s}, 9 \mathrm{H}) ;{ }^{13} \mathrm{C}$ NMR (125 MHz, $\left.\mathrm{CDCl}_{3}\right) \square$ 179.9, $179.5,136.8,129.1,128.8,128.0,127.9,126.8,99.7,99.2,86.7,79.1,78.5,74.6,73.2$, $73.1,73.0,72.8,71.8,71.6,71.2,70.1,70.0,69.5,35.8,35.8,30.1,27.2,27.1$, (not all carbon signals for the two isomers are resolved); IR (film) $2961,1583 \mathrm{~cm}^{-1}$; MS (FAB, MNBA) $m / z$ 1239, 619, 388; Anal. Calcd for $\mathrm{C}_{46} \mathrm{H}_{48} \mathrm{Fe}_{2} \mathrm{I}_{2} \mathrm{~N}_{2} \mathrm{O}_{2} \mathrm{Pd}_{2}$ : C, 44.58; H, 3.90; N, 2.26. Found: C, 44.39; H, 3.78; N, 2.24.

\section{$\left(\boldsymbol{R}_{\mathrm{p}}\right)$-bis- $\square$-iodo-\{2-[(4S)-2-(4,5-dihydro-4-tert-butyl-2-oxazolyl)]-3-(4-}

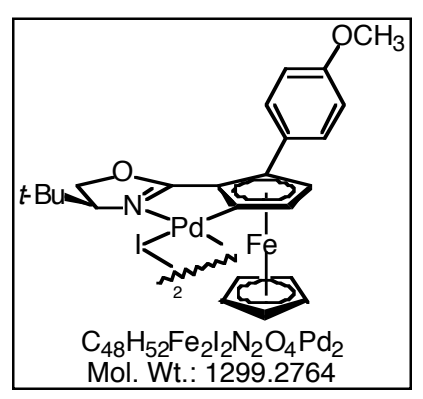

methoxyphenyl)-ferrocenyl \} dipalladium (30). A solution of iodide $27 \mathbf{b}(241 \mathrm{mg}, 0.44 \mathrm{mmol})$ in benzene $(17.0 \mathrm{~mL})$ was allowed to react with $\mathrm{Pd}_{2} \mathrm{dba}_{3} \cdot \mathrm{CHCl}_{3}(280 \mathrm{mg}, 0.27 \mathrm{mmol})$ as described for the preparation of 29. Purification of the crude product by column chromatography (1:2 hexanes/benzene) 
provided 30 (272 $\mathrm{mg}, 95 \%)$. Filtration through a plug of $\mathrm{SiO}_{2}\left(\mathrm{CH}_{2} \mathrm{Cl}_{2}\right)$ provided analytically pure $\mathbf{3 0}$ as a mixture of isomers, red prisms (257 mg, 90\%): $\mathrm{mp} 201{ }^{\circ} \mathrm{C}$; $[\square]_{\mathrm{D}}{ }^{26}=+20.5^{\circ},[\square]_{577}{ }^{26}=+23.8^{\circ},[\square]_{546}{ }^{26}=+33.2^{\circ},\left(\mathrm{c}=0.2, \mathrm{CHCl}_{3}\right) ;{ }^{1} \mathrm{H}$ NMR $(500$ $\left.\mathrm{MHz}, \mathrm{CDCl}_{3}\right) \square 7.43-7.45(\mathrm{~m}, 4 \mathrm{H}), 6.80-6.83(\mathrm{~m}, 4 \mathrm{H}), 5.01-5.04(\mathrm{~m}, 2 \mathrm{H}), 4.72-4.77(\mathrm{~m}$, 2H), 4.54-4.58 (m, 2H), 4.42 (s, 5H), 4.38 (s, 5H), 3.91-3.95 (m, 2H), $3.80(\mathrm{~m}, 6 \mathrm{H}), 1.02$ (s, 18H); ${ }^{13} \mathrm{C}$ NMR $\left(125 \mathrm{MHz}, \mathrm{CDCl}_{3}\right) \square 179.7,179.3,158.2,129.6,128.5,129.6,128.5$, 113.3, 113.2, 99.4, 98.9, 86.5, 78.6, 77.9, 74.3, 72.8, 72.7, 70.9, 70.8, 69.7, 69.1, 55.3, 35.5, 26.9, 26.8, (not all carbon signals for the isomers are resolved); IR (film) 2961, $1586 \mathrm{~cm}^{-1}$; MS (MALDI) $\mathrm{m} / \mathrm{z} 1340,1152,978,857,669$; Anal. Calcd for $\mathrm{C}_{48} \mathrm{H}_{52} \mathrm{Fe}_{2} \mathrm{I}_{2} \mathrm{~N}_{2} \mathrm{O}_{4} \mathrm{Pd}_{2}$ : C, 44.37; H, 4.03; N, 2.16. Found: C, 44.59; H, 4.18; N, 2.10 .

\section{$\left(R_{\mathrm{p}}\right)$-bis-प-iodo-\{2-[(4S)-2-(4,5-dihydro-4-tert-butyl-2-oxazolyl)]-3-(4-}

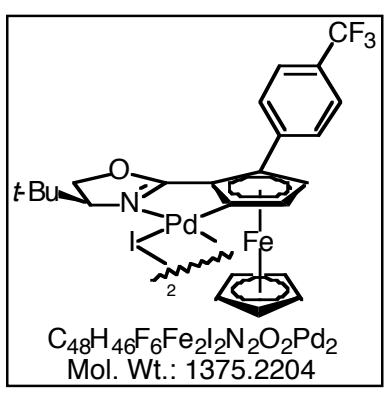

trifluoromethylphenyl)-ferrocenyl\} dipalladium (31). A solution of iodide $27 \mathrm{c}(113 \mathrm{mg}, 0.19 \mathrm{mmol})$ in benzene $(4.0 \mathrm{~mL})$ was allowed to react with $\mathrm{Pd}_{2} \mathrm{dba}_{3} \cdot \mathrm{CHCl}_{3}(118 \mathrm{mg}, 0.11 \mathrm{mmol})$ as described for the preparation of 29. Purification of the crude product by column chromatography (1:1 hexanes/benzene) provided 31a (116 mg, 89\%) as a mixture of isomers, red prisms: $\operatorname{mp} 220{ }^{\circ} \mathrm{C} ;[\square]_{\mathrm{D}}{ }^{26}=+172.9^{\circ},[\square]_{577}{ }^{26}=+137.7^{\circ},[\square]_{546}{ }^{26}=+43.2^{\circ}, \quad\left(\mathrm{c}=0.4, \mathrm{CHCl}_{3}\right) ;{ }^{1} \mathrm{H}$ NMR (500 MHz, $\left.\mathrm{CDCl}_{3}\right) \square 7.61-7.63(\mathrm{~m}, 4 \mathrm{H}), 7.49-7.52(\mathrm{~m}, 4 \mathrm{H}), 5.12(\mathrm{~d}, J=2.3 \mathrm{~Hz}$, 1H), 5.09 (d, $J=2.2 \mathrm{~Hz}, 1 \mathrm{H}), 4.78$ (app td, $J=9.4,2.3 \mathrm{~Hz}, 2 \mathrm{H}), 4.68-4.67$ (m, 2H), 4.55-4.61 (m, 2H), $4.43(\mathrm{~s}, 5 \mathrm{H}), 4.40(\mathrm{~s}, 5 \mathrm{H}), 3.93-3.97(\mathrm{~m}, 2 \mathrm{H}), 0.994(\mathrm{~s}, 9 \mathrm{H}), 0.991(\mathrm{~s}$, $9 \mathrm{H}) ;{ }^{13} \mathrm{C}$ NMR $\left(125 \mathrm{MHz}, \mathrm{CDCl}_{3}\right) \square 179.8,179.4,141.3,128.9,128.5$ (q, $\left.J=32.3 \mathrm{~Hz}\right)$, $124.8(\mathrm{q}, J=3.5 \mathrm{~Hz}), 124.3(\mathrm{q}, J=270.3), 99.6,99.1,84.3,79.5,78.8,74.42,74.38$, 73.1, 72.9, 71.8, 71.6, 71.0, 70.1, 69.9, 69.5, 35.4, 26.7, 26.6 (not all carbon signals for the two isomers are resolved); IR (film) 2961, 1617, $1583 \mathrm{~cm}^{-1}$; MS (FAB, MNBA) $\mathrm{m} / z$ $1376,1247,793,687,560,154$. 


\section{$\left(R_{\mathrm{p}}\right)$-bis- $\square$-iodo-\{2-[(4S)-2-(4,5-dihydro-4-tert-butyl-2-oxazolyl)]-3-(2,6-}

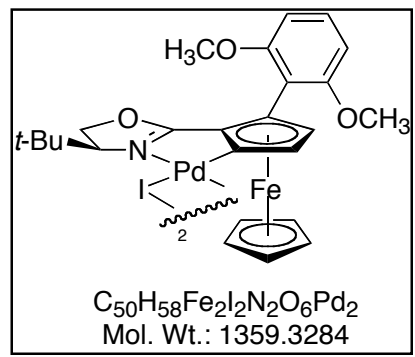

dimethoxyphenyl)-ferrocenyl\} dipalladium (32). According to the procedure for the preparation of iodide $\mathbf{2 7} \mathbf{a}$, oxazoline 26d $(250 \mathrm{mg}, 0.56 \mathrm{mmol})$ in THF $(7.0 \mathrm{~mL})$ was treated with tert-BuLi (1.25 $\mathrm{M}$ in pentane, $0.42 \mathrm{~mL}, 0.53 \mathrm{mmol})$ and quenched with a solution of diiodoethane $(150 \mathrm{mg}, 0.53 \mathrm{mmol})$

in THF (7.0 mL). Purification by column chromatography $\left(\mathrm{CH}_{2} \mathrm{Cl}_{2}\right)$ provided crude $27 \mathbf{d}$ (173 mg, 61\% based on tert-BuLi) as a light sensitive orange foam which was carried on directly: LRMS (ES) $m / z, 534(\mathrm{M}+\mathrm{H})^{+}$.

A solution of crude iodide $27 \mathbf{d}(190 \mathrm{mg}, 0.33 \mathrm{mmol})$ in benzene $(10.0 \mathrm{~mL})$ was allowed to react with $\mathrm{Pd}_{2} \mathrm{dba}_{3} \cdot \mathrm{CHCl}_{3}(207 \mathrm{mg}, 0.20 \mathrm{mmol})$ as described for the preparation of 29. Purification of the crude product by column chromatography $(1: 2$ hexanes/benzene) provided $32(212 \mathrm{mg}, 95 \%)$. Filtration through a plug of $\mathrm{SiO}_{2}\left(\mathrm{CH}_{2} \mathrm{Cl}_{2}\right)$ provided analytically pure 32 as a mixture of isomers, red prisms (202 mg, 90\%): mp 223 ${ }^{\circ} \mathrm{C} ;[\square]_{\mathrm{D}}{ }^{26}=+119.9^{\circ},[\square]_{577}{ }^{26}=+127.5^{\circ},[\square]_{546}{ }^{26}=+126.9^{\circ},[\square]_{435}{ }^{26}=-108.3^{\circ},[\square]_{405}{ }^{26}=$ $-207.3^{\circ},\left(\mathrm{c}=0.3, \mathrm{CHCl}_{3}\right) ;{ }^{1} \mathrm{H}$ NMR $\left(400 \mathrm{MHz}, \mathrm{CDCl}_{3}\right) \square 7.20(\mathrm{~m}, 2 \mathrm{H}), 6.57-6.61(\mathrm{~m}$, 2H), $6.48(\mathrm{~d}, J=8.3 \mathrm{~Hz}, 2 \mathrm{H}), 5.15(\operatorname{app~t}, J=2.5 \mathrm{~Hz}, 2 \mathrm{H}), 4.59-4.16$ (m, 2H), 4.44-4.49 (m, 14H), 3.86-3.91 (m, 6H), 3.51 (br s, 6H), 1.05 (s, 9H), 1.03 (s, 9H); ${ }^{13} \mathrm{C} \mathrm{NMR} \mathrm{(100}$ $\left.\mathrm{MHz}, \mathrm{CDCl}_{3}\right) \square 180.8,180.4,158.5,157.5,128.0,112.2$, 112.1, 103.7, 103.5, 98.3, 97.9, 78.0, 77.6, 77.24, 77.20, 77.16, 74.0, 73.2, 72.7, 72.6, 71.9, 71.7, 70.6, 69.4, 56.0, 55.8, 55.3, 35.1, 35.0, 26.93, 26.90 (not all carbon signals for the two isomers are resolved); IR (film) 2832, $1585 \mathrm{~cm}^{-1}$; MS (MALDI) $\mathrm{m} / \mathrm{z}$ 1360, 1172, 893, 679; Anal. Calcd for $\mathrm{C}_{50} \mathrm{H}_{58} \mathrm{Fe}_{2} \mathrm{I}_{2} \mathrm{~N}_{2} \mathrm{O}_{6} \mathrm{Pd}_{2}:$ C, 44.18; H, 4.15; N, 2.06. Found: C, 44.08; H, 4.16; N, 2.05.

\section{$\left(R_{\mathrm{p}}\right)$-bis- $\square$-iodo-\{2-[(4S)-2-(4,5-dihydro-4-tert-butyl-2-oxazolyl)]-3-(1-napthyl)-}

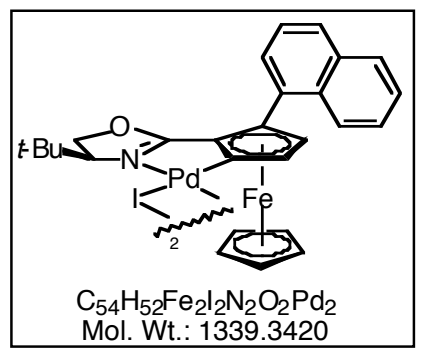

ferrocenyl\} dipalladium (33). According to the procedure for the preparation of palladacycle 29 , iodide 27 e $(260 \mathrm{mg}, 0.46$ $\mathrm{mmol})$ in benzene $(17.0 \mathrm{~mL})$ was reacted with $\mathrm{Pd}_{2} \mathrm{dba}_{3} \cdot \mathrm{CHCl}_{3}$ (287 $\mathrm{mg}, 0.28 \mathrm{mmol}$ ). Purification of the residue by column 
chromatography (1:2 hexanes/benzene) provided 33 (305 mg, 99\%). Filtration through a plug of silica $\left(\mathrm{CH}_{2} \mathrm{Cl}_{2}\right)$ provided analytically pure 33 as a mixture of isomers, red prisms (298 mg, 98\%): $\mathrm{mp} 210^{\circ} \mathrm{C} ;[\square]_{\mathrm{D}}{ }^{26}=+20.0^{\circ},[\square]_{577}{ }^{26}=+23.8^{\circ},[\square]_{546}{ }^{26}=+23.8^{\circ},[\square]_{435}{ }^{26}=$ $+89.2^{\circ},[\square]_{405}{ }^{26}=+22.5^{\circ},\left(\mathrm{c}=0.2, \mathrm{CHCl}_{3}\right) ;{ }^{1} \mathrm{H} \mathrm{NMR}\left(500 \mathrm{MHz}, \mathrm{CDCl}_{3}\right) \square 7.96$ (app t, $J$ $=6.8 \mathrm{~Hz}, 2 \mathrm{H}$ ), 7.77-7.85 (overlapping $\mathrm{m}, 6 \mathrm{H}$ ), 7.45-7.50 (m, 2H), 7.40 (app t, $J=7.5$ $\mathrm{Hz}, 2 \mathrm{H}$ ), 7.27 (app t, J = 7.4, 2H), 5.13-5.15 (m, 2H), 4.58-4.61 (overlapping m, 12H), 4.46 (app d, $J=9.0 \mathrm{~Hz}, 2 \mathrm{H}), 4.37$ (app t, $J=8.9 \mathrm{~Hz}, 2 \mathrm{H}), 3.87-3.89$ (m, 2H), 0.994 (s, 9H), 0.991 (s, 9H); ${ }^{13} \mathrm{C}$ NMR (125 MHz, $\left.\mathrm{CDCl}_{3}\right) \square$ 179.5, 179.1, 132.99, 132.96, 132.91, 129.0, 128.9, 127.9, 127.4, 125.8, 125.41, 125.39, 124.8, 124.7, 98.0, 97.5, 86.7, 86.6, $77.8,77.1,74.4,74.3,74.2,74.1,72.9,72.8,71.8,70.8,69.6,35.40,35.35,26.92,26.87$ (not all carbon signals for the two isomers are resolved); IR (film) 2961, $1586 \mathrm{~cm}^{-1}$; MS (MALDI) $m / z$ 1340, 1152, 978, 857, 669; Anal. Calcd for $\mathrm{C}_{54} \mathrm{H}_{52} \mathrm{Fe}_{2} \mathrm{I}_{2} \mathrm{~N}_{2} \mathrm{O}_{2} \mathrm{Pd}_{2}$ : C, 48.43; H, 3.91; N, 2.16. Found: C, 48.59; H, 4.08; N, 2.10.

\section{$\left(R_{\mathrm{p}}\right)$-bis-प-iodo-\{2-[(4S)-2-(4,5-dihydro-4-tert-butyl-2-oxazolyl)]-3-(2-methylphenyl)-}

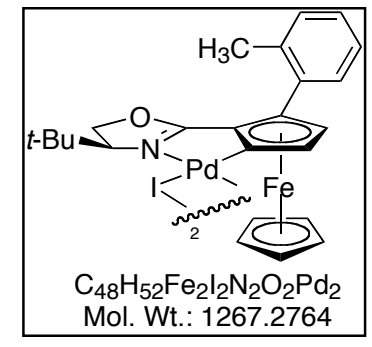

ferrocenyl) dipalladium (34). A solution of iodide $27 f(361 \mathrm{mg}$, $0.69 \mathrm{mmol})$ in benzene $(15.0 \mathrm{~mL})$ was allowed to react with $\mathrm{Pd}_{2} \mathrm{dba}_{3} \cdot \mathrm{CHCl}_{3}(427 \mathrm{mg}, 0.41 \mathrm{mmol}$ ) as described for the preparation of 29. Purification of the crude product by column chromatography (1:2 hexanes/benzene) provided 34 (350 mg, $81 \%$ ) as a mixture of isomers, red prisms: $\mathrm{mp} 180-181{ }^{\circ} \mathrm{C}$; [D] $]_{\mathrm{D}}{ }^{26}=+160.9^{\circ},[\square]_{577}{ }^{26}=$ $+147.7^{\circ},[\square]_{546}{ }^{26}=+113.2^{\circ},\left(\mathrm{c}=0.4, \mathrm{CHCl}_{3}\right) ;{ }^{1} \mathrm{H}$ NMR $\left(500 \mathrm{MHz}, \mathrm{CDCl}_{3}\right) \square 7.67-7.71$ (m, 2H), 7.06-7.13 (m, 4H), 6.98-6.99 (m, 2H), 4.93-4.95 (m, 2H), 4.50-4.52 (m, 2H), 4.47 (s, 5H), 4.43 (s, 5H), 4.35 (app t, $J=9.0 \mathrm{~Hz}, 2 \mathrm{H}), 4.42-4.26$ (m, 2H), 3.77-3.81 (m, 2H), 1.95 (s, 6H), 0.91 (s, 18H); ${ }^{13} \mathrm{C}$ NMR (125 MHz, $\left.\mathrm{CDCl}_{3}\right) \square$ 180.0, 137.7, 135.2, 132.6, 132.5, 130.0, 128.8, 127.5, 125.5, 125.4, 98.6, 98.1, 88.7, 88.6, 78.0, 74.7, 74.6, $73.8,73.7,73.2,73.1,71.7,71.2,70.0,35.7,35.6,27.24,27.18,20.7$ (not all carbon signals for the two isomers are resolved); IR (film) 2961, 1617, $1583 \mathrm{~cm}^{-1}$; MS (FAB, 
MNBA) $m / z$ 1268, 154; Anal. Calcd for $\mathrm{C}_{48} \mathrm{H}_{52} \mathrm{Fe}_{2} \mathrm{I}_{2} \mathrm{~N}_{2} \mathrm{O}_{2} \mathrm{Pd}_{2}: \mathrm{C}$, 45.49; $\mathrm{H}, 4.14 ; \mathrm{N}, 2.21$. Found: C, 45.39; H, 4.10; N, 1.90.

$\left(R_{\mathrm{p}}\right)$-bis- $\square$-iodo-\{2-[(4S)-2-(4,5-dihydro-4-tert-butyl-2-oxazolyl)]-3-(2methoxyphenyl)-ferrocenyl\} dipalladium (35). According to the procedure for the

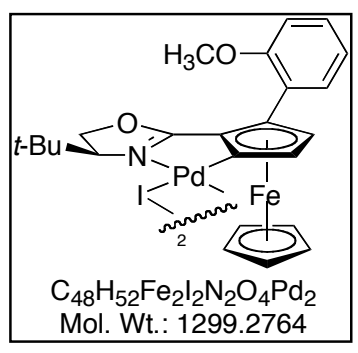
preparation of iodide $\mathbf{2 7 a}$, oxazoline $\mathbf{2 6 g}$ (290 $\mathrm{mg}, 0.69 \mathrm{mmol})$ in THF $(2.5 \mathrm{~mL})$ was treated with tert-BuLi $(1.40 \mathrm{M}$ in pentane, $0.48 \mathrm{~mL}, 0.67 \mathrm{mmol}$ ) and quenched with a solution of diiodoethane (184 mg, $0.67 \mathrm{mmol})$ in THF $(2.0 \mathrm{~mL})$. Purification by column chromatography $\left(\mathrm{CH}_{2} \mathrm{Cl}_{2}\right)$ provided crude $\mathbf{2 7 g}$ (321 $\mathrm{mg}, 80 \%$ based on tert-BuLi) as a light sensitive orange foam: LRMS (ES) $m / z 566(\mathrm{M}+$ $\mathrm{Na})^{+}, 544(\mathrm{M}+\mathrm{H})^{+}$.

A solution of iodide $27 \mathrm{~g}$ ( $241 \mathrm{mg}, 0.44 \mathrm{mmol})$ in benzene $(17.0 \mathrm{~mL})$ was allowed to react with $\mathrm{Pd}_{2} \mathrm{dba}_{3} \cdot \mathrm{CHCl}_{3}(321 \mathrm{mg}, 0.59 \mathrm{mmol})$ as described for the preparation of 29 . Purification of the crude product by column chromatography (1:2 hexanes/benzene) provided 35 (364 mg, 94\%). Filtration through a plug of $\mathrm{SiO}_{2}\left(\mathrm{CH}_{2} \mathrm{Cl}_{2}\right)$ provided analytically pure 35 as a mixture of isomers, red flakes (345 mg, 90\%): $\mathrm{mp} 191{ }^{\circ} \mathrm{C}$; $[\square]_{\mathrm{D}}{ }^{26}$ $=+20.0^{\circ},[\square]_{577}{ }^{26}=+111.8^{\circ},[\square]_{546}{ }^{26}=+138.8^{\circ},[\square]_{435}{ }^{26}=+189.2^{\circ},\left(\mathrm{c}=0.2, \mathrm{CHCl}_{3}\right) ;{ }^{1} \mathrm{H}$ NMR (500 MHz, $\left.\mathrm{CDCl}_{3}\right) \square 7.58-7.61(\mathrm{~m}, 2 \mathrm{H})$, 7.22-7.26 (m, 2H), 6.90-6.95 (m, 2H), 6.81-6.84 (m, 2H), 5.04-5.06 (m, 2H), 4.64-4.67 (m, 2H), 4.55-4.57 (m, 2H), 4.45-4.50 (m, 10H), 3.90-3.92 (m, 2H), $3.68(\mathrm{~s}, 6 \mathrm{H}), 1.04(\mathrm{~s}, 18 \mathrm{H}) ;{ }^{13} \mathrm{C} \mathrm{NMR}\left(125 \mathrm{MHz}, \mathrm{CDCl}_{3}\right) \square$ 180.2, 179.8, 157.0, 132.2, 132.1, 128.2, 124.7, 120.0, 119.9, 110.43, 110.37, 98.7, 98.3, $83.6,83.5,78.2,77.5,74.5,73.5,73.0,72.9,71.5,71.0,69.8,55.6,55.5,35.70,35.66$, 27.3, 27.1 (not all carbon signals for the isomers are resolved); IR (film) 2961, $1583 \mathrm{~cm}^{-1}$; MS (FAB, MNBA) $m / z$ 1300, 649, 154; Anal. Calcd for $\mathrm{C}_{48} \mathrm{H}_{52} \mathrm{Fe}_{2} \mathrm{I}_{2} \mathrm{~N}_{2} \mathrm{O}_{4} \mathrm{Pd}_{2}: \mathrm{C}, 44.37 ; \mathrm{H}$, 4.03; N, 2.16. Found: C, 44.10; H, 3.87; N, 2.15. 


\section{$\left(R_{\mathrm{p}}\right)$-bis- $\square$-iodo-\{2-[(4S)-2-(4,5-dihydro-4-tert-butyl-2-oxazolyl)]-3-(4-methylsulfonyl-}

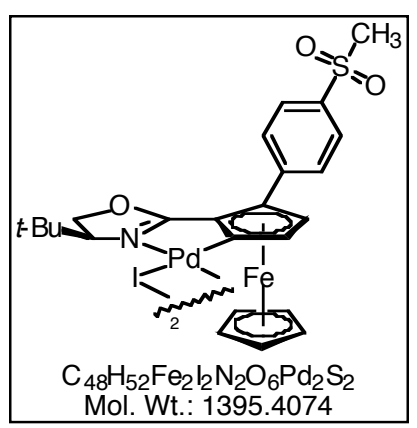

phenyl)-ferrocenyl dipalladium (36). A solution of iodide 27h (465 mg, $0.83 \mathrm{mmol})$ in $\mathrm{CH}_{2} \mathrm{Cl}_{2}(8.0 \mathrm{~mL})$ was cooled to 0 ${ }^{\circ} \mathrm{C}$ and $\mathrm{m}$-CPBA (450 $\left.\mathrm{mg}, 1.83 \mathrm{mmol}\right)$ was added in one portion. The solution was maintained at $0{ }^{\circ} \mathrm{C}$ for $30 \mathrm{~min}$ and quenched by addition of $10 \%$ aqueous $\mathrm{Na}_{2} \mathrm{~S}_{2} \mathrm{O}_{3}$ solution $(8 \mathrm{~mL})$. The biphasic mixture, while protected from light, was allowed

to warm to room temperature and stirred for $1 \mathrm{~h}$. The layers were separated and the aqueous phase was extracted with $\mathrm{CH}_{2} \mathrm{Cl}_{2}(10 \mathrm{~mL})$. The combined organic phases were washed with saturated aqueous $\mathrm{NaHCO}_{3}(10 \mathrm{~mL})$, dried $\left(\mathrm{MgSO}_{4}\right)$ and concentrated in vacuo to give a black tar. Purification by column chromatography (1:1 $\left.\mathrm{Et}_{2} \mathrm{O} / \mathrm{hexanes}\right)$ provided sulfone 28 (228 $\mathrm{mg}, 46 \%)$ as an unstable orange foam which was carried on directly: ${ }^{1} \mathrm{H} \mathrm{NMR}\left(400 \mathrm{MHz}, \mathrm{CDCl}_{3}\right.$ ) $\square 7.81$ (app dt, $J=8.6,1.9 \mathrm{~Hz}, 2 \mathrm{H}$ ), 7.73 (app dt, $J$ = 8.6, 1.9 Hz, 2H), $4.73(\mathrm{~d}, J=2.6 \mathrm{~Hz}, 1 \mathrm{H}), 4.64(\mathrm{~d}, J=2.6 \mathrm{~Hz}, 1 \mathrm{H}), 4.03(\mathrm{dd}, J=10.2$, $8.6 \mathrm{~Hz}, 1 \mathrm{H}), 4.23(\mathrm{~s}, 5 \mathrm{H}), 4.13(\operatorname{app~t}, J=8.7 \mathrm{~Hz}, 1 \mathrm{H}), 4.01(\mathrm{dd}, J=10.2,8.8 \mathrm{~Hz}, 1 \mathrm{H})$ 3.05 (s, 3H), 0.96 (s, 9H); IR (film) 2961, 1656, 1598, 1312, $1154 \mathrm{~cm}^{-1}$; LRMS (ES) $\mathrm{m} / \mathrm{z}$ $614(\mathrm{M}+\mathrm{Na})^{+}, 592(\mathrm{M}+\mathrm{H})^{+}$.

The above sulfone 28 was combined with $\mathrm{Pd}_{2} \mathrm{dba}_{3} \cdot \mathrm{CHCl}_{3}(269 \mathrm{mg}, 0.26 \mathrm{mmol})$ in benzene $(17 \mathrm{~mL})$ as described for the preparation of 29. Purification of the crude product by column chromatography $\left(\mathrm{CH}_{2} \mathrm{Cl}_{2}\right.$, then 5:95 $\left.\mathrm{Et}_{2} \mathrm{O} / \mathrm{CH}_{2} \mathrm{Cl}_{2}\right)$ provided $36(250 \mathrm{mg}$, $83 \%$ ). Recrystallization (benzene, room temperature) provided analytically pure $\mathbf{3 6}$ as a mixture of isomers, dark red prisms $(237 \mathrm{mg}, 79 \%)$ : $\mathrm{mp} 240{ }^{\circ} \mathrm{C}$; $[\square]_{\mathrm{D}}{ }^{26}=+100.5^{\circ},[\square]_{577}{ }^{26}$ $=+109.0^{\circ},[\square]_{546}{ }^{26}=+121.4^{\circ},[\square]_{435}{ }^{26}=-100.6^{\circ},[\square]_{405}{ }^{26}=-189.5^{\circ},\left(\mathrm{c}=0.30, \mathrm{CHCl}_{3}\right) ;{ }^{1} \mathrm{H}$ NMR (400 MHz, $\left.\mathrm{CDCl}_{3}\right) \square 7.80($ app t, $J=7.3 \mathrm{~Hz}, 4 \mathrm{H}), 7.68-7.70$ (m, 4H), 5.11-5.15 (m, 2H), 4.77-4.81 (m, 2H), 4.54-4.62 (m, 2H), $4.43(\mathrm{~s}, 5 \mathrm{H}), 4.39$ (s, 5H), 3.93-3.97 (m, 2H), $3.05(\mathrm{~m}, 6 \mathrm{H}), 1.02(\mathrm{~s}, 18 \mathrm{H}) ;{ }^{13} \mathrm{C} \mathrm{NMR}\left(125 \mathrm{MHz}, \mathrm{CDCl}_{3}\right) \square$ 179.4, 179.0, 144.1, 144.0, 138.0, 129.2, 129.1, 127.1, 127.0, 99.7, 99.4, 83.6, 80.1, 79.4, 74.8, 74.7, 73.5, $73.3,72.4,72.2,71.3,70.6,70.2,70.0,44.9,35.8,35.5,27.1,27.0$, (not all carbon signals for the two isomers are resolved); IR (film) 2961, $1586 \mathrm{~cm}^{-1}$; MS (FAB, MNBA) $\mathrm{m} / \mathrm{z}$ 
1395, 697, 154; Anal. Calcd for $\mathrm{C}_{48} \mathrm{H}_{52} \mathrm{Fe}_{2} \mathrm{I}_{2} \mathrm{~N}_{2} \mathrm{O}_{6} \mathrm{Pd}_{2} \mathrm{~S}_{2}$ : C, 41.32; H, 3.76; N, 2.01. Found: C, 41.18; H, 3.83; N, 2.03.

$N$-(2-Methoxyphenyl)benzimidoyl chloride (43c). $N$-(2-methoxyphenyl)-benzamide

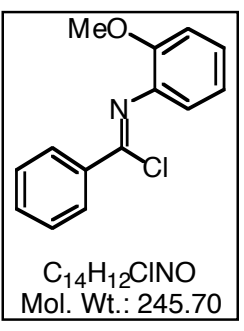
(42c) $(5.7 \mathrm{~g}, 25 \mathrm{mmol})$ and $\mathrm{PCl}_{5}(5.2 \mathrm{~g}, 25 \mathrm{mmol})$ were combined in a 25 $\mathrm{mL}$ round bottom flask connected in line to an empty trap and a trap containing saturated aqueous $\mathrm{NaHCO}_{3}$. The heterogeneous reaction was warmed to $80^{\circ} \mathrm{C}$ and became a homogenous solution. After 16 hours, the reaction was cooled to room temperature and $\mathrm{POCl}_{3}$ was removed in vасио. The residue was purified by Kugelrohr distillation $\left(10\right.$ torr, $\left.225^{\circ} \mathrm{C}\right)$ to give $4.97 \mathrm{~g}$ $(81 \%)$ of $42 \mathrm{c}$ as a viscous yellow oil: ${ }^{1} \mathrm{H}$ NMR $\left(500 \mathrm{MHz}, \mathrm{CDCl}_{3}\right) \square 8.19-8.23(\mathrm{~m}, 2 \mathrm{H})$, $7.55(\mathrm{tt}, J=7.2,1.3 \mathrm{~Hz}, 1 \mathrm{H}), 7.46-7.52(\mathrm{~m}, 2 \mathrm{H}), 7.16-7.23(\mathrm{~m}, 1 \mathrm{H}), 6.97-7.03(\mathrm{~m}, 2 \mathrm{H})$, 6.91-6.96 (m, 1H), $3.86(\mathrm{~s}, 3 \mathrm{H}) ;{ }^{13} \mathrm{C}$ NMR (125 MHz, $\left.\mathrm{CDCl}_{3}\right) \square$ 149.9, 145.2, 137.5, $135.7,132.3,129.8,128.7,126.2,121.1,120.9,112.0,56.1$; IR (neat) 3005, 2960, 2836, $1657,1591,1494,1257,1169,1114,1026 \mathrm{~cm}^{-1}$.

$N$-(2,4-Dimethoxylphenyl)benzimidoyl chloride (43d). Benzoic acid (4.9 g, 40 mmol),

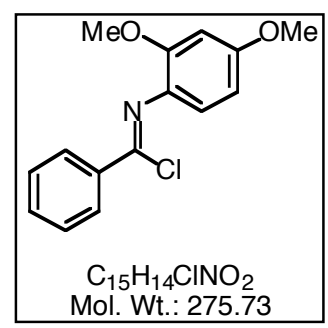

$\mathrm{PPh}_{3}(31.5 \mathrm{~g}, 120 \mathrm{mmol}), \mathrm{Et}_{3} \mathrm{~N}(6.7 \mathrm{~mL}, 48 \mathrm{mmol})$ and $\mathrm{CCl}_{4}(20 \mathrm{~mL})$ were cooled to $0{ }^{\circ} \mathrm{C}$ for 10 minutes. 2,4-Dimethoxyaniline $(7.4 \mathrm{~g}, 48$ mmol) in $\mathrm{CCl}_{4}(15 \mathrm{~mL})$ was added to the reaction. The mixture was warmed to reflux and maintained for 18 hours. After cooling to room temperature, the volatiles were removed in vacuo, and the residue slurried in hexanes $(250 \mathrm{~mL})$. The slurry was stirred vigorously for 3 hours and filtered. Concentration of the mother liquor in vacuo afforded $4.22 \mathrm{~g}$ (38\%) of imidoyl chloride 43d as a yellow oil: ${ }^{1} \mathrm{H}$ NMR (500 $\left.\mathrm{MHz}, \mathrm{CDCl}_{3}\right) \square 8.17-8.21(\mathrm{~m}, 2 \mathrm{H}), 7.50-7.56$ (m, 1H), 7.44-7.50 (m, 2H), $6.94(\mathrm{~d}, J=8.5 \mathrm{~Hz}, 1 \mathrm{H}), 6.57(\mathrm{~d}, J=2.5 \mathrm{~Hz}, 1 \mathrm{H}), 6.54(\mathrm{dd}, J=$ 8.6, $2.5 \mathrm{~Hz}, 1 \mathrm{H}), 3.84(\mathrm{~s}, 3 \mathrm{H}), 3.82(\mathrm{~s}, 3 \mathrm{H}) ;{ }^{13} \mathrm{C} \mathrm{NMR}\left(125 \mathrm{MHz}, \mathrm{CDCl}_{3}\right) \square$ 158.4, 151.2, $135.5,131.9,129.5,128.7,128.4,127.0,121.5,104.0,99.6,55.8,55.5$; IR (neat) 3064 , $3002,2939,2837,1659,1605,1582,1526,1416,1282,1207,1173,1126,1033 \mathrm{~cm}^{-1}$. 
trans-2-Hexenyl- $N$-(2-methoxyphenyl)benzimidate (5c). Sodium hydride (41 mg, 1.0

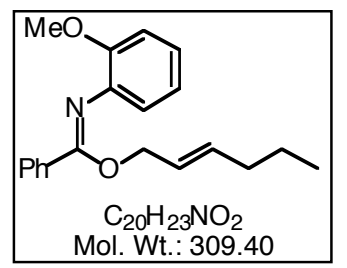
$\mathrm{mmol})$ and THF (1 mL) were cooled to $0{ }^{\circ} \mathrm{C}$ and trans-2-hexen-1-ol (0.12 $\mathrm{mL}, 1.0 \mathrm{mmol})$ was added dropwise. The reaction was warmed to room temperature over 4 hours before adding $\mathrm{N}$-(2methoxybenzyl)benzimidoyl chloride $(43 \mathrm{c}, 250 \mathrm{mg}, 1.0 \mathrm{mmol})$ in THF (1 mL). The reaction was maintained for 12 hours before adding EtOAc $(10 \mathrm{~mL})$ and $\mathrm{H}_{2} \mathrm{O}(5 \mathrm{~mL})$. The phases were separated and the aqueous phase was extracted with EtOAc $(2 \times 5 \mathrm{~mL})$. The combined organic phase was dried $\left(\mathrm{MgSO}_{4}\right)$ and concentrated in vасио. Purification by chromatography (95:5 hexanes/EtOAc) yielded $220 \mathrm{mg}$ (70\%) of imidate 5c as a pale yellow oil: ${ }^{1} \mathrm{H}$ NMR $\left(500 \mathrm{MHz}, \mathrm{CDCl}_{3}\right) \square 7.31(\mathrm{~d}, J=10.4 \mathrm{~Hz}, 2 \mathrm{H})$, 7.25 (tt, $J=7.4,1.3 \mathrm{~Hz}, 1 \mathrm{H}), 7.19$ (app t, $J=7.4 \mathrm{~Hz}, 2 \mathrm{H}), 6.90-6.95 \mathrm{~Hz}, 2 \mathrm{H}), 3.64$ (s, 3H), 2.08 (app q, $J=7.2 \mathrm{~Hz}, 2 \mathrm{H}$ ), 1.45 (sextet, $J=7.4 \mathrm{~Hz}, 2 \mathrm{H}$ ), 0.93 (t, $J=7.4 \mathrm{~Hz}$, $3 \mathrm{H}) ;{ }^{13} \mathrm{C}$ NMR $\left(125 \mathrm{MHz}, \mathrm{CDCl}_{3}\right) \square 160.0,150.7,138.5,135.8,132.8,130.1,128.9$, $128.1,125.3,123.8,122.9,121.4,112.1$, 67.9, 55.9, 34.9, 22.6, 14.1; IR (neat) 3062, 2958, 2874, 1660, 1593, 1495, 1447, 1266, 1109, $1028 \mathrm{~cm}^{-1}$; LRMS (ES) $\mathrm{m} / \mathrm{z} 332.26$ (M $+\mathrm{Na})^{+}$. Anal. Calcd for $\mathrm{C}_{20} \mathrm{H}_{22} \mathrm{NO}_{2}: \mathrm{C}, 77.64 ; \mathrm{H}, 7.49 ; \mathrm{N}, 4.53$. Found: C, 77.82; H, $7.48 ; \mathrm{N}, 4.68$.

trans-2-Hexenyl- $N$-(2,4-dimethoxyphenyl)benzimidate (5d). trans-2-Hexen-1-ol (0.11

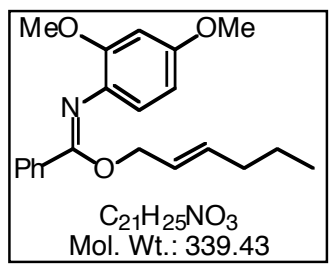
$\mathrm{mL}, 0.91 \mathrm{mmol})$ in THF $(1.7 \mathrm{~mL})$ was cooled to $0{ }^{\circ} \mathrm{C}$ and $n$-BuLi (2.5 M, $0.35 \mathrm{~mL}, 0.88 \mathrm{mmol})$ was added dropwise. The reaction was stirred at $0{ }^{\circ} \mathrm{C}$ for 15 minutes and then at room temperature for 30 minutes before adding $N$-(2,4-dimethoxyphenyl)benzimidoyl chloride (43d, $200 \mathrm{mg}, 0.73 \mathrm{mmol})$ in THF $(2.0 \mathrm{~mL})$. The reaction was maintained for 12 hours before adding EtOAc $(10 \mathrm{~mL})$ and $\mathrm{H}_{2} \mathrm{O}(5 \mathrm{~mL})$. The phases were separated and the aqueous phase was extracted with EtOAc $(2 \times 10 \mathrm{~mL})$. The combined organic phases were dried $\left(\mathrm{Na}_{2} \mathrm{SO}_{4}\right)$ and concentrated in vacuo. Purification by column chromatography (19:1 hexanes/EtOAc) yielded $67 \mathrm{mg}(27 \%)$ of imidate $\mathbf{5 d}$ as a 
yellow oil: $\mathrm{R}_{\mathrm{f}}=0.29$ (4:1 hexanes/EtOAc); ${ }^{1} \mathrm{H} \mathrm{NMR}\left(500 \mathrm{MHz}, \mathrm{CDCl}_{3}\right) \square 7.24-7.33(\mathrm{~m}$, $3 \mathrm{H}), 7.20(\mathrm{t}, J=7.4 \mathrm{~Hz}, 2 \mathrm{H}), 6.54(\operatorname{app~d}, J=8.3 \mathrm{~Hz}, 1 \mathrm{H}), 6.38(\mathrm{~d}, J=2.4 \mathrm{~Hz}, 1 \mathrm{H}), 6.31$ $(\mathrm{dd}, J=8.5,2.5 \mathrm{~Hz}, 1 \mathrm{H}), 5.75-5.90(\mathrm{~m}, 2 \mathrm{H}), 4.84(\mathrm{~d}, J=4.6 \mathrm{~Hz}, 2 \mathrm{H}), 3.74(\mathrm{~s}, 3 \mathrm{H}), 3.60$ (s, 3H), 2.08 (q, $J=7.0 \mathrm{~Hz}, 2 \mathrm{H}), 1.44$ (sextet, $J=7.4 \mathrm{~Hz}, 2 \mathrm{H}), 0.93$ (t, $J=7.4 \mathrm{~Hz}, 3 \mathrm{H}$ ); ${ }^{13} \mathrm{C}$ NMR (125 MHz, $\left.\mathrm{CDCl}_{3}\right) \square 159.9,156.3,151.0,135.3,132.5,131.5,129.5,128.4$, $127.7,125.0,122.5,104.2,99.7,67.3,55.43,55.40,34.5,22.2,13.7$; IR (neat) 2961, 2934, 2837, 1660, 1606, 1505, 1463, 1270, 1208, 1158, 1112, $1042 \mathrm{~cm}^{-1}$; LRMS (ES) $\mathrm{m} / \mathrm{z}$ $362.28(\mathrm{M}+\mathrm{Na})^{+}$. Anal. Calcd for $\mathrm{C}_{20} \mathrm{H}_{22} \mathrm{NO}_{2}: \mathrm{C}, 74.31 ; \mathrm{H}, 7.42 ; \mathrm{N}, 4.13$. Found: $\mathrm{C}$, 74.41; H, 7.60; N, 4.00.

Methyl- $N$-(benzyloxycarbonyl)benzimidate (45). A biphasic mixture of saturated

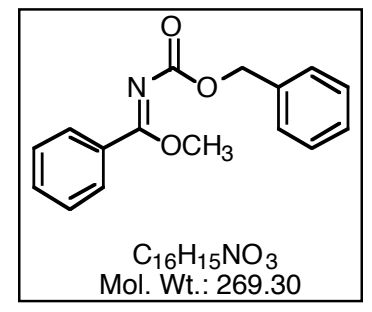
aqueous $\mathrm{NaHCO}_{3}(20 \mathrm{~mL})$ and pentane $(20 \mathrm{~mL})$ was cooled to 0 ${ }^{\circ} \mathrm{C}$ in a separatory funnel. $O$-Methylbenzimidate hydrochloride ${ }^{3}$ (44) (400 mg, $2.34 \mathrm{mmol})$ was added and shaken vigorously for 2 minutes. The layers were separated and the organic phase was dried $\left(\mathrm{Na}_{2} \mathrm{SO}_{4}\right)$, and concentrated in vacuo. The residue was dissolved in $\mathrm{CH}_{2} \mathrm{Cl}_{2}(2 \mathrm{~mL})$ and $\mathrm{K}_{2} \mathrm{CO}_{3}(311 \mathrm{mg}, 2.26 \mathrm{mmol})$ was added. Benzyl chloroformate $(0.39 \mathrm{~mL}, 2.71 \mathrm{mmol})$ was added dropwise (caution; gas evolution) and the reaction was maintained at $23{ }^{\circ} \mathrm{C}$ for 16 hours before concentrating in vacuo. Purification by flash column chromatography (19:1 hexanes/EtOAc) gave $280 \mathrm{mg}(46 \%)$ of $\mathbf{4 5}$ as a pale yellow oil: $\mathrm{R}_{\mathrm{f}}=0.48(4: 1$ hexanes/EtOAc); ${ }^{1} \mathrm{H}$ NMR $\left(500 \mathrm{MHz}, \mathrm{CDCl}_{3}\right) \square 7.43(\mathrm{td}, J=7.2,1.3 \mathrm{~Hz}, 2 \mathrm{H}), 7.32$ (td, $J$ $=7.5,1.2 \mathrm{~Hz}, 1 \mathrm{H}), 7.12-7.27(7 \mathrm{H}, \mathrm{m}), 5.04(\mathrm{~s}, 2 \mathrm{H}), 3.82(\mathrm{~s}, 3 \mathrm{H}) ;{ }^{13} \mathrm{C} \mathrm{NMR}(125 \mathrm{MHz}$, $\left.\mathrm{CDCl}_{3}\right) \square 163.5,160.7,135.4,131.6,130.7,128.6,128.4$ (2 overlapping signals), 128.2, 127.8, 68.2, 55.1; IR (film) 3064, 2949, 1715, 1667, 1450, 1263, 1178, 1121, $1024 \mathrm{~cm}^{-1}$; LRMS (ES) $m / z \quad 292.10(\mathrm{M}+\mathrm{Na})^{+}$. Anal. Calcd for $\mathrm{C}_{16} \mathrm{H}_{15} \mathrm{NO}_{3}: \mathrm{C}, 71.36 ; \mathrm{H}, 5.61 ; \mathrm{N}$, 5.20. Found: C, 71.43; H, 5.77; N, 5.20.

\footnotetext{
${ }^{3}$ Casy, G.; Patterson, J. W.; Taylor, R. J. K. Org. Synth. 1988, 67, 193-197.
} 
trans-2-Hexenyl- $N$-(benzyloxycarbonyl)benzimidate (46). A $100 \mathrm{~mL}$ round bottom

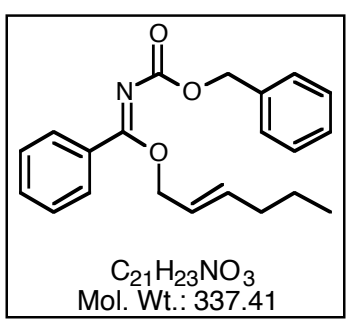
flask equipped with a $7 \mathrm{~cm}$ vigreux column and a short path distillation head was charged with imidate 45 (120 mg, 0.45 $\mathrm{mmol})$ and benzene $(60 \mathrm{~mL})$. Trans-2-hexen-1-ol (80 $\square \mathrm{L}, 0.68$ mmol) and potassium tert-butoxide $(1.5 \mathrm{mg}, 0.014 \mathrm{mmol})$ were added. The reaction was warmed to $87^{\circ} \mathrm{C}$ and was distilled to a volume of $3 \mathrm{~mL}$. The reaction was monitored by TLC and additional portions of benzene $(60 \mathrm{~mL})$ were added and distilled to a minimal volume until no starting material remained. The residue was concentrated in vacuo and purified by column chromatography (19:1 hexanes/EtOAc) to afford $64 \mathrm{mg}$ (42\%) of $\mathbf{4 6}$ as a pale yellow oil. An analytical sample of $\mathbf{4 6}$ was prepared by column chromatography $(2: 1$ benzene/hexanes): $\mathrm{R}_{\mathrm{f}}=0.58$ (4:1 hexanes/EtOAc); ${ }^{1} \mathrm{H}$ NMR $\left(500 \mathrm{MHz}, \mathrm{CDCl}_{3}\right) \square 7.53$ (dd, $J=7.3,0.8 \mathrm{~Hz}, 2 \mathrm{H}), 7.43(\mathrm{qt}, J=7.5,0.7 \mathrm{~Hz}, 1 \mathrm{H}), 7.20-7.32(\mathrm{~m}, 7 \mathrm{H}), 5.81-5.88(\mathrm{~m}$, 1H), 5.66-5.74 (m, 1H), $5.13(\mathrm{~s}, 2 \mathrm{H}), 4.73(\mathrm{~d}, J=6.5 \mathrm{~Hz}, 2 \mathrm{H}), 2.06(\mathrm{q}, J=7.1 \mathrm{~Hz}, 2 \mathrm{H})$, 1.43 (sextet, $J=7.4 \mathrm{~Hz}, 2 \mathrm{H}), 0.91(\mathrm{t}, J=7.4 \mathrm{~Hz}, 3 \mathrm{H}) ;{ }^{13} \mathrm{C} \mathrm{NMR}\left(100 \mathrm{MHz}, \mathrm{CDCl}_{3}\right) \square$ $162.8,160.5,136.9,135.4,131.4,131.0,128.5,128.35,128.34,128.1,127.8,123.4,68.8$, 68.0, 34.3, 22.0, 13.5; IR (film) 3063, 2958, 2872, 1716, 1660, 1450, 1253, $1119 \mathrm{~cm}^{-1}$; LRMS (ES) $m / z 360.09(\mathrm{M}+\mathrm{Na})^{+}$. Anal. Calcd for $\mathrm{C}_{16} \mathrm{H}_{15} \mathrm{NO}_{3}: \mathrm{C}, 74.75 ; \mathrm{H}, 6.87 ; \mathrm{N}$, 4.15. Found: C, 74.88; H, 6.95; N, 4.10.

Trans-2-Hexenyl- $N$-(2-hexenyloxycarbonyl)benzimidate (47) formed during the

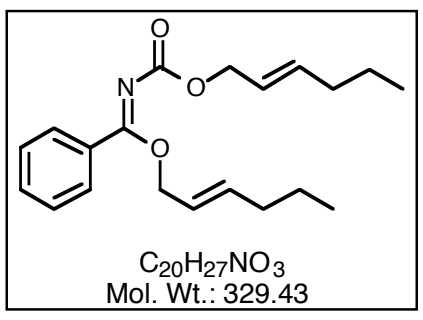
conversion of 45 to 46. $\mathrm{R}_{\mathrm{f}}=0.68\left(4: 1\right.$ hexanes/EtOAc); ${ }^{1} \mathrm{H}$ NMR (500 MHz, $\left.\mathrm{CDCl}_{3}\right) \square 7.61-7.64(\mathrm{~m}, 2 \mathrm{H}), 7.47(\mathrm{tt}, J=$ 6.2, $1.3 \mathrm{~Hz}, 1 \mathrm{H}), 7.36-7.41(\mathrm{~m}, 2 \mathrm{H}), 5.80-5.89(\mathrm{~m}, 1 \mathrm{H})$, 5.67-5.75 (m, 2H), 5.50 (dddd, $J=14.3,6.6,6.6,1.5 \mathrm{~Hz}, 1 \mathrm{H})$, $4.73(\mathrm{dd}, J=6.4,0.9 \mathrm{~Hz}, 2 \mathrm{H}), 4.54(\mathrm{dd}, J=6.6,0.9 \mathrm{~Hz}, 2 \mathrm{H})$, $2.06(\mathrm{q}, J=6.8 \mathrm{~Hz}, 2 \mathrm{H}), 1.98(\mathrm{q}, J=6.8 \mathrm{~Hz}, 2 \mathrm{H}), 1.42$ (sextet, $J=7.4 \mathrm{~Hz}, 2 \mathrm{H}), 1.37$ (sextet, $J=7.3 \mathrm{~Hz}, 2 \mathrm{H}), 0.92(\mathrm{t}, J=7.3 \mathrm{~Hz}, 3 \mathrm{H}), 0.89(\mathrm{t}, J=7.4 \mathrm{~Hz}, 3 \mathrm{H}) ;{ }^{13} \mathrm{C} \mathrm{NMR}(125$ MHz, acetone-d $\left.\mathrm{d}^{6}\right) \square 161.8,160.3,136.7,136.3,132.0,131.8,129.0,128.2,124.6,124.4$, 
68.5, 66.7, 34.5, 34.4, 22.32, 22.25, 13.4; IR (film) 2959, 2931, 2873, 1720, 1665 1252, $1119 \mathrm{~cm}^{-1}$; HRMS (EI) $\mathrm{m} / z$ calcd for $\mathrm{C}_{20} \mathrm{H}_{27} \mathrm{NO}_{3}(\mathrm{M})^{+} 329.1991$, found 329.1983.

Methyl- $N$-(tert-butylsulfonyl)benzimidate (49). Tert-butylsulfonamide (48) $(800 \mathrm{mg}$,

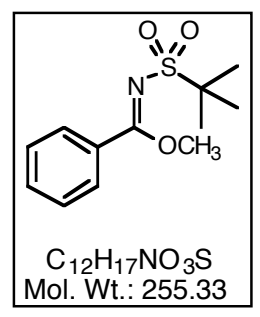

hexanes/EtOAc) gave $713 \mathrm{mg}(48 \%)$ of 49 as a white solid: $\mathrm{R}_{\mathrm{f}}=0.26(4: 1$ hexanes/EtOAc); mp: $67-69{ }^{\circ} \mathrm{C} ;{ }^{1} \mathrm{H}$ NMR $\left(500 \mathrm{MHz}, \mathrm{CDCl}_{3}\right) \square 7.90$ (tt, $J=7.2,1.3 \mathrm{~Hz}$, 2H), 7.53 (tt, $J=7.5,1.2 \mathrm{~Hz}, 1 \mathrm{H}), 7.44$ (tt, $J=7.5,1.6 \mathrm{~Hz}, 2 \mathrm{H}), 3.98$ (s, 3H), 1.49 (s, 9H); ${ }^{13} \mathrm{C}$ NMR $\left(125 \mathrm{MHz}, \mathrm{CDCl}_{3}\right) \square 170.9,132.4,131.2,129.3,129.0,59.1,55.7,24.2$; IR (film) 3068, 2986, 1614, 1437, 1298, $1121 \mathrm{~cm}^{-1}$; LRMS (ES) $m / z 278.09(\mathrm{M}+\mathrm{Na})^{+}$. Anal. Calcd for $\mathrm{C}_{16} \mathrm{H}_{15} \mathrm{NO}_{3}$ : C, 56.45; H, 6.71; N, 5.49. Found: C, 56.66; H, 6.73; N, 5.39 .

trans-2-Hexenyl- $N$-(tert-butylsulfonyl)benzimidate (50). A $100 \mathrm{~mL}$ round bottom flask Mol. Wt.: 323.45 equipped with a $7 \mathrm{~cm}$ vigreux column and a short path distillation head was charged with imidate $49(263 \mathrm{mg}, 1.03 \mathrm{mmol})$ and benzene (60 mL). Trans-2-hexen-1-ol (0.36 mL, $3.09 \mathrm{mmol})$ and potassium tert-butoxide $(23 \mathrm{mg}, 0.21 \mathrm{mmol})$ were added. The reaction was warmed to $100^{\circ} \mathrm{C}$ and distilled to a volume of $3 \mathrm{~mL}$. hexanes/EtOAc) afforded $225 \mathrm{mg}(68 \%)$ of $\mathbf{5 0}$ as a white solid: $\mathrm{R}_{\mathrm{f}}=0.42$ (4:1 hexanes/EtOAc); mp: $37-39{ }^{\circ} \mathrm{C} ;{ }^{1} \mathrm{H}$ NMR $\left(500 \mathrm{MHz}, \mathrm{CDCl}_{3}\right) \square 7.90(\mathrm{td}, J=7.2,1.3 \mathrm{~Hz}$, 2H), $7.52(\mathrm{tt}, J=7.5,1.0 \mathrm{~Hz}, 1 \mathrm{H}), 7.44(\mathrm{tt}, J=7.9,1.7 \mathrm{~Hz}, 2 \mathrm{H}), 5.85-5.92(\mathrm{~m}, 1 \mathrm{H})$, 5.66-5.73 (m, 1H), 4.78 (dd, $J=6.5,0.9 \mathrm{~Hz}, 2 \mathrm{H}), 2.07$ (q, $J=7.0 \mathrm{~Hz}, 2 \mathrm{H}), 1.49$ (s, 9H), 1.42 (sextet, $J=7.4 \mathrm{~Hz}, 2 \mathrm{H}), 0.92(\mathrm{t}, J=7.4 \mathrm{~Hz}, 3 \mathrm{H}) ;{ }^{13} \mathrm{C} \mathrm{NMR}\left(125 \mathrm{MHz}, \mathrm{CDCl}_{3}\right) \square$ 
$170.4,137.7,132.3,131.5,129.3,128.0,122.8,69.5,59.0,34.3,24.2,21.9,13.6$; IR (film) 2958, 1598, 1579, 1299, 1273, $1122 \mathrm{~cm}^{-1}$; LRMS (ES) $m / z 346.09(\mathrm{M}+\mathrm{Na})^{+}$. Anal. Calcd for $\mathrm{C}_{16} \mathrm{H}_{15} \mathrm{NO}_{3}$ : C, 63.13; H, 7.79; N, 4.33. Found: C, 63.40; H, 7.95; N, 4.25 .

(S)- $N$-(2-Methoxyphenyl)- $N$-(1-ethenylbutyl)benzamide (6c). Palladium iodide

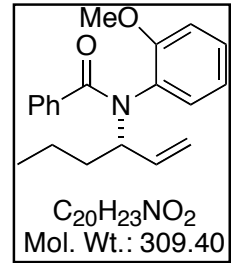
catalyst 9a (14 mg, $0.011 \mathrm{mmol})$ and $\mathrm{AgOCOCF}_{3}(10 \mathrm{mg}, 0.044 \mathrm{mmol})$ were dissolved in $\mathrm{CH}_{2} \mathrm{Cl}_{2}(0.5 \mathrm{~mL})$ under argon and protected from light. After 3 hours, the solution containing the activated catalyst was filtered through Celite and rinsed with $\mathrm{CH}_{2} \mathrm{Cl}_{2}(0.5 \mathrm{~mL})$ while under argon and added directly to a solution of imidate $5 \mathbf{c}(67 \mathrm{mg}, 0.22 \mathrm{mmol})$ and $\mathrm{CH}_{2} \mathrm{Cl}_{2}(0.5 \mathrm{~mL})$. Purification by column chromatography (19:1 hexanes/EtOAc) afforded $44 \mathrm{mg}$ (66\%) of 6c as a white solid. Separation of enantiomers by chiral HPLC (Chiracel OD-H, 98:2 nhexanes/IPA, $1 \mathrm{~mL} / \mathrm{min}$ ) showed that $\mathbf{6 c}$ had been formed in $77 \%$ ee: mp: $52-54{ }^{\circ} \mathrm{C}$; $[\square]_{D}^{25}=+16.1,[\square]_{577}^{25}=+17.9,[\square]_{546}^{25}=+20.2,[\square]_{435}^{25}=+38.9,[\square]_{405}^{25}=+49.0(\mathrm{c} 0.8$, $\left.\mathrm{CHCl}_{3}\right) ;{ }^{1} \mathrm{H} \mathrm{NMR}\left(500 \mathrm{MHz}, \mathrm{CDCl}_{3}, 1: 1\right.$ mixture of rotamers) $\square 7.25-7.30(\mathrm{~m}, 4 \mathrm{H})$, 7.05-7.20 (m, 10H), 6.78-6.85 (m, 2H), 6.63 (app t, $J=6.8 \mathrm{~Hz}, 2 \mathrm{H}), 5.95$ (ddd, $J=17.9$, $10.1,7.9 \mathrm{~Hz}, 1 \mathrm{H}), 5.76(\mathrm{ddd}, J=17.5,10.1,7.8 \mathrm{~Hz}, 1 \mathrm{H}), 5.32(\mathrm{~d}, J=17.2 \mathrm{~Hz}, 1 \mathrm{H}), 5.21$ $(\mathrm{d}, J=10.2 \mathrm{~Hz}, 1 \mathrm{H}), 5.04-5.16(\mathrm{~m}, 3 \mathrm{H}), 5.00(\mathrm{~d}, J=10.3 \mathrm{~Hz}, 1 \mathrm{H}), 3.59(\mathrm{~s}, 3 \mathrm{H}), 3.55(\mathrm{~s}$, 3H), 1.85-1.95 (m, 1H), 1.66-1.82 (m, 2H), 1.46-1.56 (m, 2H), 1.33-1.45 (m, 3H), 1.00 $(\mathrm{t}, J=7.3 \mathrm{~Hz}, 3 \mathrm{H}), 0.85-0.92(\mathrm{~m}, 3 \mathrm{H}) ;{ }^{13} \mathrm{C} \mathrm{NMR}\left(125 \mathrm{MHz}, \mathrm{CDCl}_{3}, 1: 1\right.$ mixture of rotamers) $\square 171.7,171.4,155.5,155.4,138.1,137.4$ (broad), 136.6, 130.8, 130.42, $130.37,130.2$, 128.9, 128.8 (broad), 128.7, 127.8, 127.7, 127.0, 126.9, 120.1, 120.0, $117.1,116.7,111.5,111.4,60.0,59.9,55.0,54.9,35.1,33.3,19.8,19.6,14.01,13.96$; IR (neat) 3067, 2960, 2873, 1646, 1596, 1498, 1464, 1377, 1327, 1278, 1161, $1027 \mathrm{~cm}^{-1}$; HRMS (EI) $m / z$ calcd for $\mathrm{C}_{20} \mathrm{H}_{23} \mathrm{NO}_{2}(\mathrm{M})^{+} 309.1729$, found 309.1722 . 
(S)- $N$-(2,4-Dimethoxyphenyl)- $N$-(1-ethenylbutyl)benzamide (6d). Following the

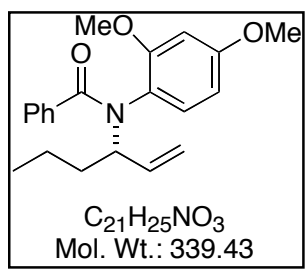
general procedure for palladium iodide precatalyst activation, 9a (3.1 $\mathrm{mg}, 0.0025 \mathrm{mmol})$ was activated with $\mathrm{AgOCOCF}_{3}(3 \mathrm{mg}, 0.014$ mmol) to provide a solution containing the active catalyst. Imidate $\mathbf{5 d}$ $(17 \mathrm{mg}, 0.05 \mathrm{mmol})$ was treated the solution containing the active catalyst to afford, after chromatography (19:1 hexanes/EtOAc), $15 \mathrm{mg}(88 \%)$ of a 3:1 mixture of $\mathbf{6 d}$ and 5d. Separation of enantiomers by chiral HPLC (Chiracel OD-H, 98:2 n-hexanes/IPA, $1 \mathrm{~mL} / \mathrm{min})$ showed that $\mathbf{6 d}$ had been formed in $83 \%$ ee: $\mathrm{R}_{\mathrm{f}}=0.26(4: 1$ hexanes/EtOAc); ${ }^{1} \mathrm{H}$ NMR (500 MHz, $\mathrm{CDCl}_{3}, 1: 1$ mixture of rotamers) $\square 7.24-7.29$ (m, 4H), 7.06-7.17 (m, 6H), 7.04 (d, J=8.6 Hz, 1H), 6.96 (d, J=8.6 Hz, 1H), 6.29-6.35 (m, 2H), 6.16-6.21 (m, 2H), 5.89 (ddd, $J=17.5,10.1,7.9 \mathrm{~Hz}, 1 \mathrm{H}), 5.72$ (ddd, $J=17.6,10.2$, $7.9 \mathrm{~Hz}, 1 \mathrm{H}), 5.30(\mathrm{~d}, J=17.1 \mathrm{~Hz}, 1 \mathrm{H}), 5.20(\mathrm{~d}, J=10.3 \mathrm{~Hz}, 1 \mathrm{H}), 5.04-5.16(\mathrm{~m}, 3 \mathrm{H})$, $5.00(\mathrm{~d}, J=10.4 \mathrm{~Hz}, 1 \mathrm{H}), 3.72(\mathrm{~s}, 3 \mathrm{H}), 3.71(\mathrm{~s}, 3 \mathrm{H}), 3.57$ (s, 3H), 3.55 (s, 3H), 1.80-1.89 (m, 1H), 1.64-1.77 (m, 2H), 1.45-1.54 (m, 2H), 1.32-1.42 (m, 3H), 0.99 (t, $J=7.3 \mathrm{~Hz}$, $3 \mathrm{H}), 0.89(\mathrm{t}, J=7.0 \mathrm{~Hz}, 3 \mathrm{H}) ;{ }^{13} \mathrm{C}$ NMR $\left(125 \mathrm{MHz}, \mathrm{CDCl}_{3}, 1: 1\right.$ mixture of rotamers) $\square$ 171.6, 160.0, 159.9, 156.4, 138.2, 137.6, 136.7, 131.5, 131.4, 130.8, 128.8, 128.7, 127.7, 127.6, 127.1, 127.04, 126.97, 117.7, 116.6, 103.8, 103.7, 98.9, 98.8, 98.7, 59.7, 59.6, $55.30,55.29,55.0,54.9,35.0,33.3,19.8,19.6,14.1,14.0$; IR (neat) 3080, 2961, 2934, 2872, 1644, 1509, 1309, 1212, 1162, $1034 \mathrm{~cm}^{-1}$; HRMS (CI) $\mathrm{m} / z$ calcd for $\mathrm{C}_{21} \mathrm{H}_{25} \mathrm{NO}$ $(\mathrm{M})^{+} 339.1834$, found 339.1834 .

$N$-(benzyloxycarbonyl)- $N$-(1-ethenylbutyl)benzamide (53). Following the general

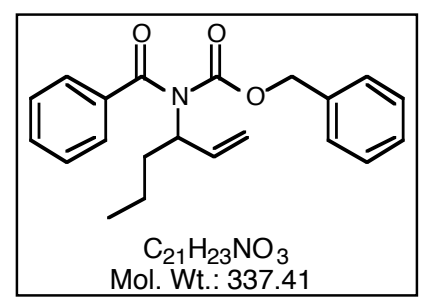

procedure for palladium iodide precatalyst activation, 9a $(9.2$ $\mathrm{mg}, 0.0075 \mathrm{mmol})$ was activated with $\mathrm{AgOCOCF}_{3}(6.6 \mathrm{mg}$, $0.03 \mathrm{mmol})$ to provide a solution containing the activated catalyst. Imidate $46(50 \mathrm{mg}, 0.15 \mathrm{mmol})$ was treated with the solution containing the activated catalyst to afford, after purification by flash column chromatography (19:1 hexanes/EtOAc), $34 \mathrm{mg}(68 \%)$ of $\mathbf{5 3}$ : $\mathrm{R}_{\mathrm{f}}=0.62\left(4: 1\right.$ hexanes/EtOAc); ${ }^{1} \mathrm{H} \mathrm{NMR}\left(500 \mathrm{MHz}, \mathrm{CDCl}_{3}\right) \square 7.58(\mathrm{td}, J=7.1,1.1 \mathrm{~Hz}$, 
2H), $7.48(\mathrm{tt}, J=7.4,1.3 \mathrm{~Hz}, 1 \mathrm{H}), 7.37(\mathrm{tt}, J=7,5,1.6 \mathrm{~Hz}, 2 \mathrm{H}), 7.28(\mathrm{tt}, J=7.3,1.4 \mathrm{~Hz}$, 1H), 7.23 (tt, $J=7.0,1.3 \mathrm{~Hz}, 2 \mathrm{H}), 6.85$ (tt, $J=7.2,1.5 \mathrm{~Hz}, 2 \mathrm{H}), 6.19$ (ddd, $J=17.4,10.3$, $7.3 \mathrm{~Hz}, 1 \mathrm{H}), 5.28$ (td, $J=17.3,1.3 \mathrm{~Hz}, 1 \mathrm{H}), 5.19$ (td, $J=10.3,1.2 \mathrm{~Hz}, 1 \mathrm{H}), 4.94-4.99$ (m, 1H), $4.94(\mathrm{~s}, 2 \mathrm{H}), 1.98-2.08(\mathrm{~m}, 1 \mathrm{H}), 1.81-1.89(\mathrm{~m}, 1 \mathrm{H}), 1.37-1.44(\mathrm{~m}, 2 \mathrm{H}), 0.96(\mathrm{t}$, $J=7.3 \mathrm{~Hz}, 3 \mathrm{H}) ;{ }^{13} \mathrm{C} \mathrm{NMR}\left(125 \mathrm{MHz}, \mathrm{CDCl}_{3}\right) \square 173.0,155.2,137.6,137.5,134.6,131.8$, 128.6, 128.54 (2 overlapping signals), 128.47, 127.9, 117.6, 68.7, 60.3, 34.7, 20.0, 14.0; IR (film) 2958, 1736, 1677, 1383, 1326, $1221 \mathrm{~cm}^{-1}$; LRMS (ES) $m / z, 360.09(\mathrm{M}+\mathrm{Na})^{+}$. Anal. Calcd for $\mathrm{C}_{16} \mathrm{H}_{15} \mathrm{NO}_{3}$ : C, 74.75; H, 6.87; N, 4.15. Found: C, 74.93; H, 6.99; N, 4.11 .

$N$-(4-Methoxyphenyl)- $N$-(1-ethenylbutyl)trifluoroacetamide (55a). Following the

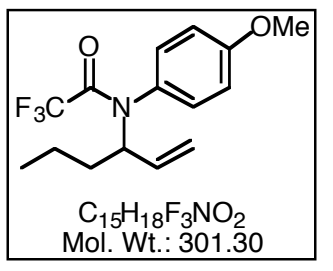
general procedure for palladium iodide precatalyst activation, 9a (15 mg, $0.012 \mathrm{mmol})$ was treated with $\mathrm{AgOCOCF}_{3}(11 \mathrm{mg}, 0.048$ mmol) to provide a solution containing the activated catalyst $(0.03$ M) in $\mathrm{CH}_{2} \mathrm{Cl}_{2}$. Imidate $52 \mathrm{a}(25 \mathrm{mg}, 0.08 \mathrm{mmol})$ was diluted in $\mathrm{CH}_{2} \mathrm{Cl}_{2}(0.2 \mathrm{~mL})$ and the solution containing the activated catalyst $(0.03 \mathrm{M}, 0.13 \mathrm{~mL}$, $0.006 \mathrm{mmol})$ was added followed by proton sponge ${ }^{\circledR}(3 \mathrm{mg}, 0.016 \mathrm{mmol})$. The reaction was sealed, protected from light and maintained for 24 hours before concentrating in vасио. Purification by column chromatography (99.5:0.5 hexanes/EtOAc) afforded 22 $\operatorname{mg}(88 \%)$ of amide 55a as a pale yellow oil: $[\square]^{25}=-1.6,[\square]^{25}{ }_{577}=-2.4,[\square]^{25}{ }_{546}=-1.4$, $[\square]_{435}^{25}=-1.9,[\square]_{405}^{25}=-3.0\left(\mathrm{c} 0.4, \mathrm{CHCl}_{3}\right) ; \mathrm{R}_{\mathrm{f}}=0.52\left(4: 1\right.$ hexanes-EtOAc); ${ }^{1} \mathrm{H} \mathrm{NMR}$ $\left(500 \mathrm{MHz}, \mathrm{CDCl}_{3}\right) \square 7.06(\operatorname{app~dd}, J=18.7,8.6 \mathrm{~Hz}, 2 \mathrm{H}), 6.88(\operatorname{app~t}, J=7.0 \mathrm{~Hz}, 2 \mathrm{H})$, 5.55 (ddd, $J=17.9,10.1,8.3 \mathrm{~Hz}, 1 \mathrm{H}), 5.26$ (d, $J=17.2 \mathrm{~Hz}, 1 \mathrm{H}), 5.19$ (dd, $J=10.2,0.6$ $\mathrm{Hz}, 1 \mathrm{H}), 5.02$ (q, $J=7.6 \mathrm{~Hz}, 1 \mathrm{H}), 3.82$ (s, 3H), 1.54-1.64 (m, 1H), 1.39-1.48 (m, 1H), 1.37 (sextet, $J=7.3 \mathrm{~Hz}, 2 \mathrm{H}), 0.93(\mathrm{t}, J=7.2 \mathrm{~Hz}, 3 \mathrm{H}) ;{ }^{13} \mathrm{C} \mathrm{NMR}\left(125 \mathrm{MHz}, \mathrm{CDCl}_{3}\right) \square$ $159.5,156.5\left(\mathrm{q}, J_{\text {C-C-F }}=34 \mathrm{~Hz}\right), 135.2,131.6,130.8,127.7,119.0,116.3\left(\mathrm{q}, J_{\mathrm{C}-\mathrm{F}}=286\right.$ Hz), 113.6, 113.5, 60.6, 55.4, 33.8, 19.6, 13.9; IR (neat) 3083, 2964, 2877, 1690, 1511, 1299, 1250, 1202, 1187, 1148, $1034 \mathrm{~cm}^{-1}$; LRMS (ES) $m / z 324.10(\mathrm{M}+\mathrm{Na})^{+}$. Anal. Calcd for $\mathrm{C}_{15} \mathrm{H}_{18} \mathrm{~F}_{3} \mathrm{NO}_{2}$ : C, 59.79; H, 6.02; N, 4.65. Found: C, 60.05; H, 6.10; N, 4.69. 
Supporting Information Anderson, Donde, Douglas and Overman

B. 1H and 13C NMR Spectra of New Compounds

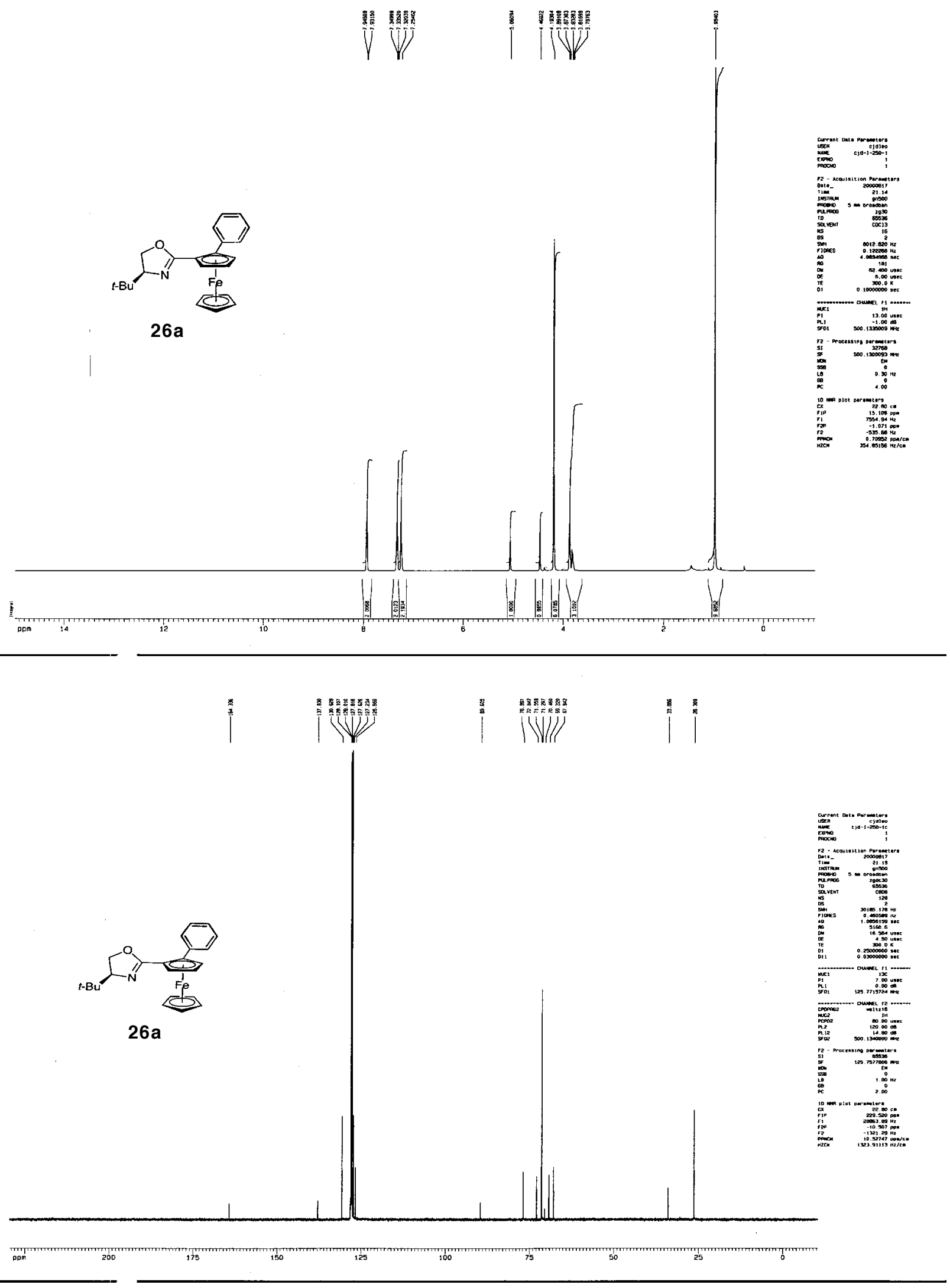


Supporting Information Anderson, Donde, Douglas and Overman

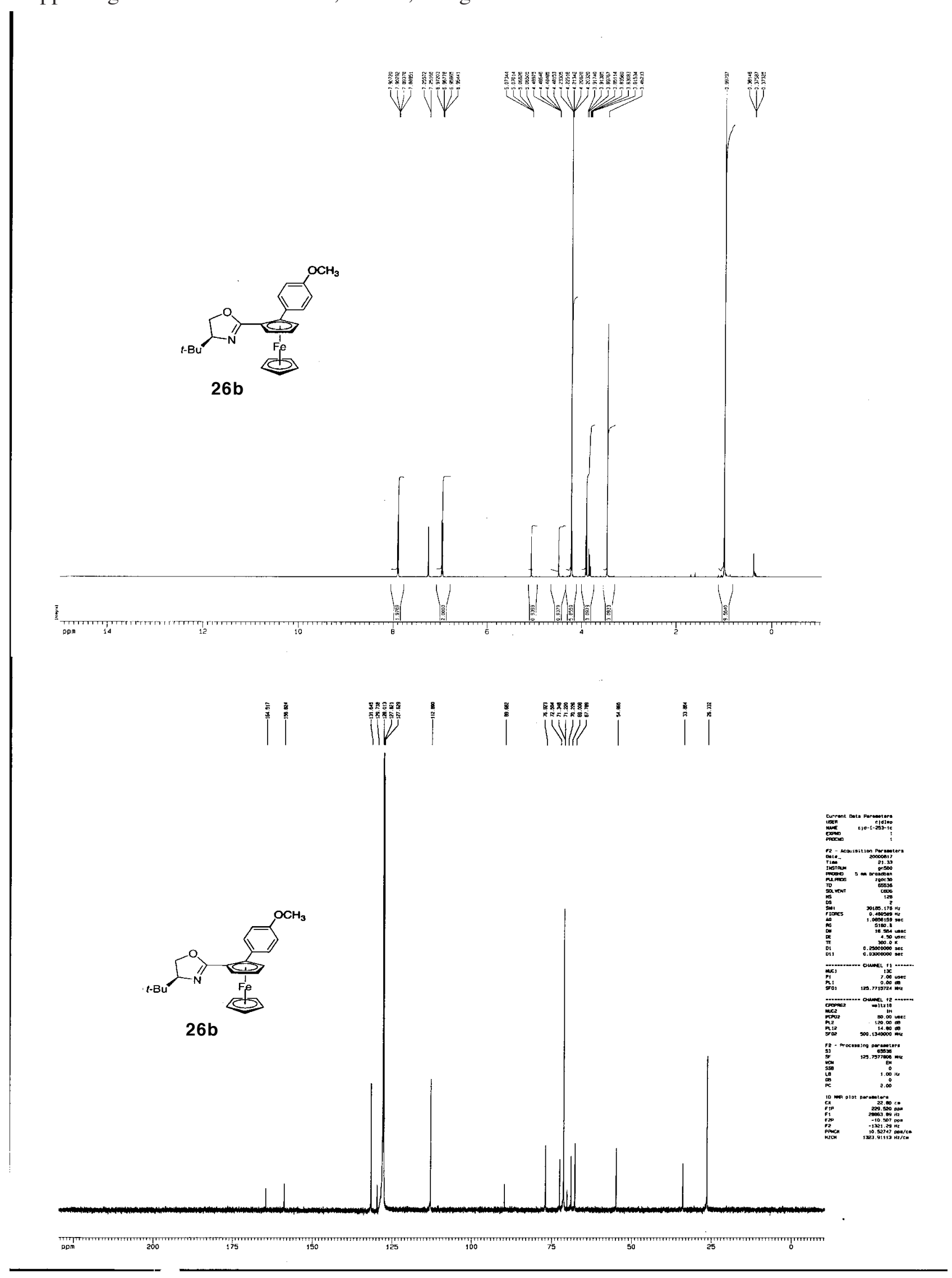


Supporting Information Anderson, Donde, Douglas and Overman
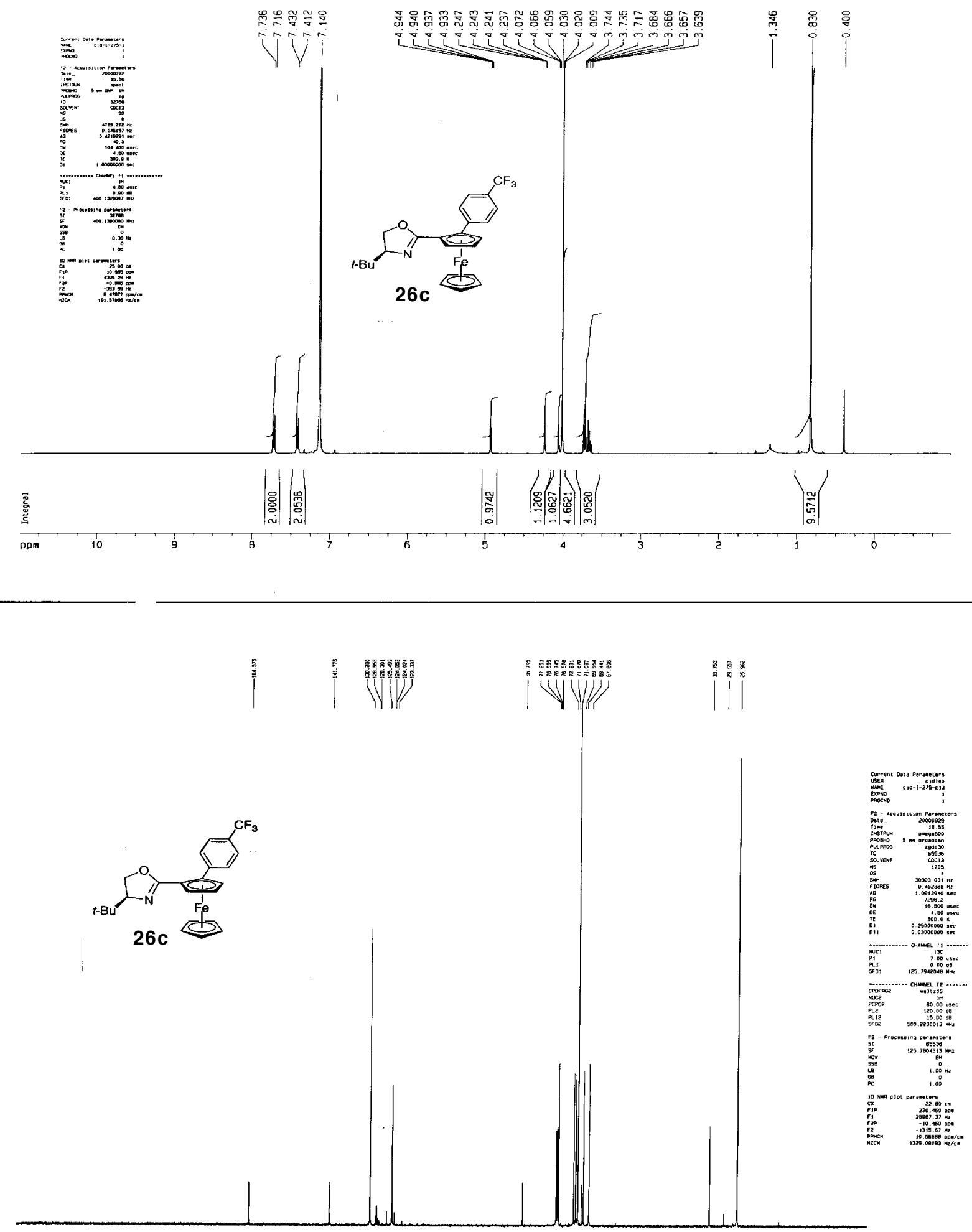

ppm 
Supporting Information Anderson, Donde, Douglas and Overman
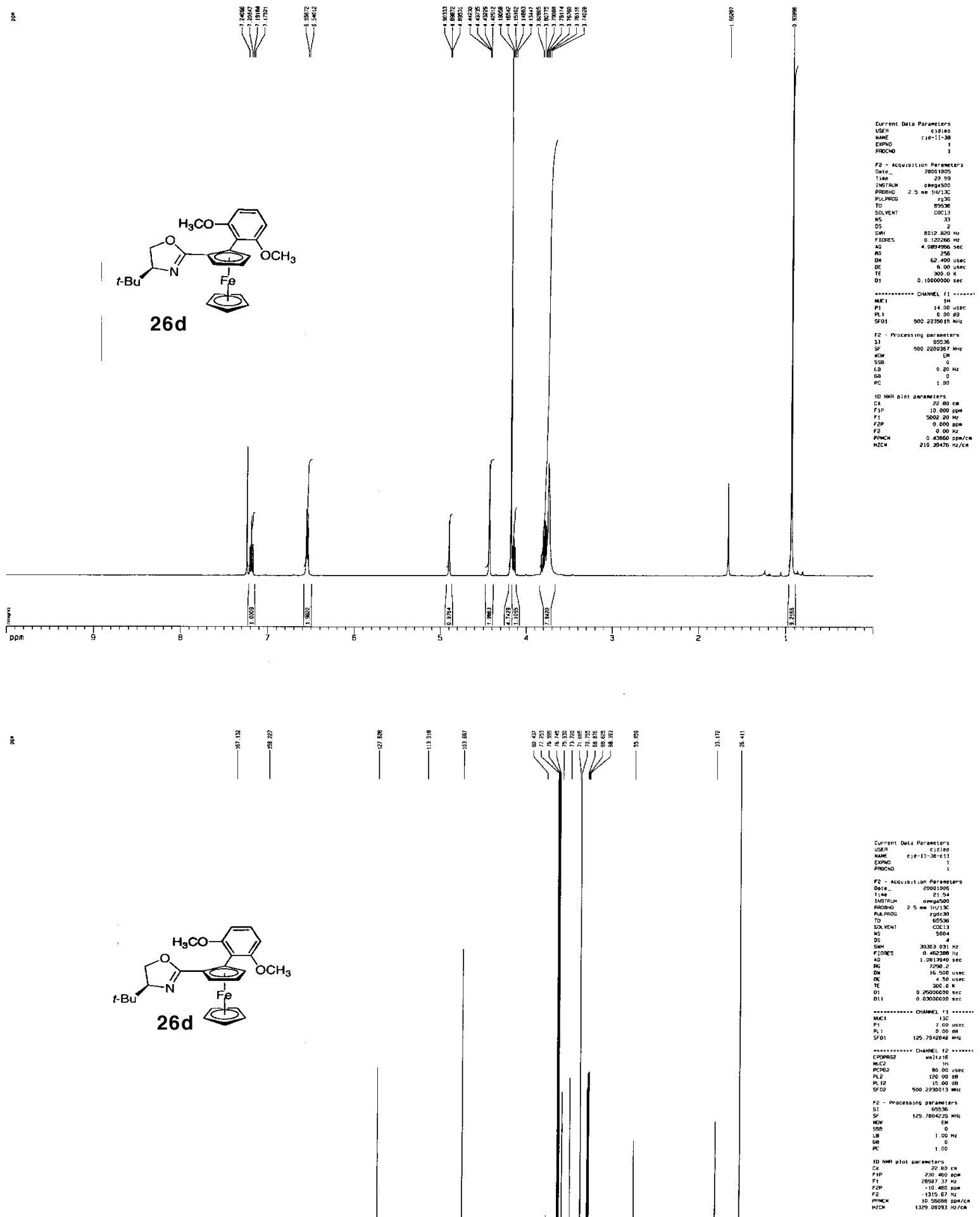
Supporting Information Anderson, Donde, Douglas and Overman

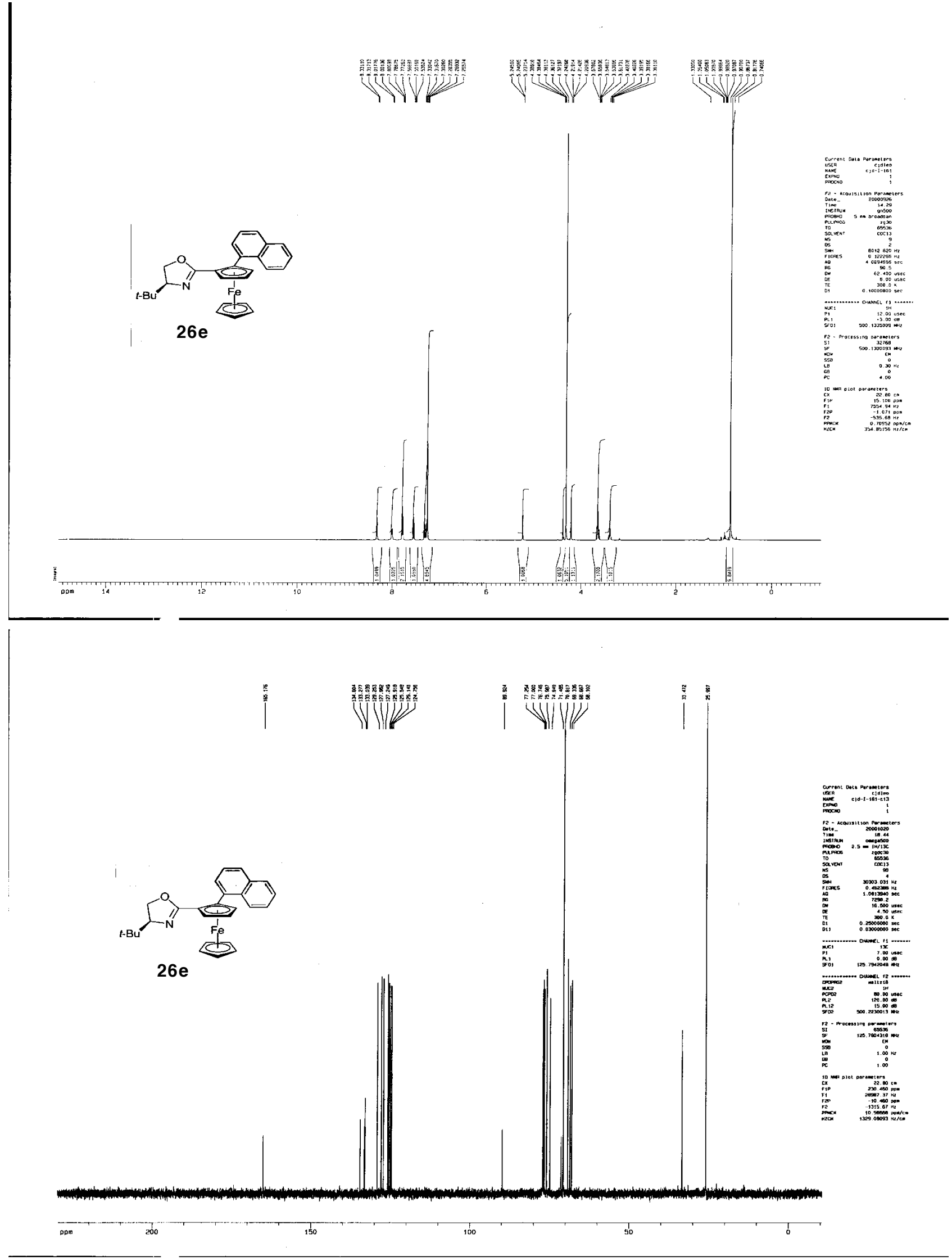


Supporting Information Anderson, Donde, Douglas and Overman
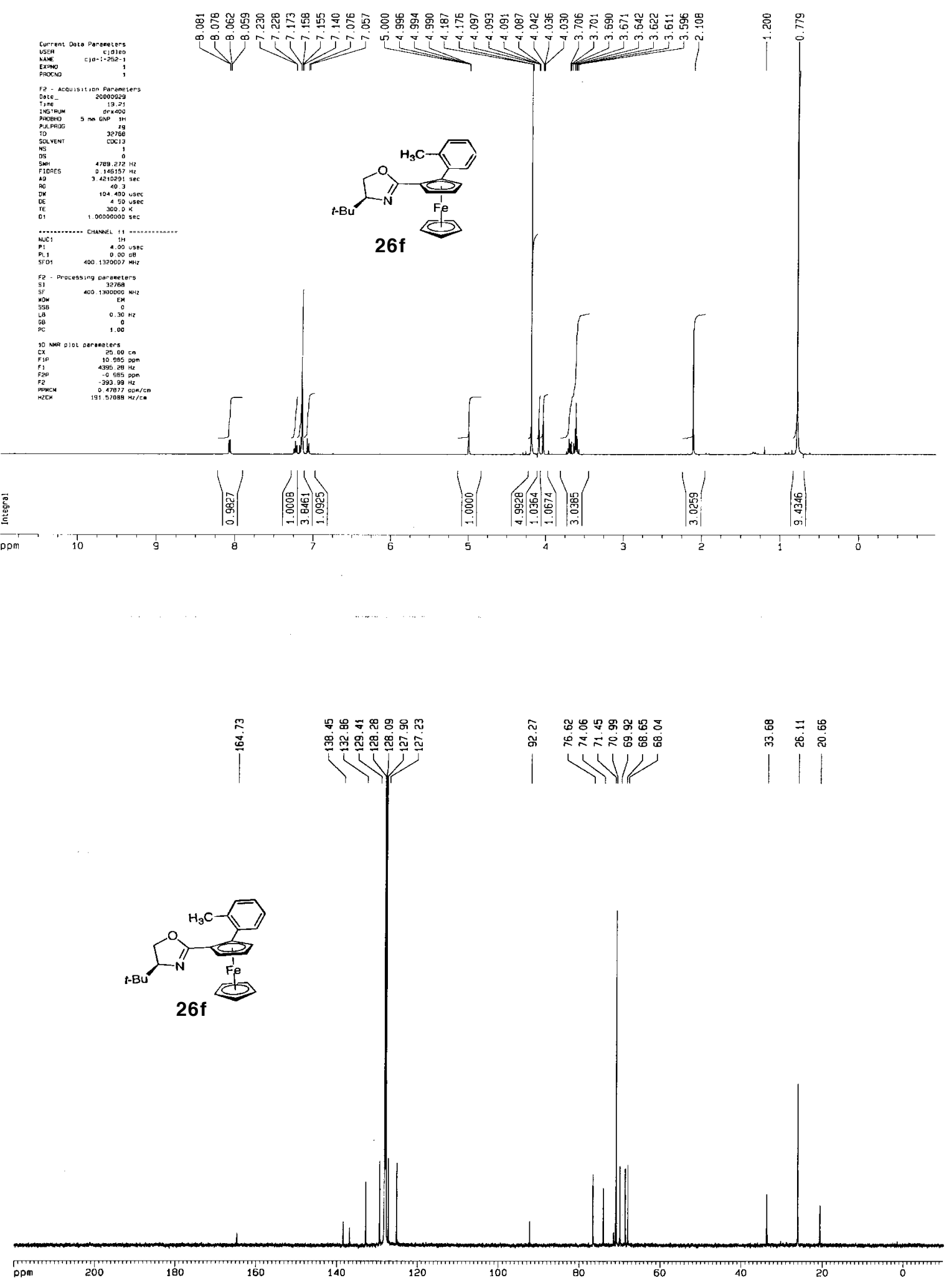
Supporting Information Anderson, Donde, Douglas and Overman
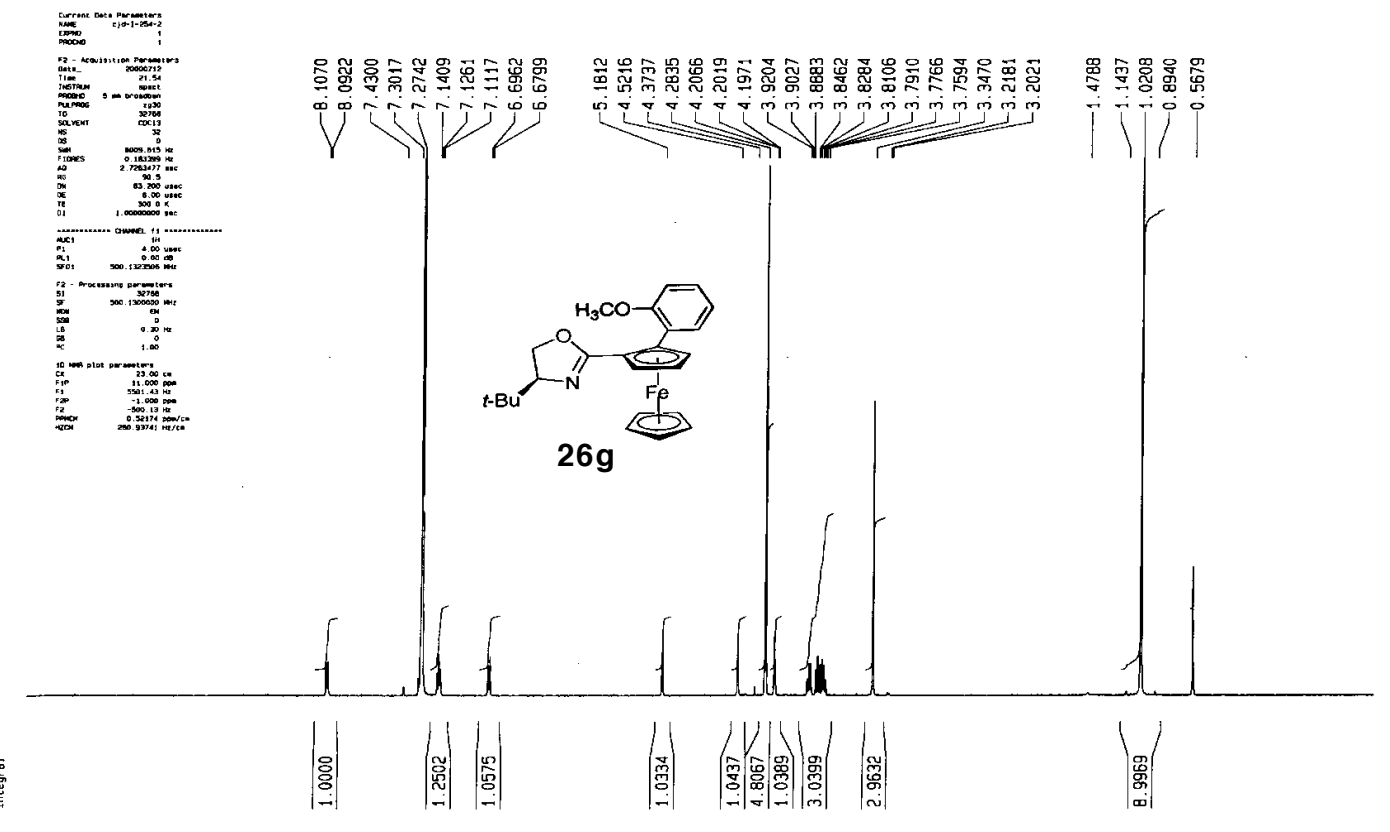

$\overline{\mathrm{E}}$
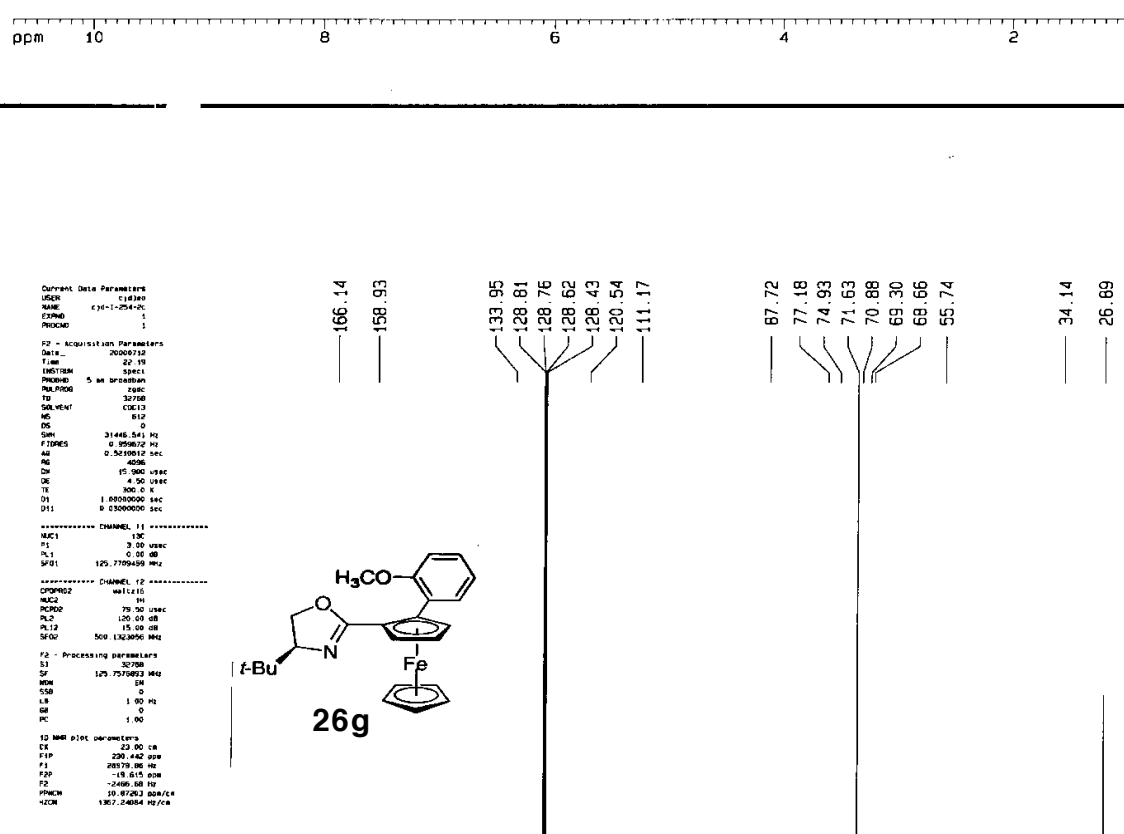

离

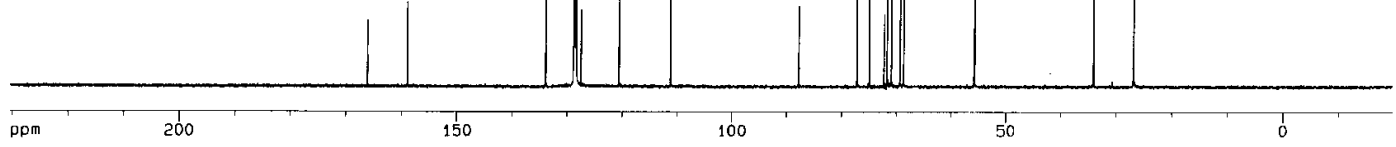


Supporting Information Anderson, Donde, Douglas and Overman

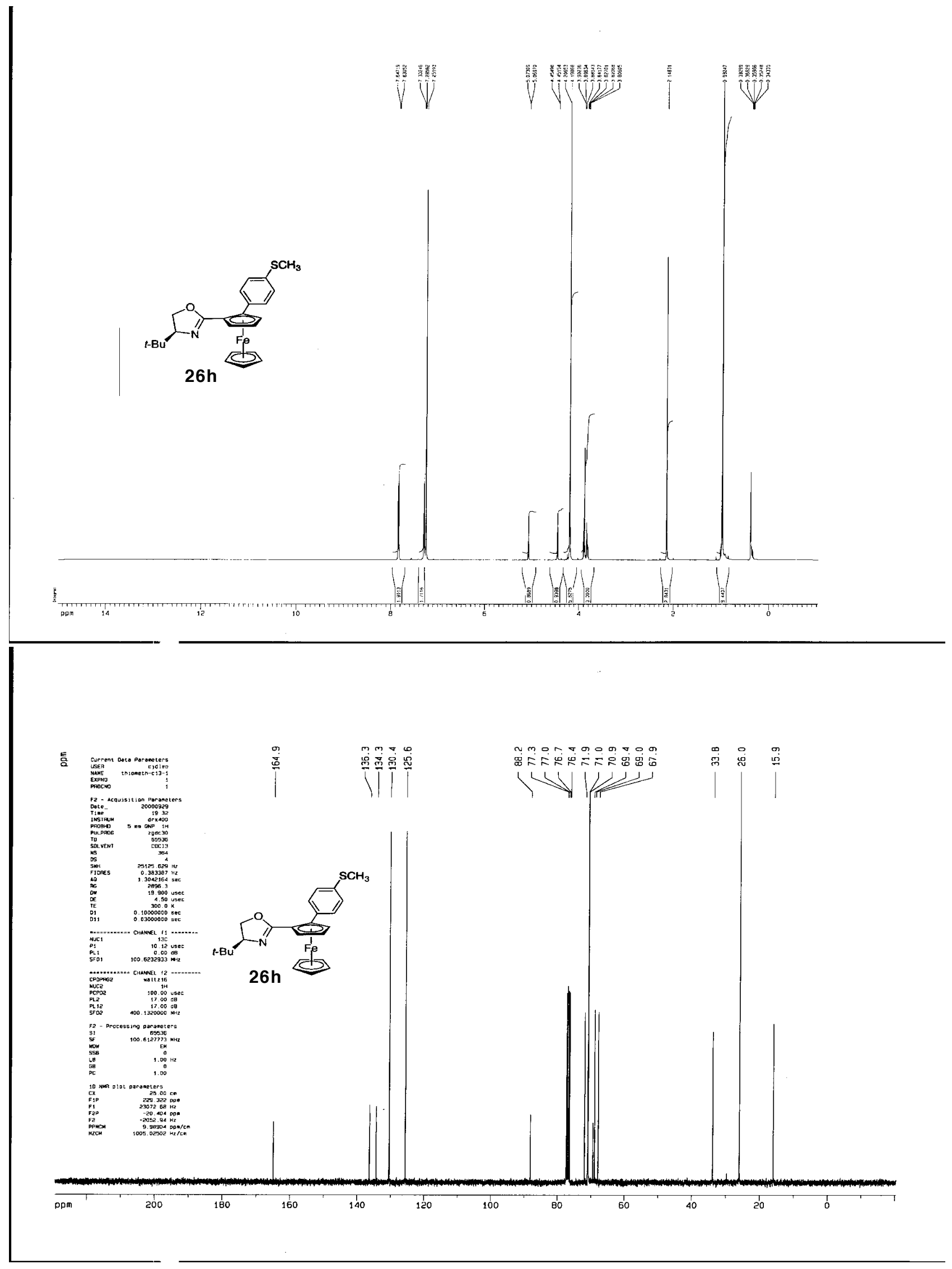




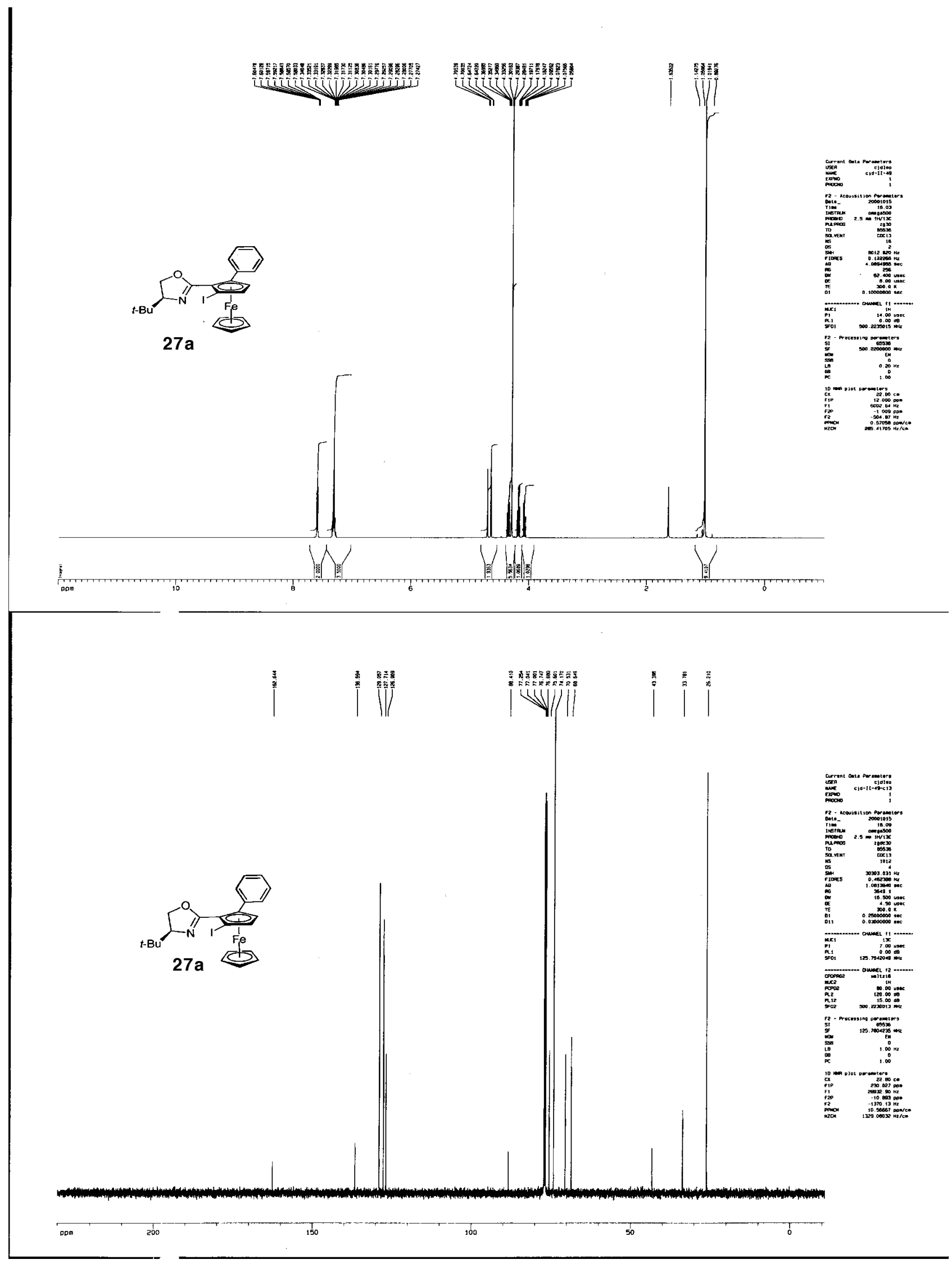


Supporting Information Anderson, Donde, Douglas and Overman

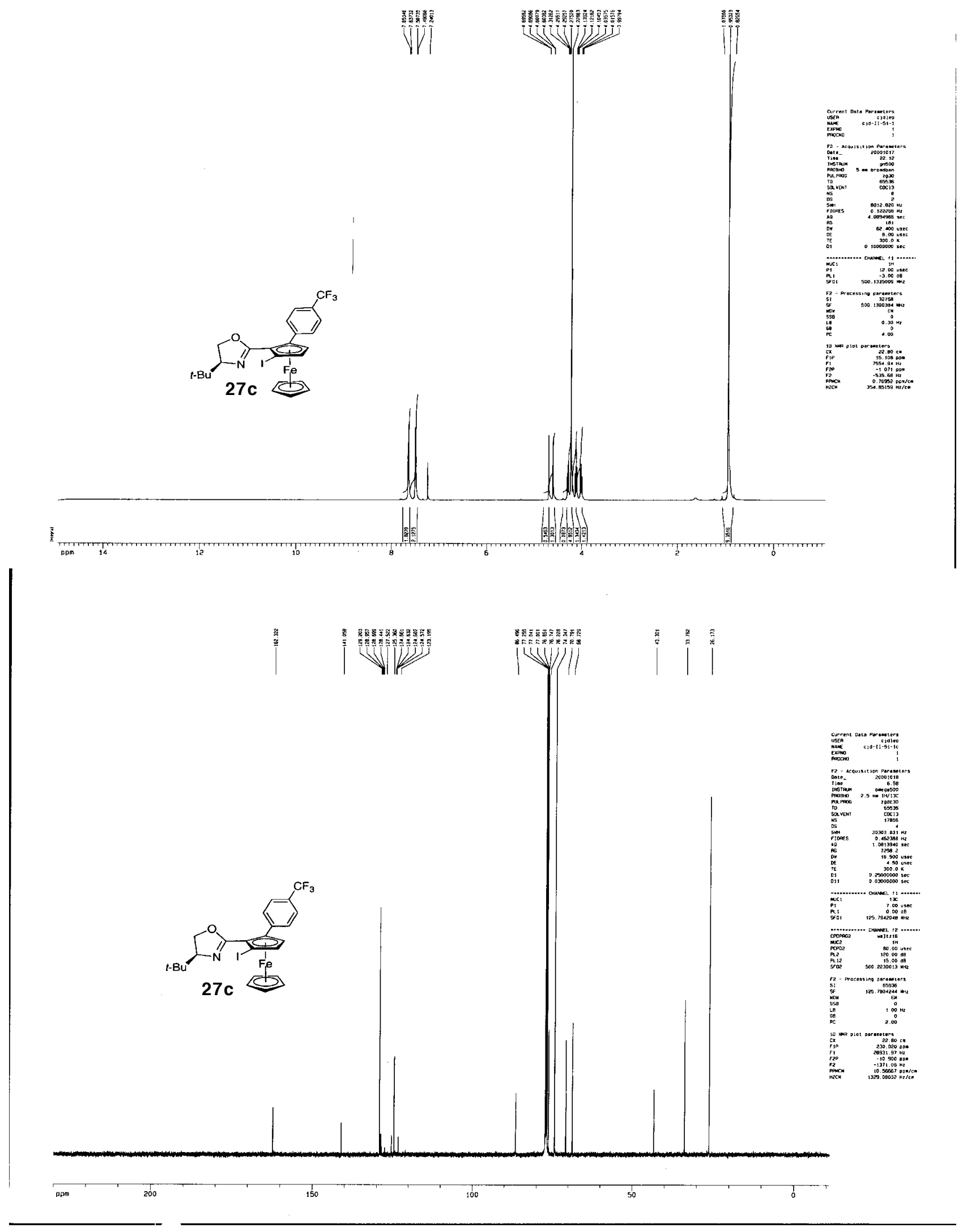




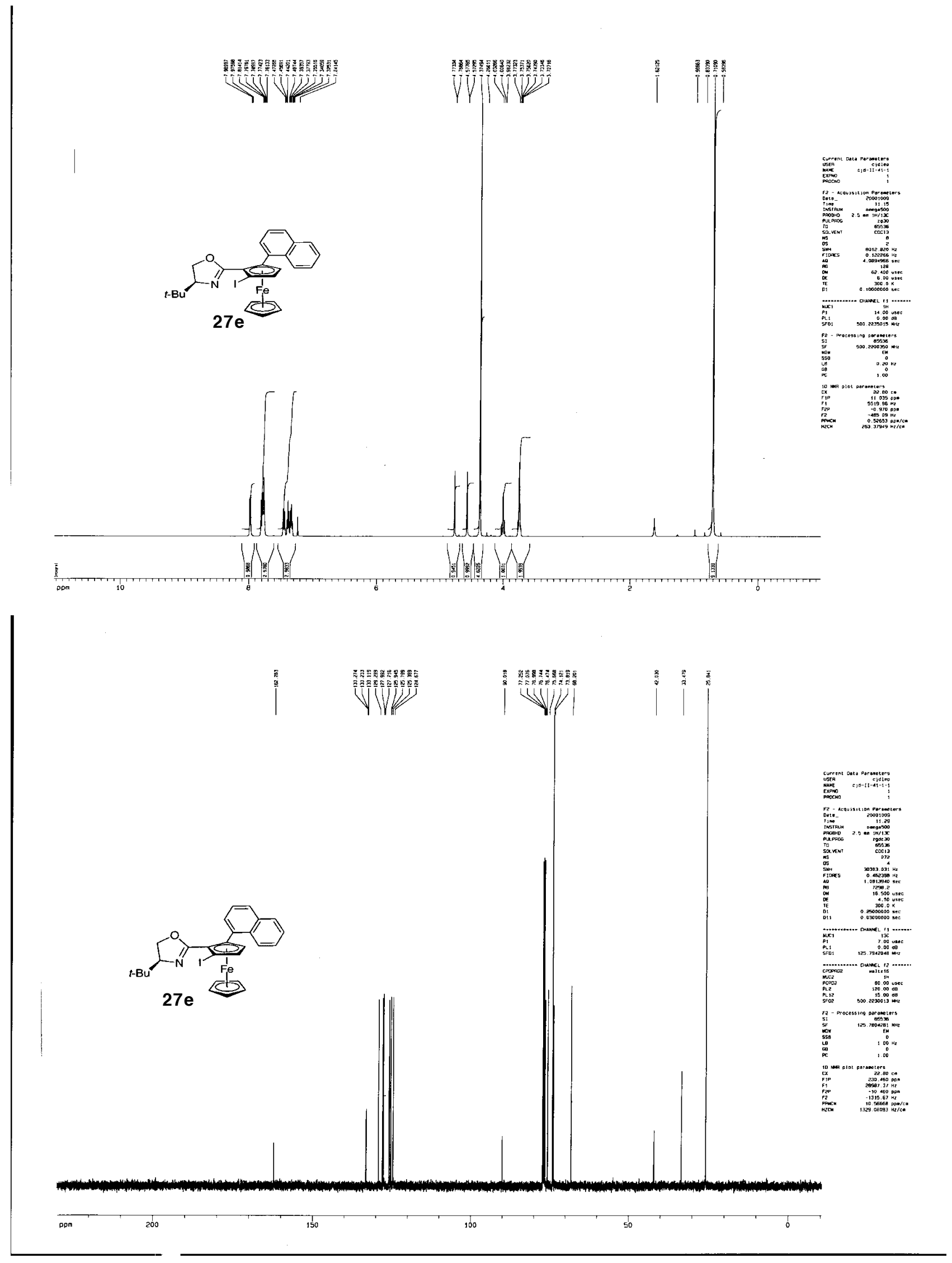


Supporting Information Anderson, Donde, Douglas and Overman
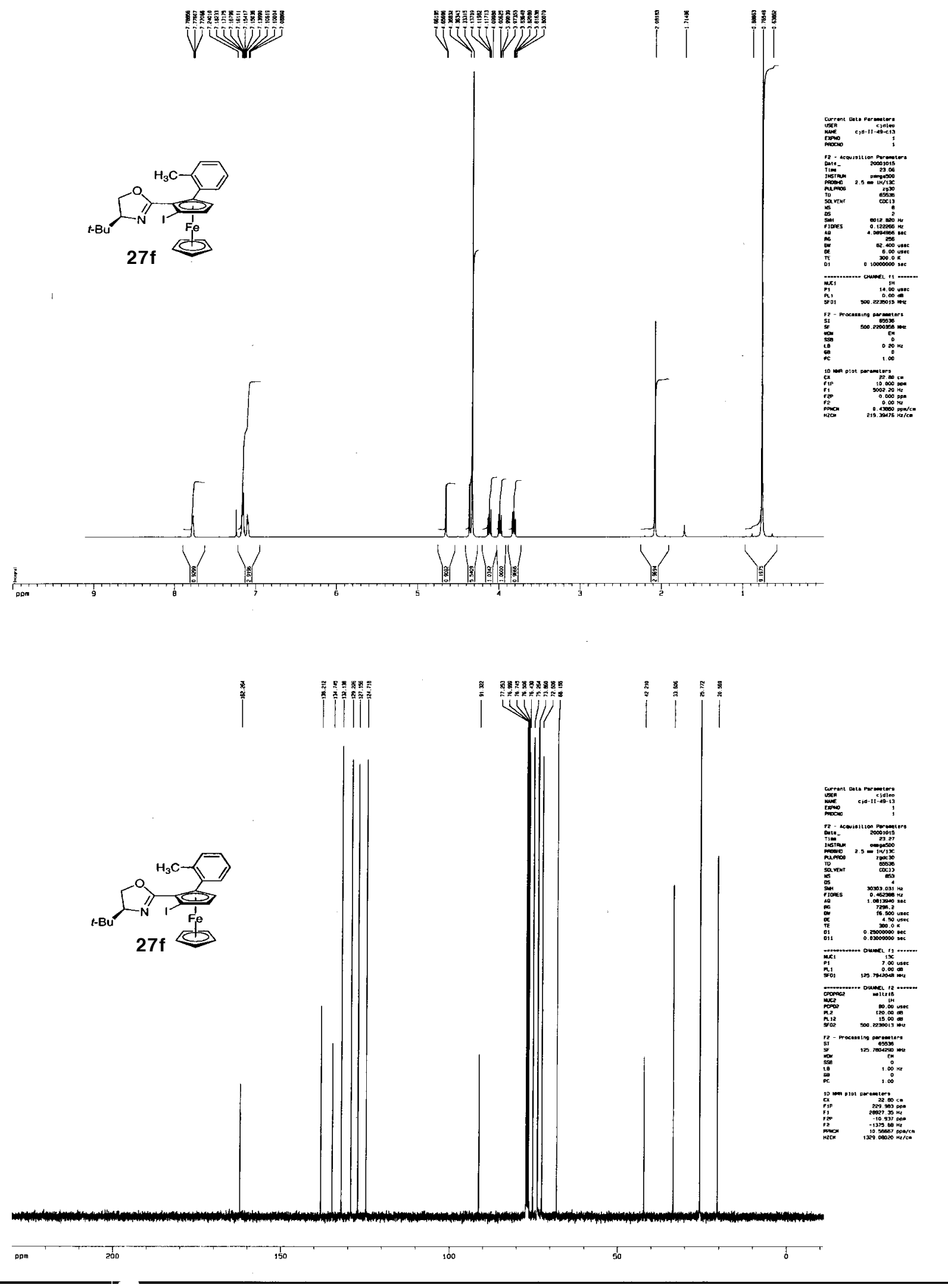

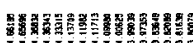

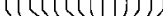

yre

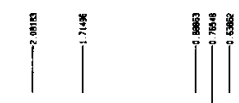


Supporting Information Anderson, Donde, Douglas and Overman

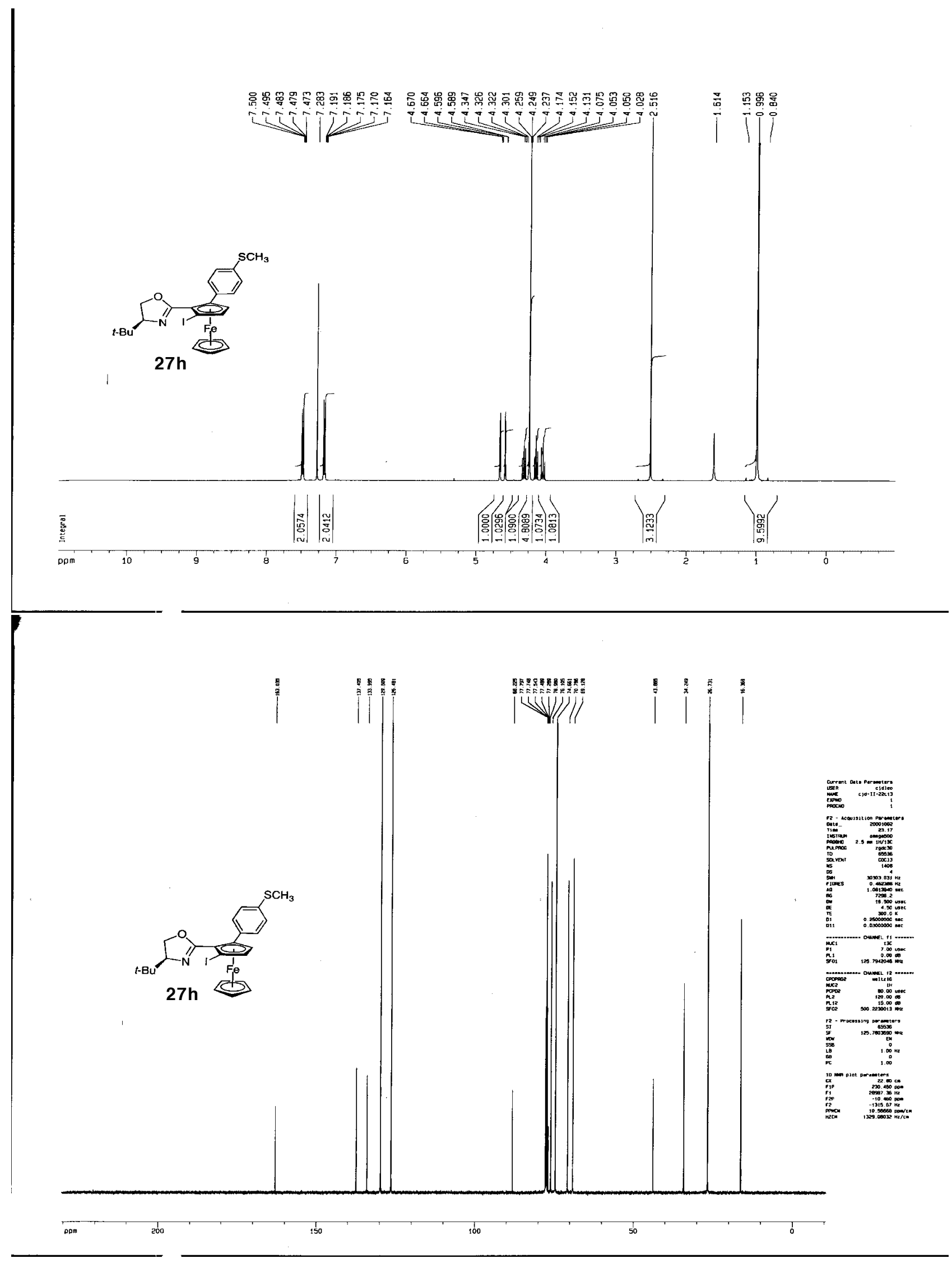


Supporting Information Anderson, Donde, Douglas and Overman

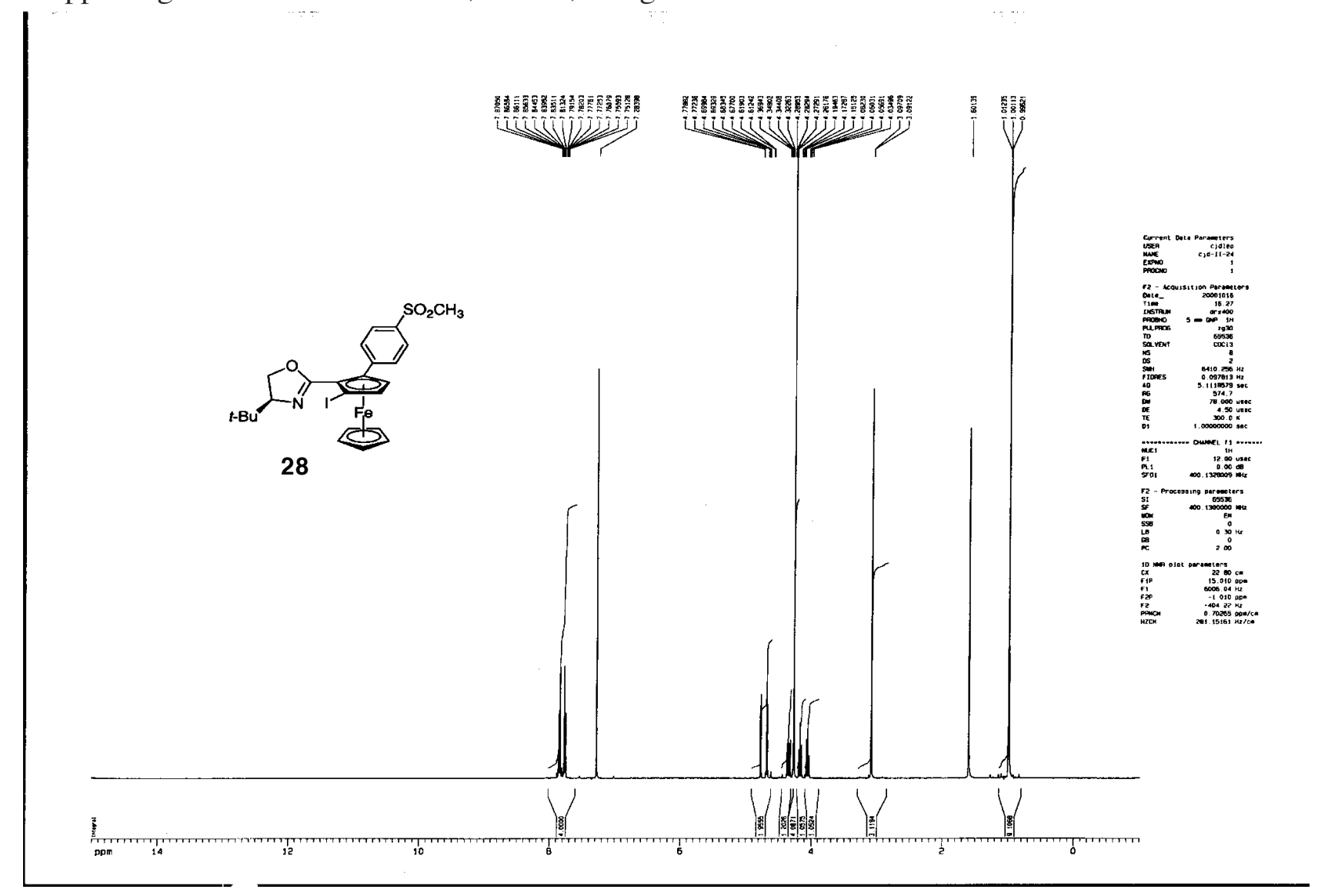



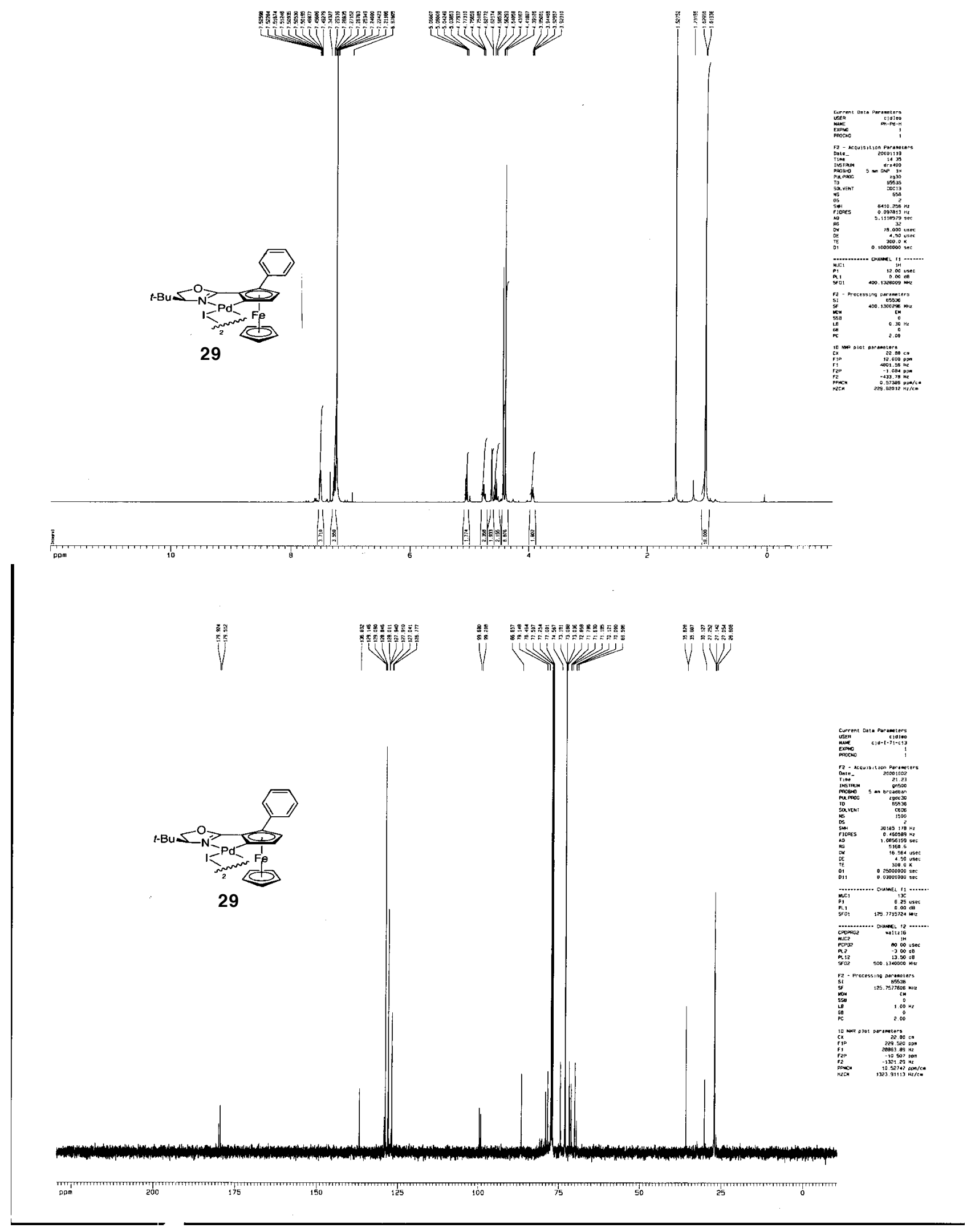
Supporting Information Anderson, Donde, Douglas and Overman

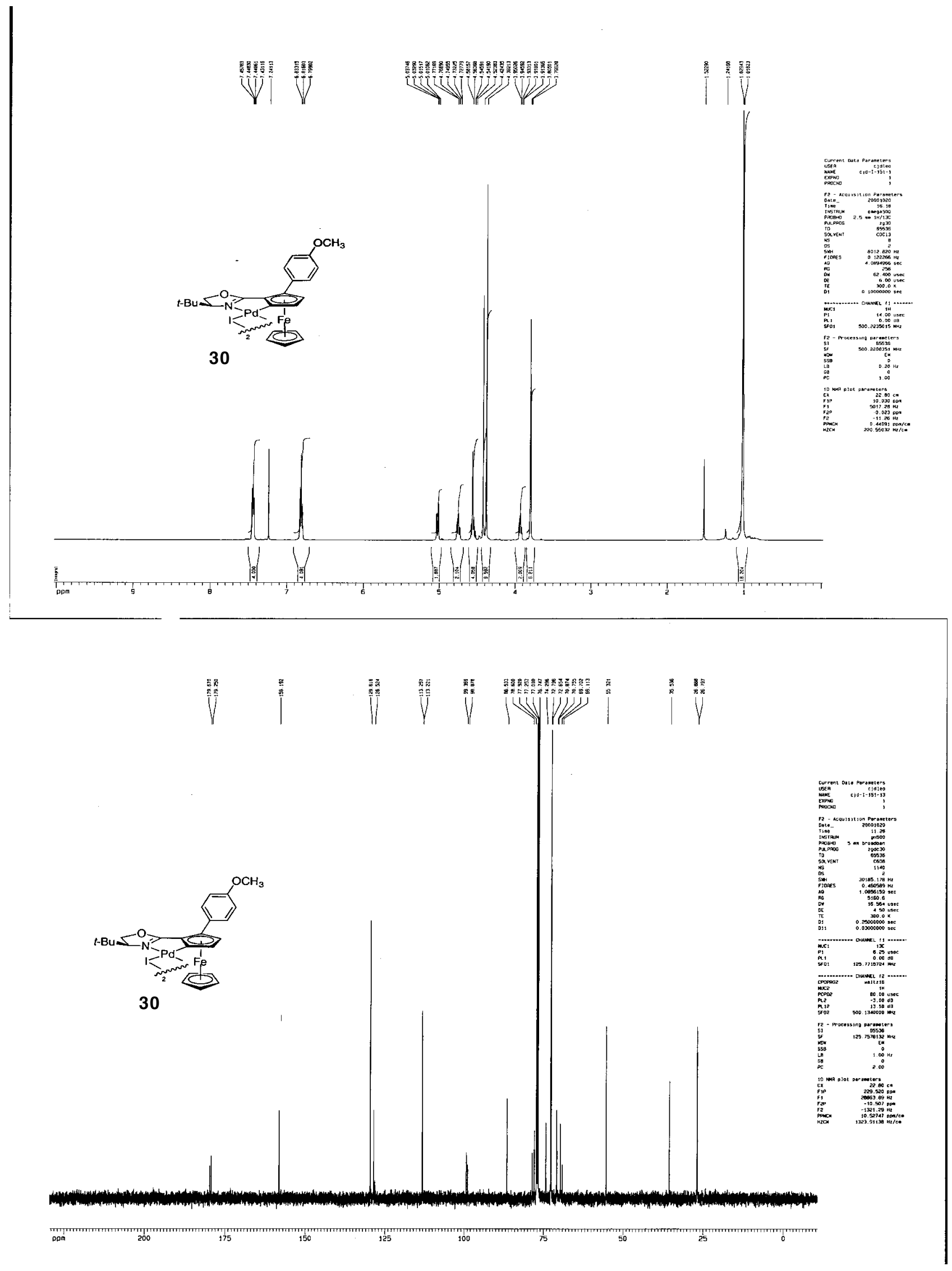




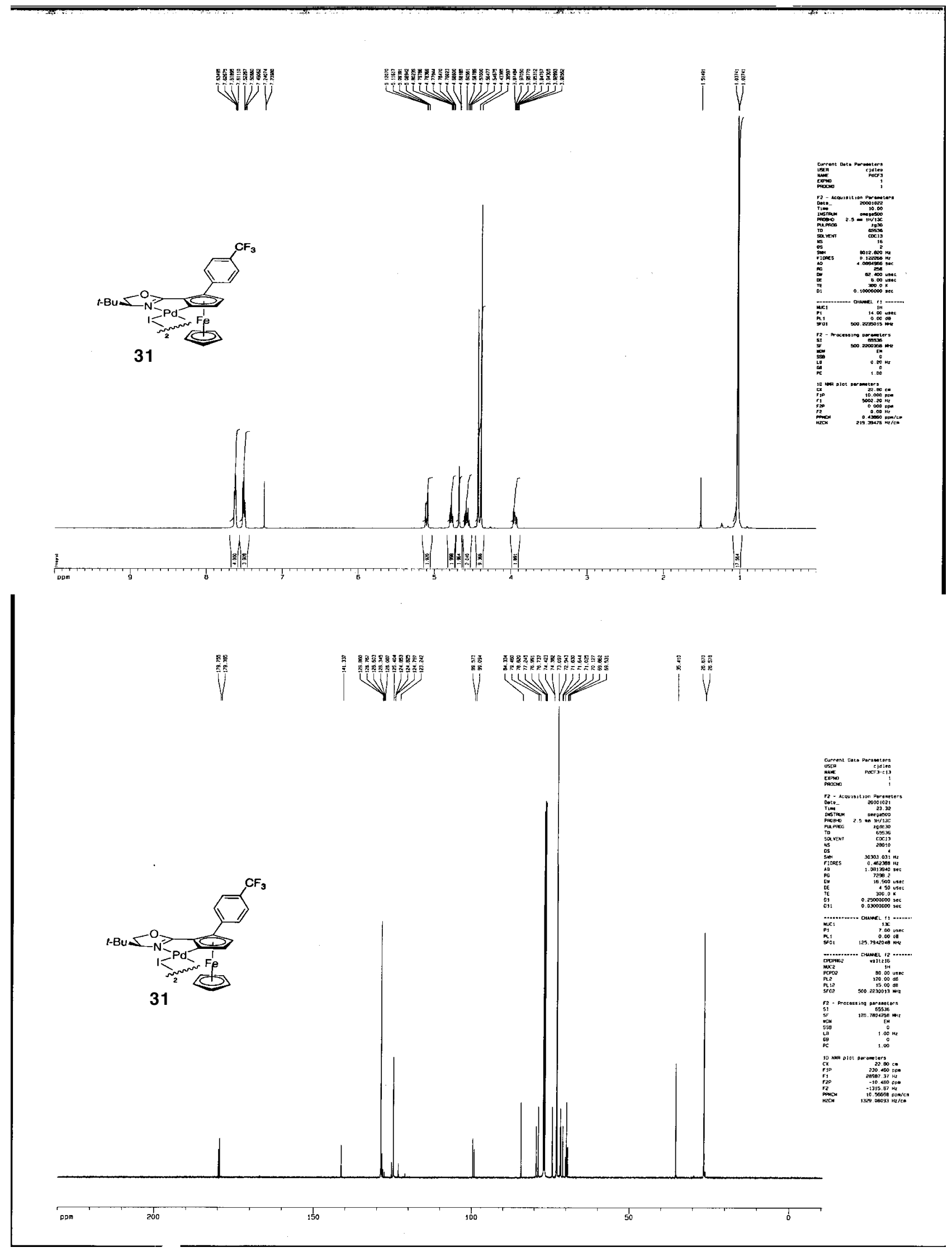


Supporting Information Anderson, Donde, Douglas and Overman

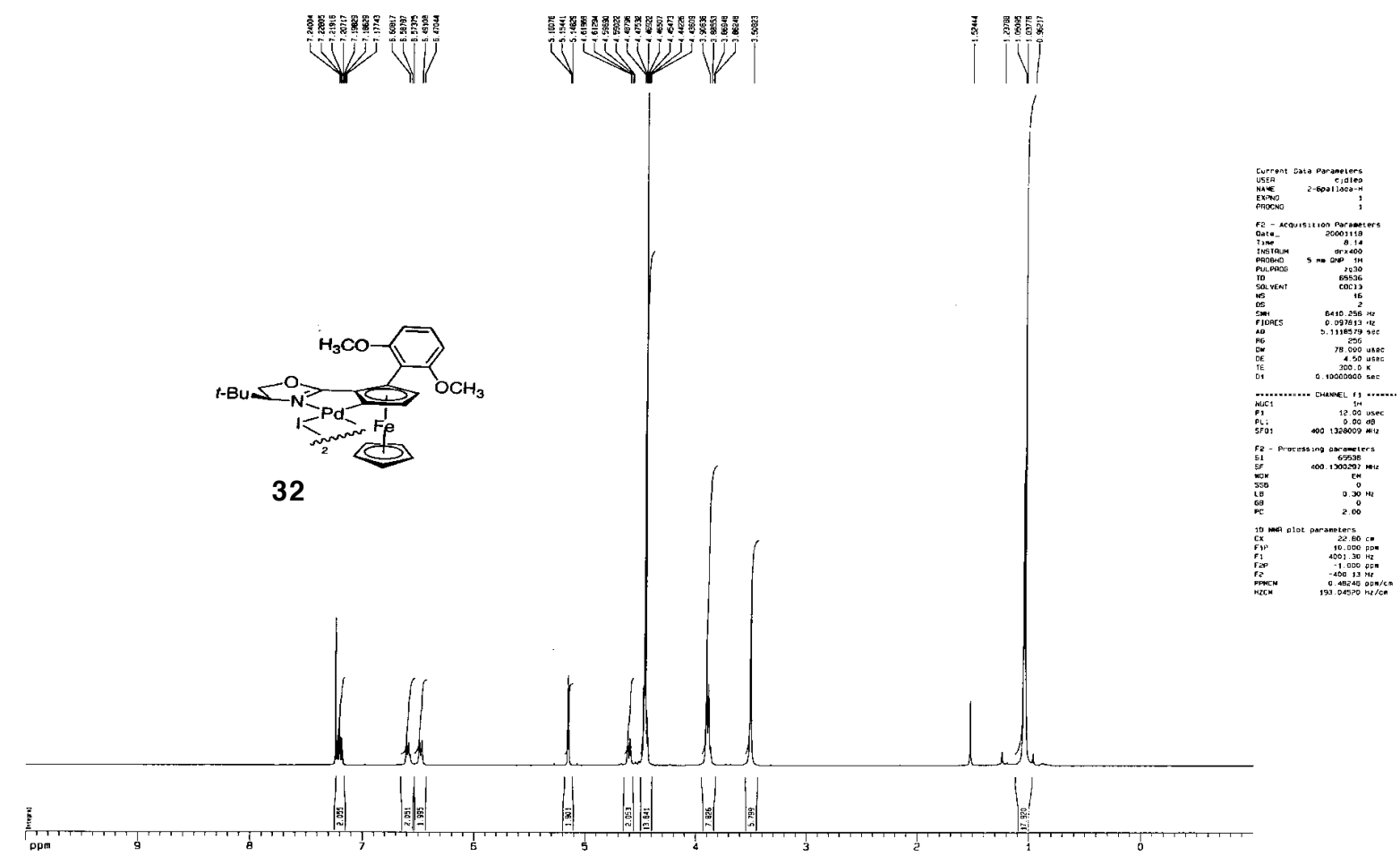

13C spectrum with $1 \mathrm{H}$ decoupling

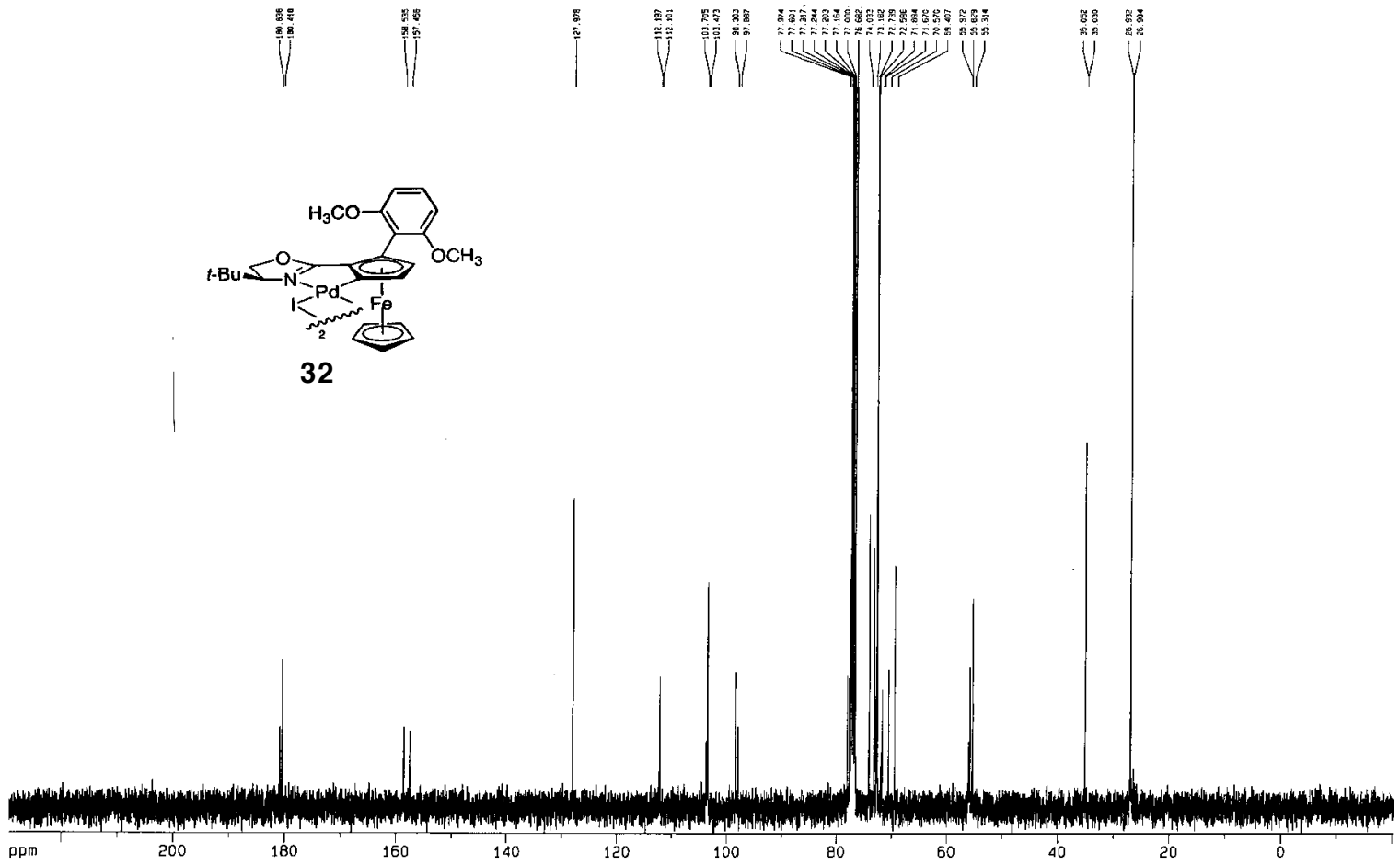




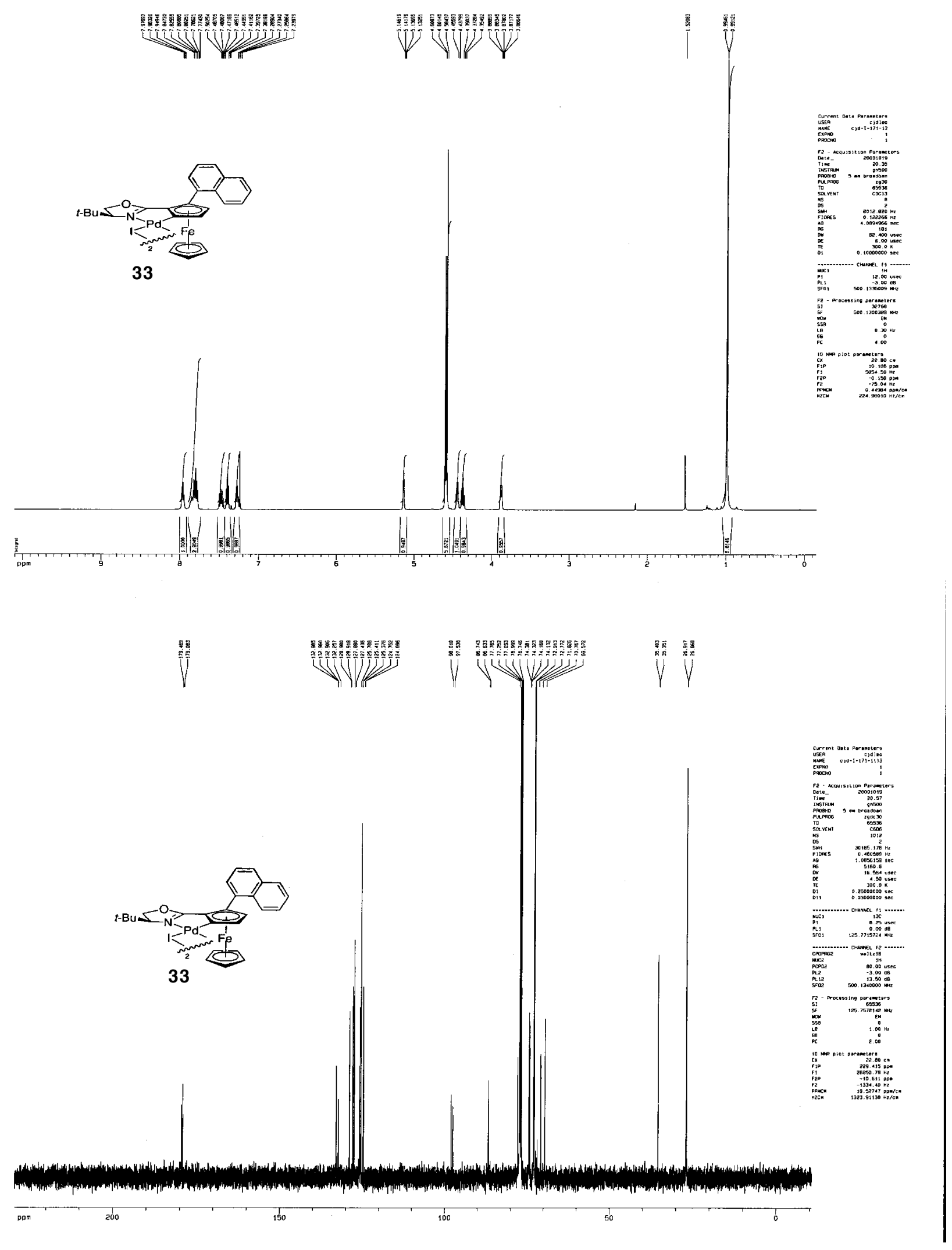




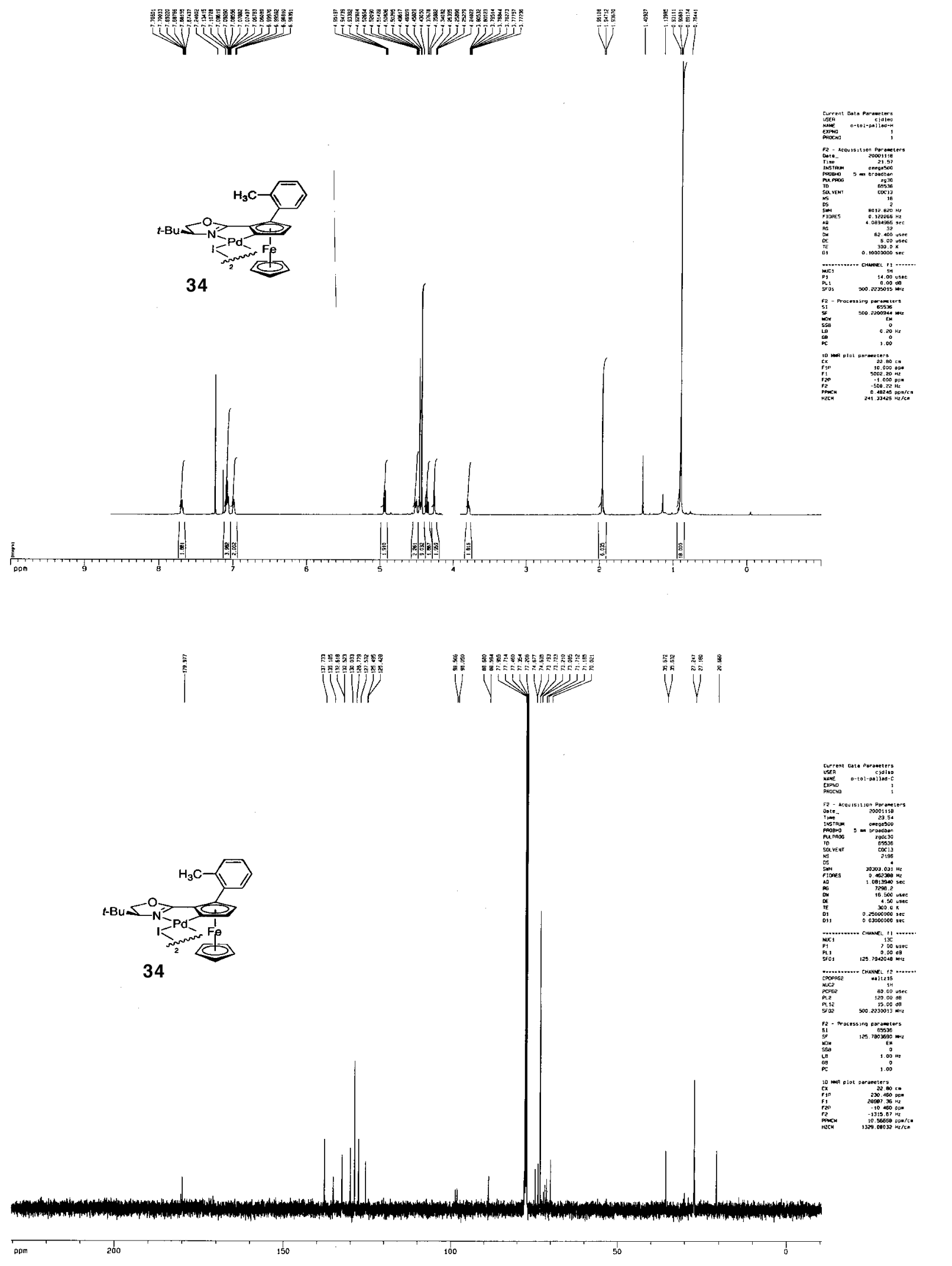




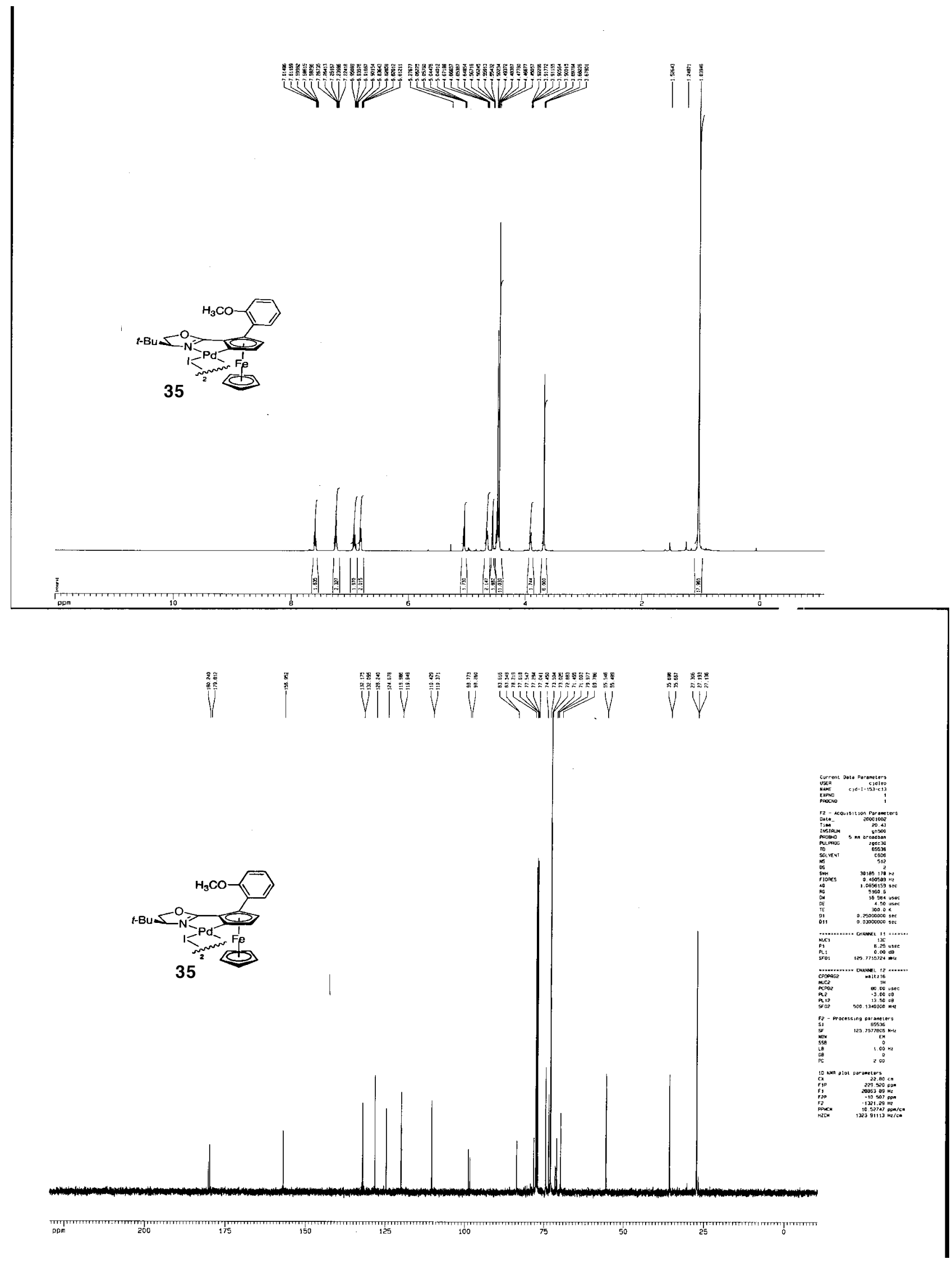


Supporting Information Anderson, Donde, Douglas and Overman

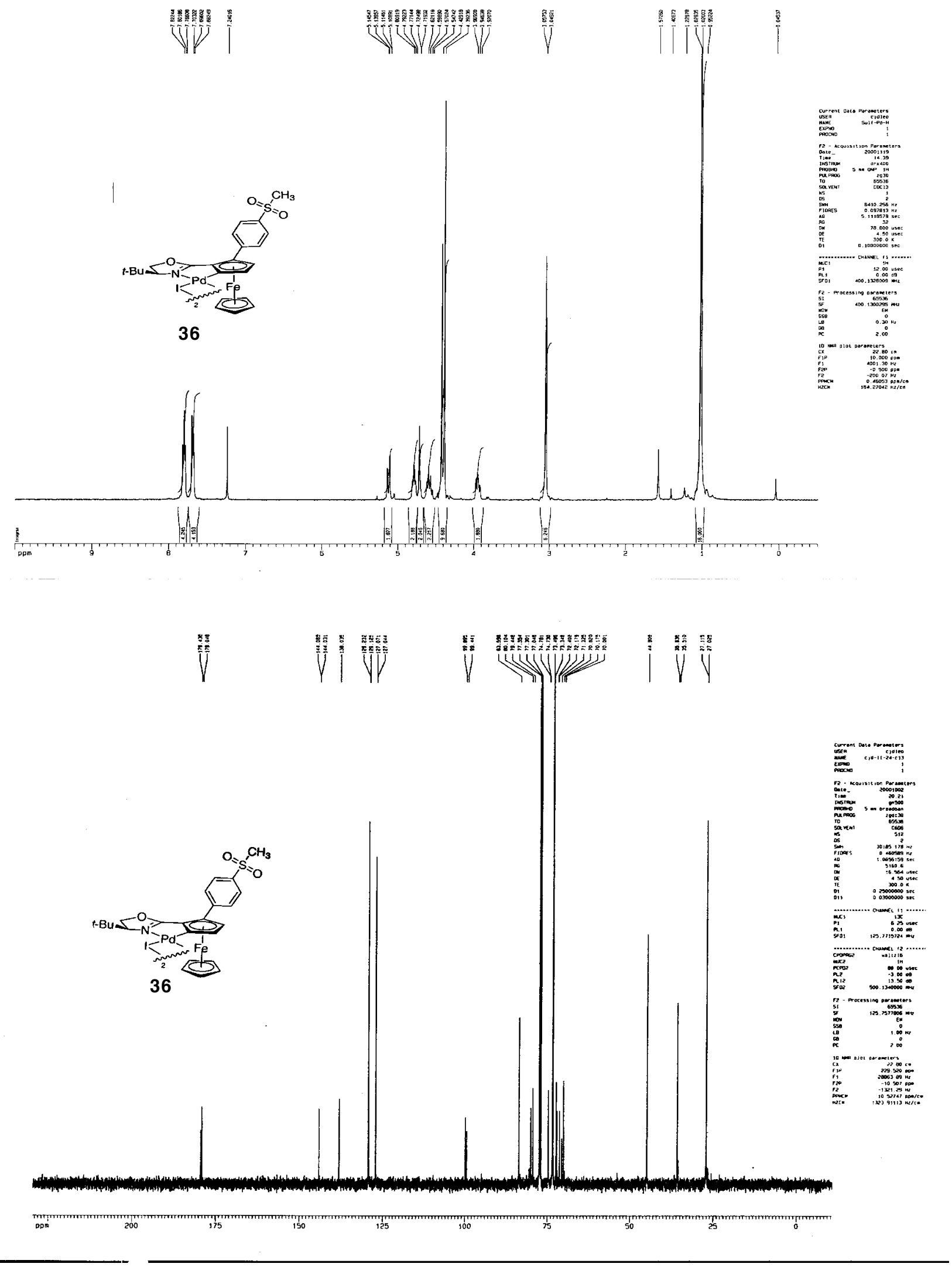



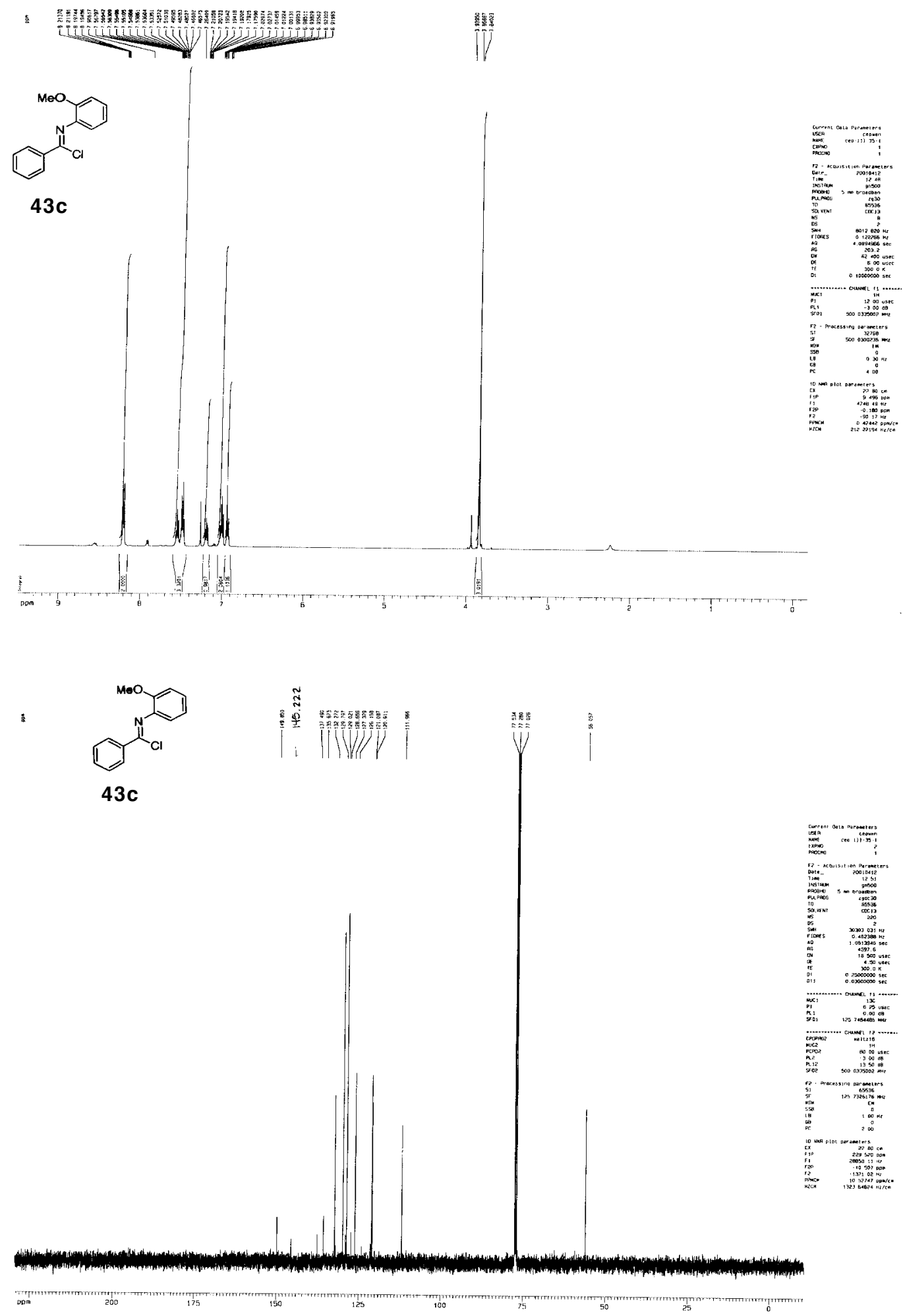


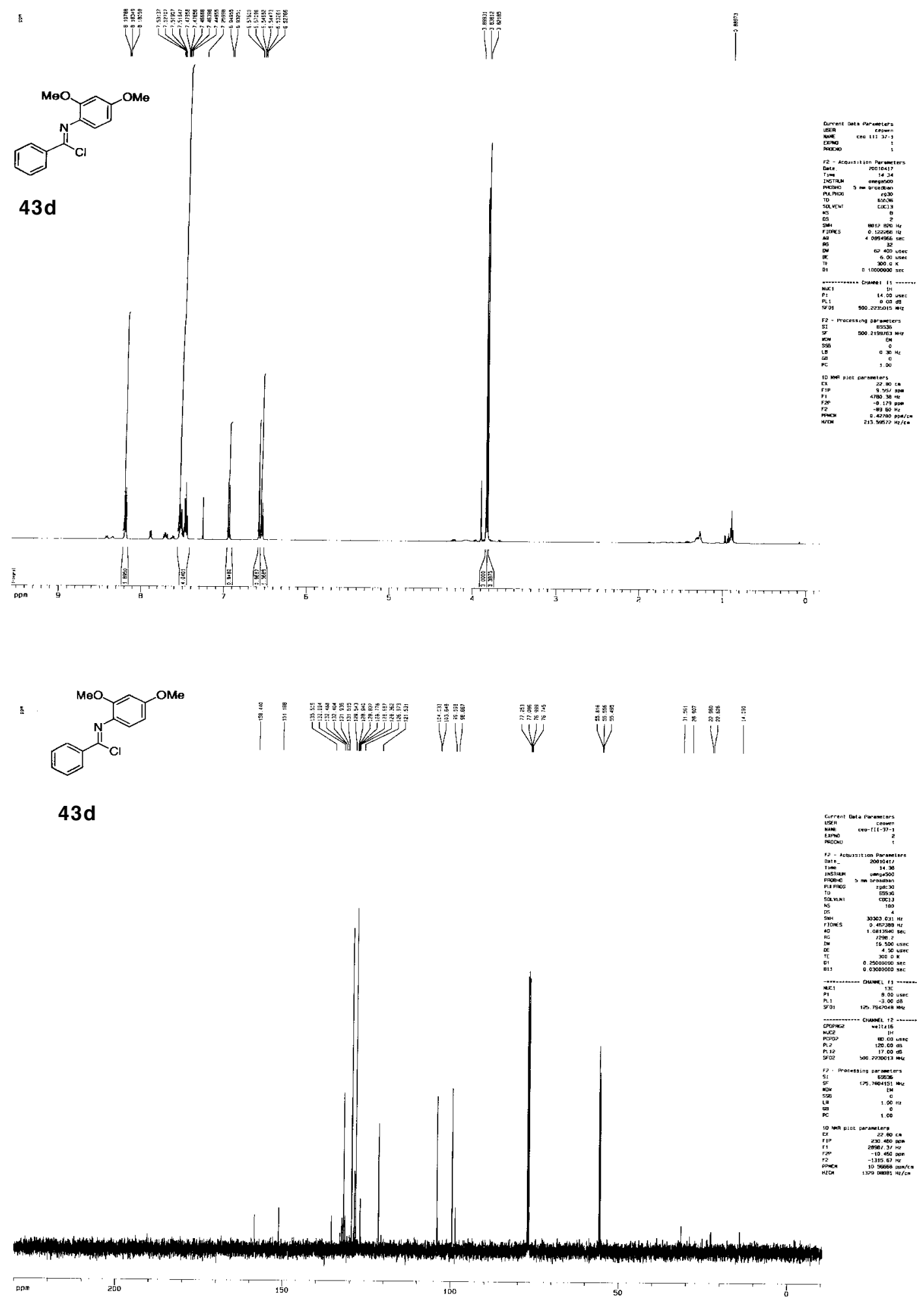



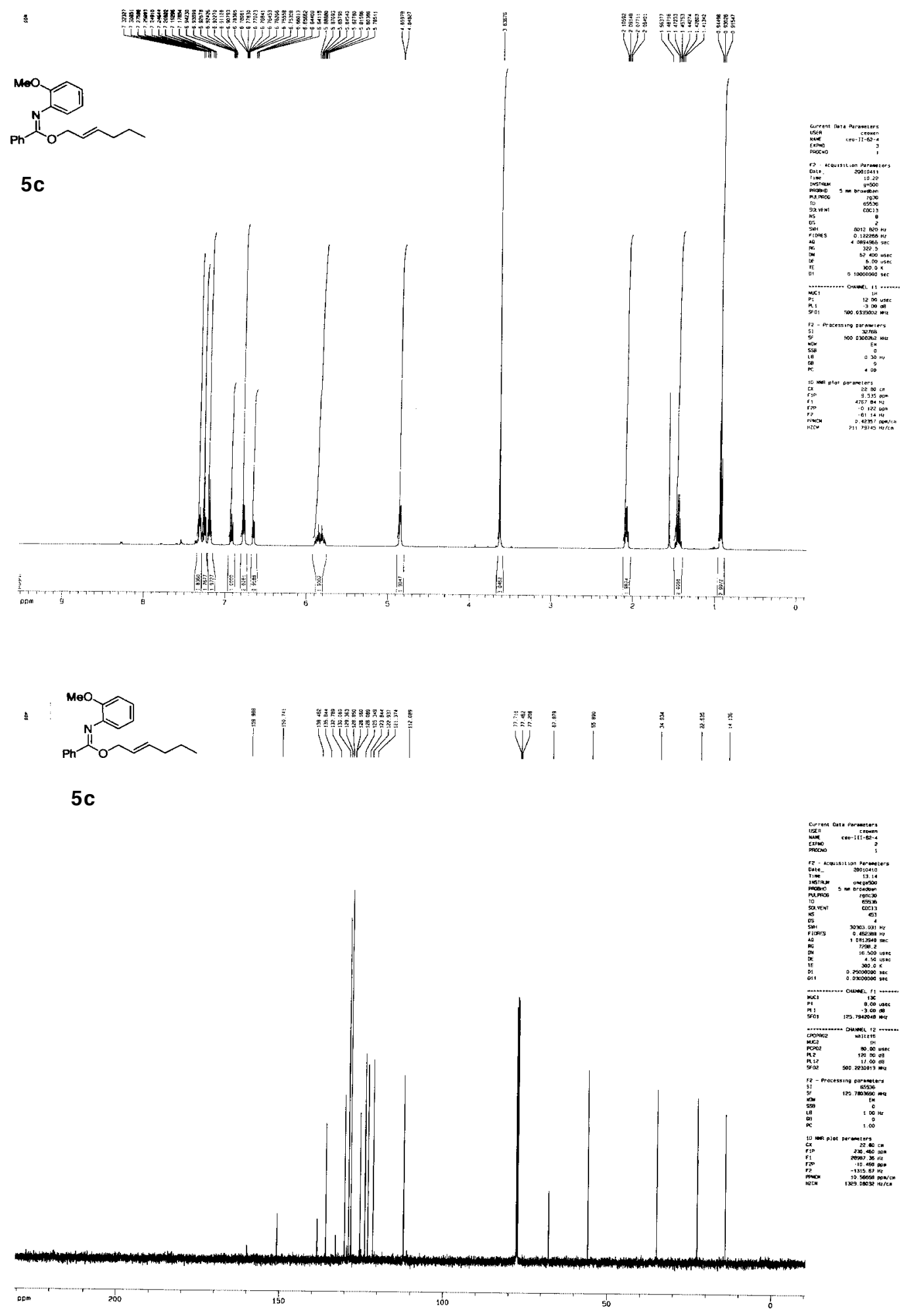

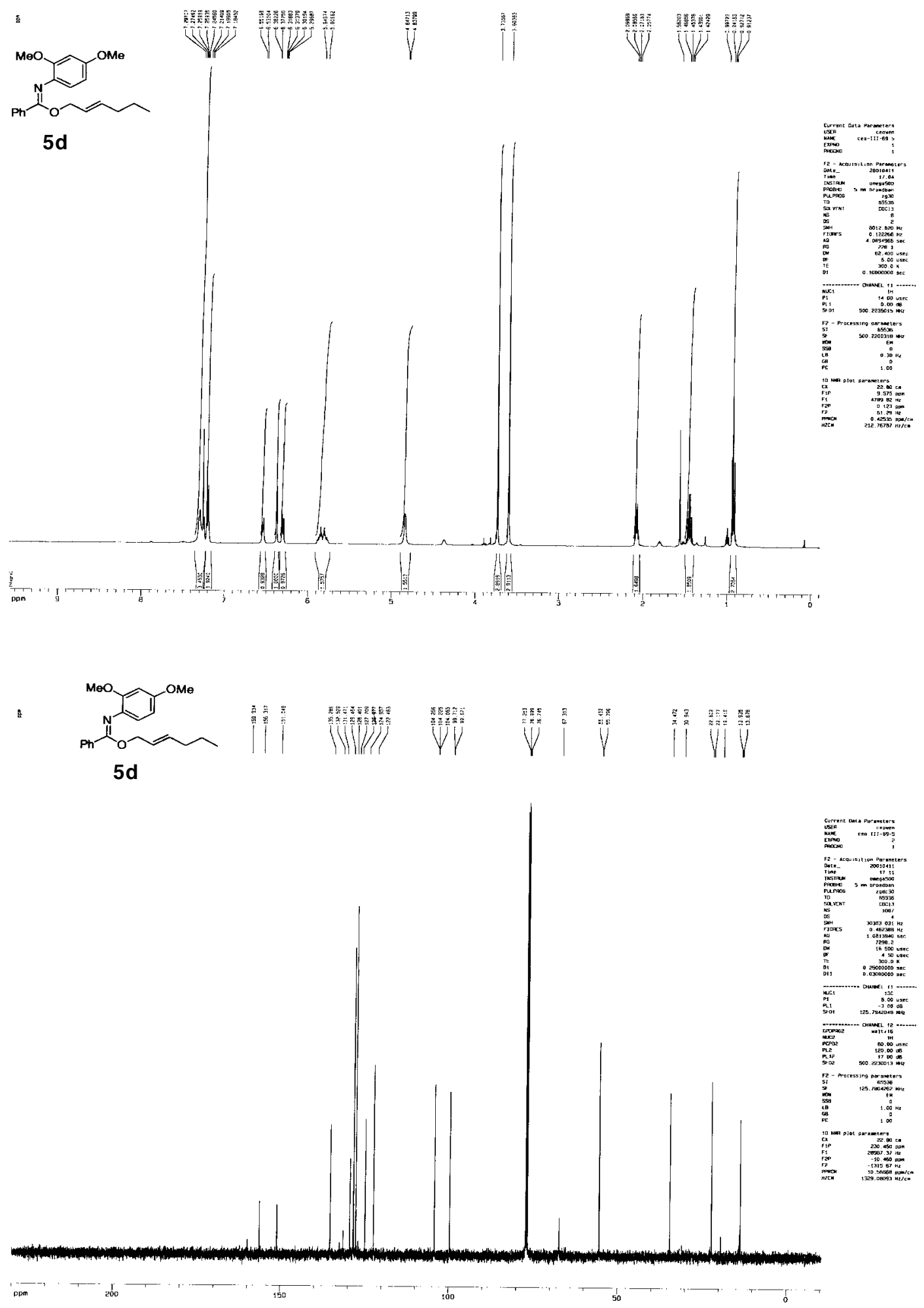

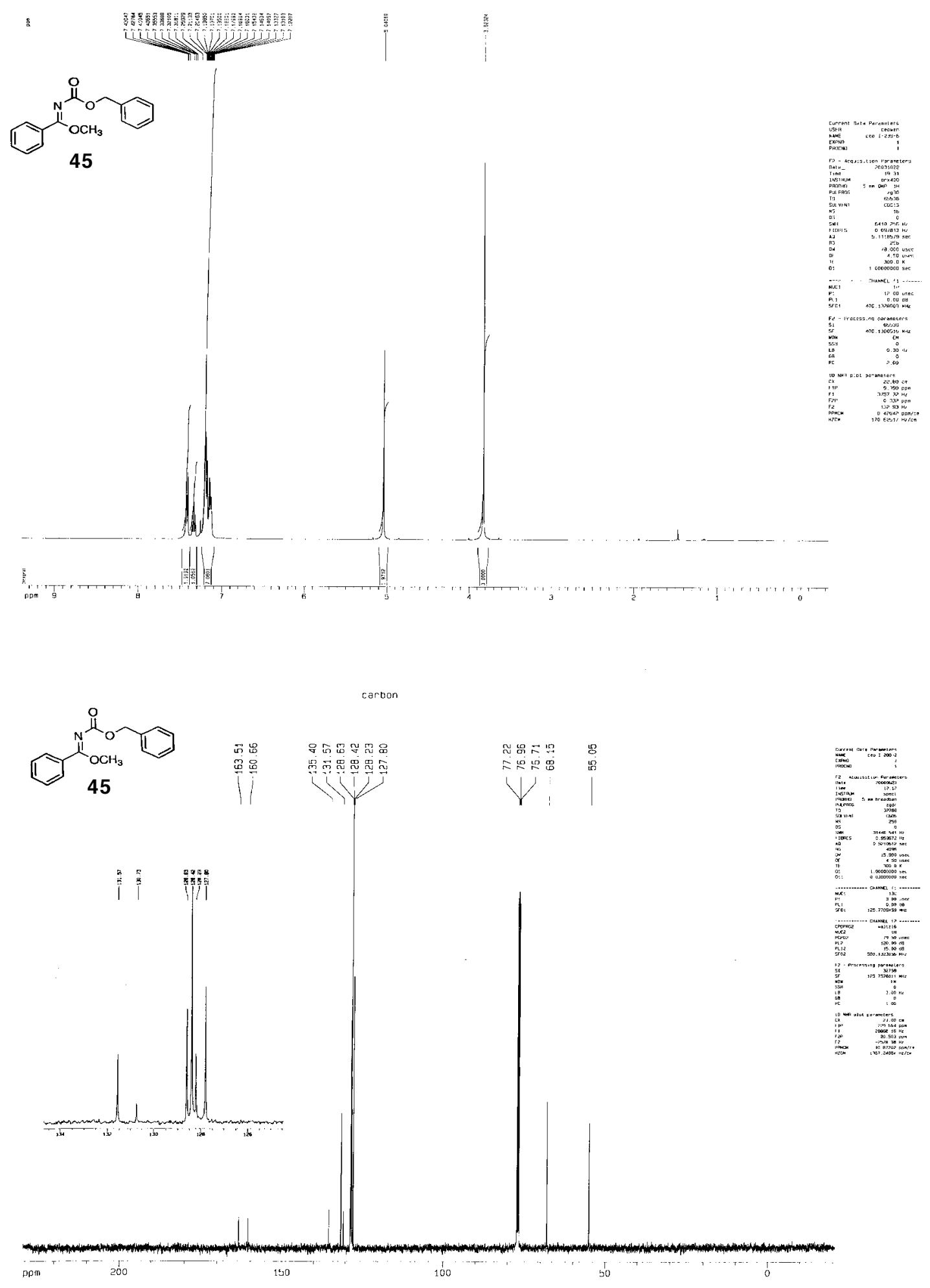

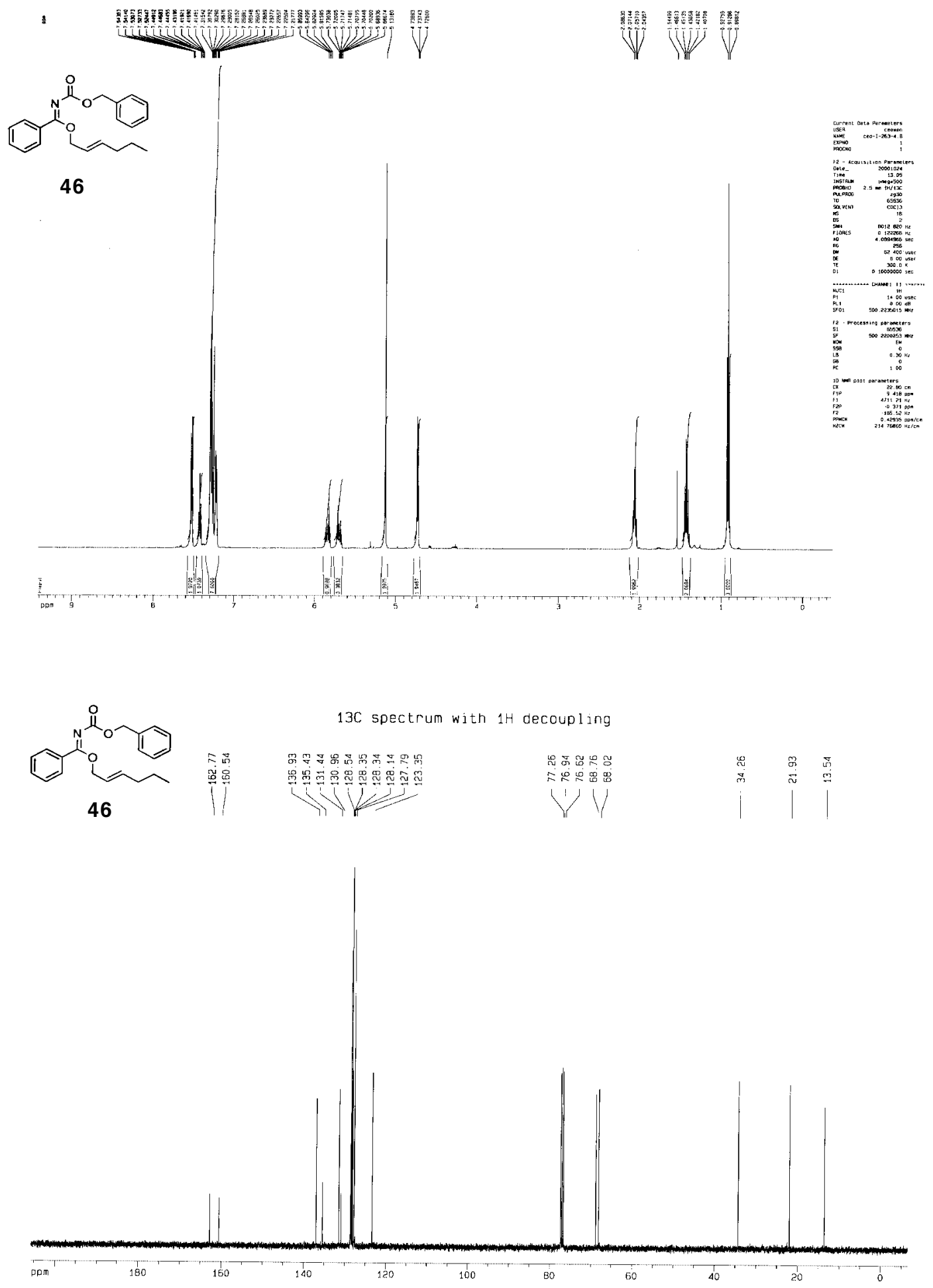

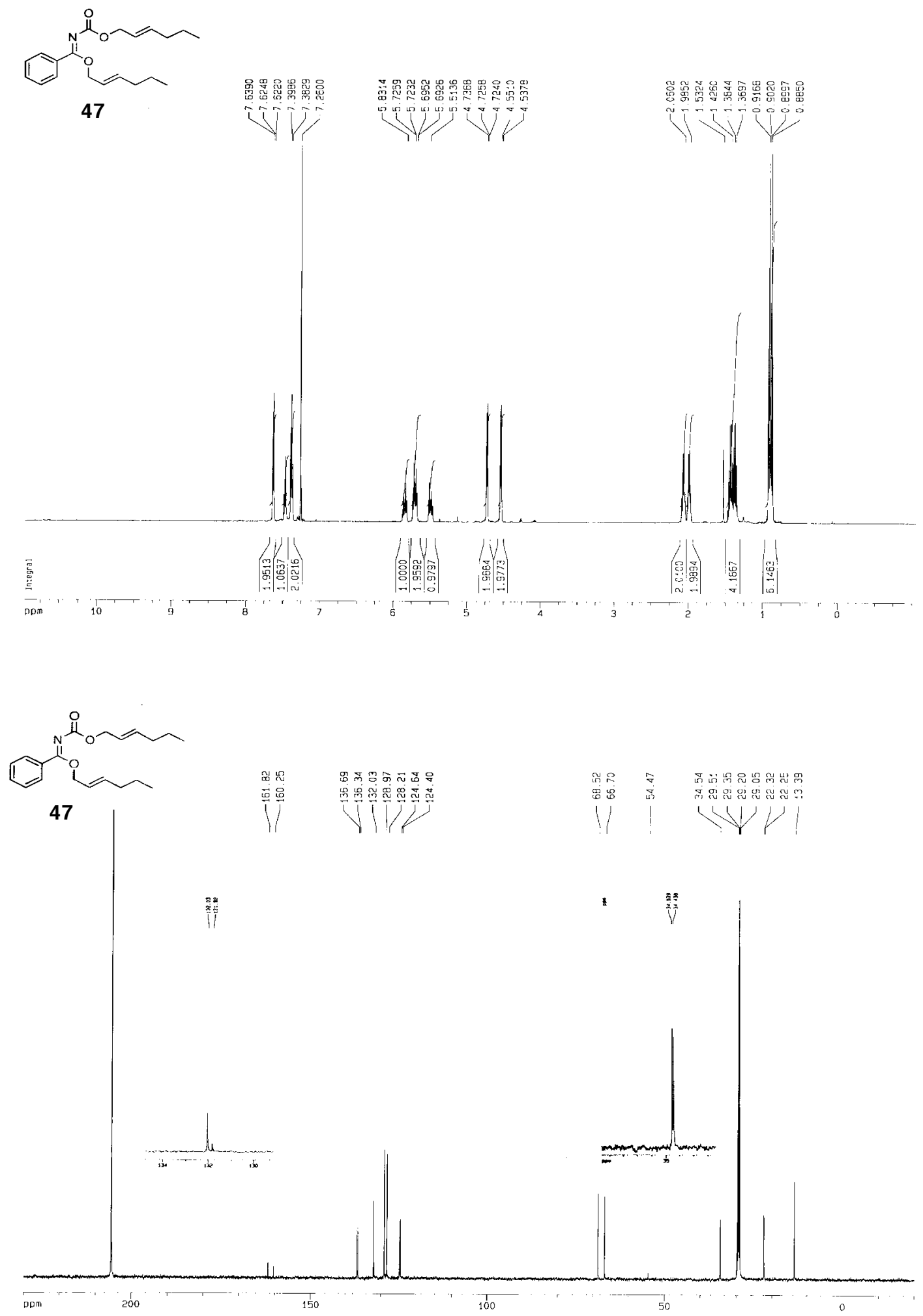

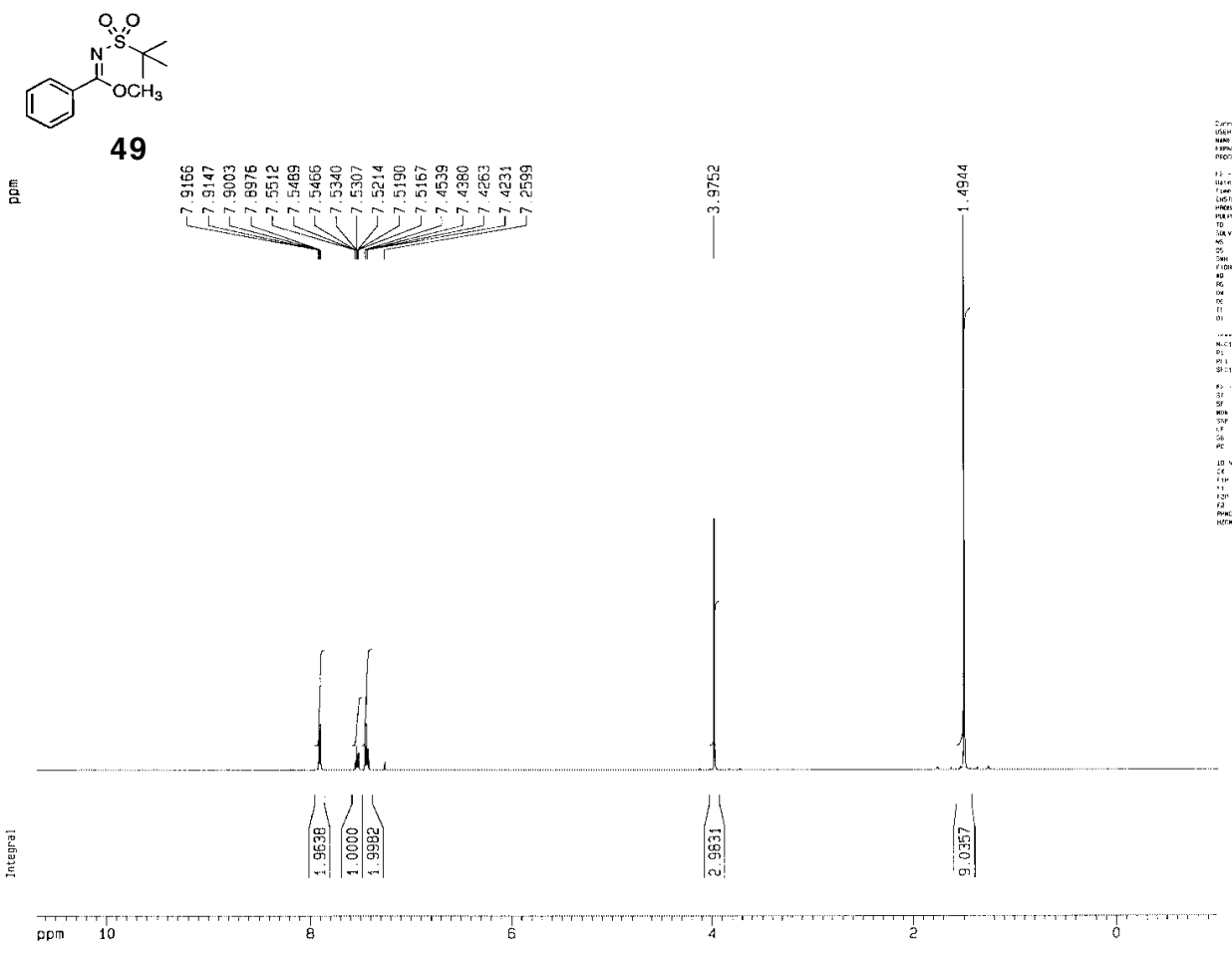

49

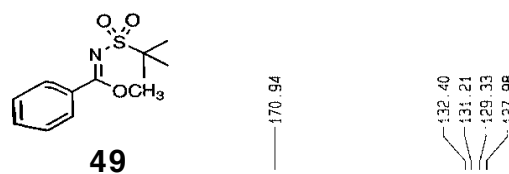

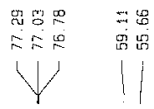
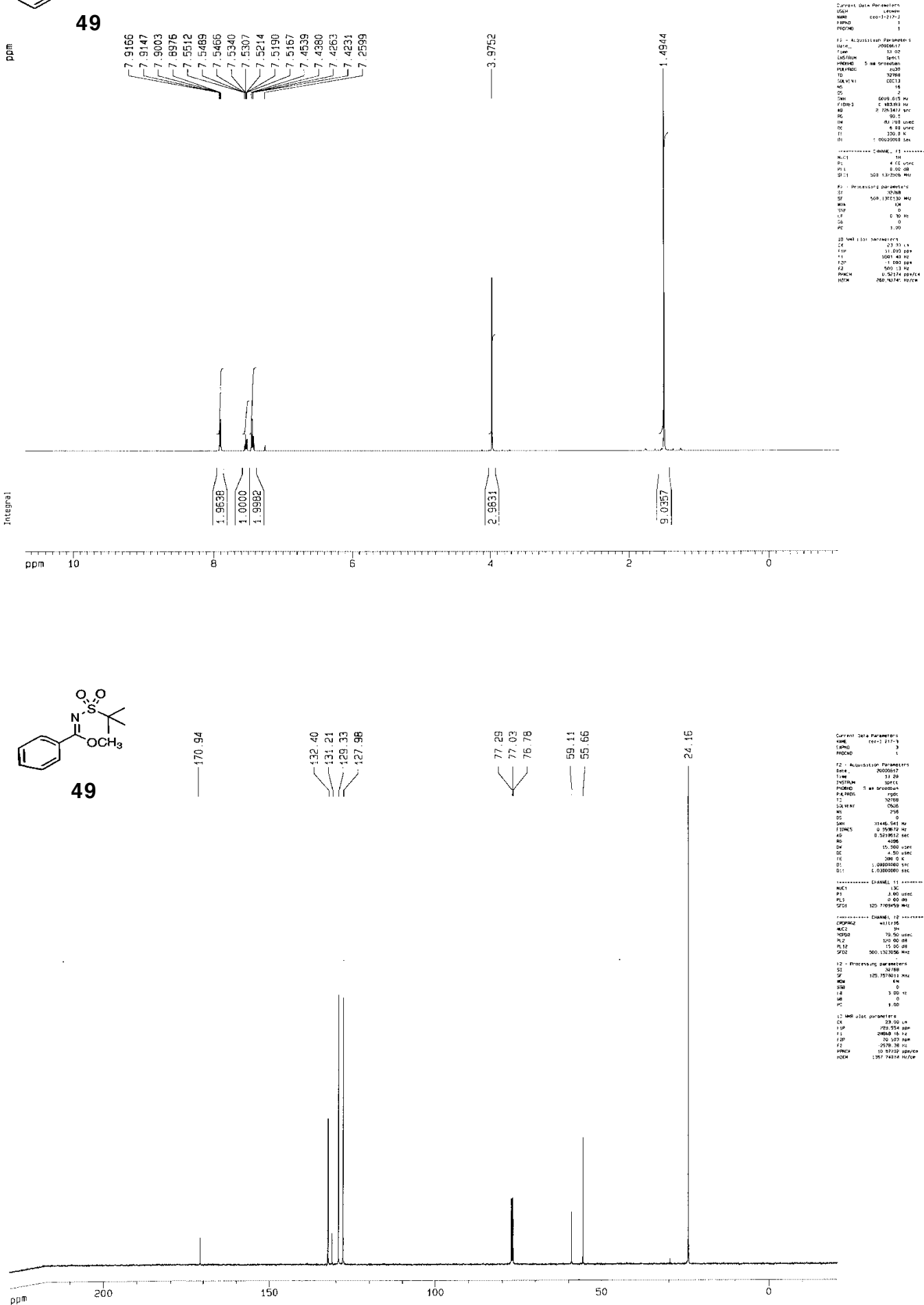

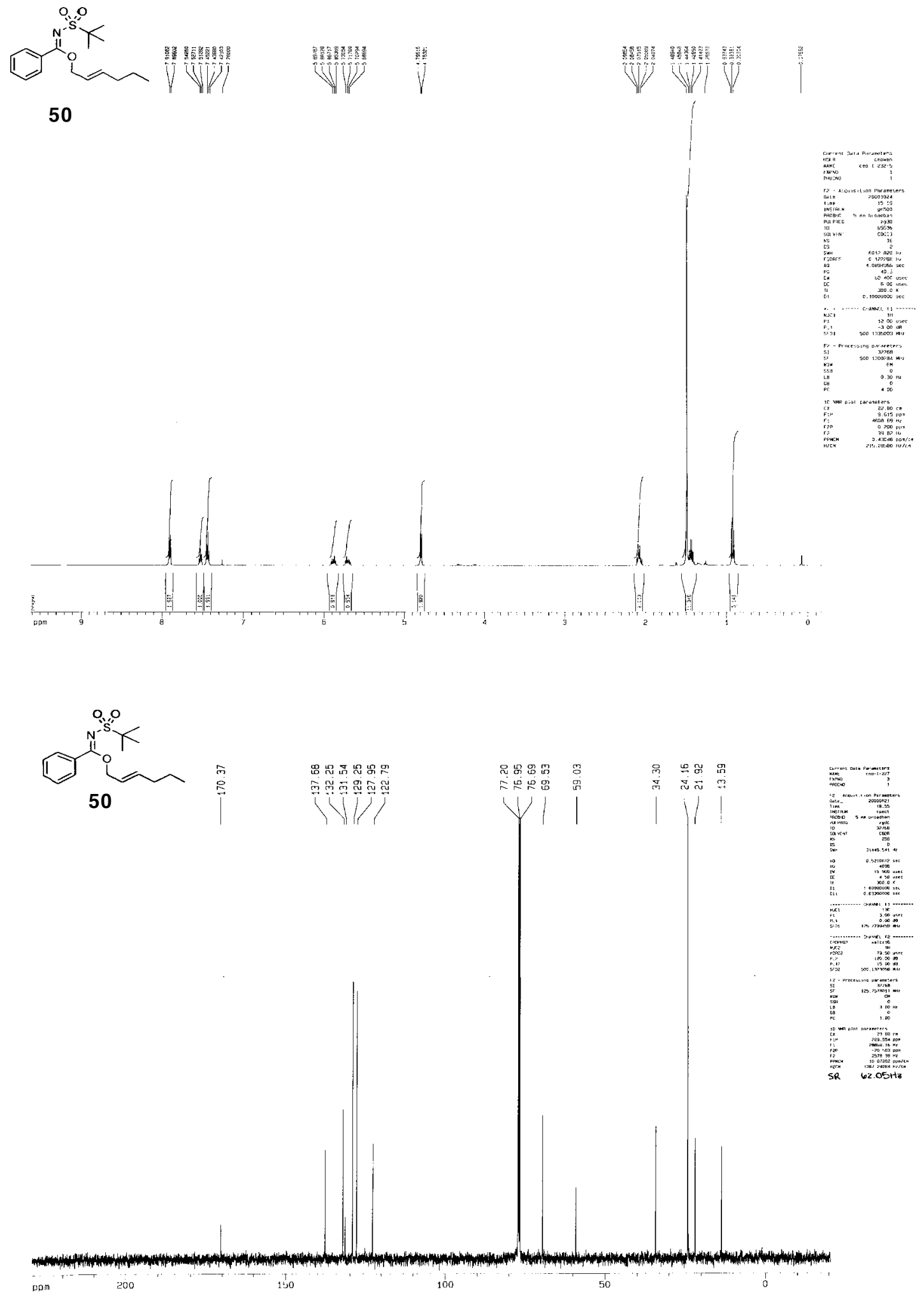

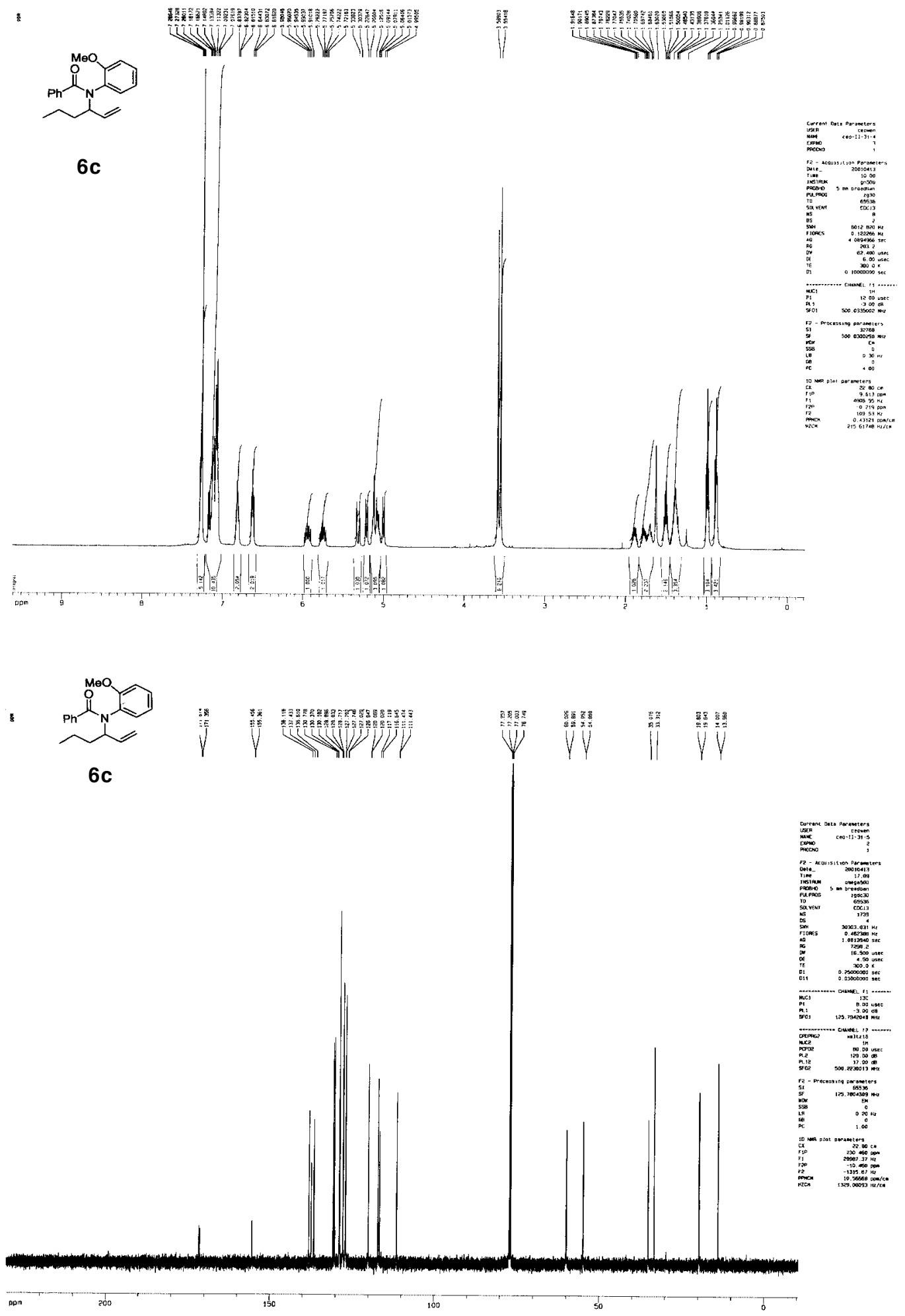

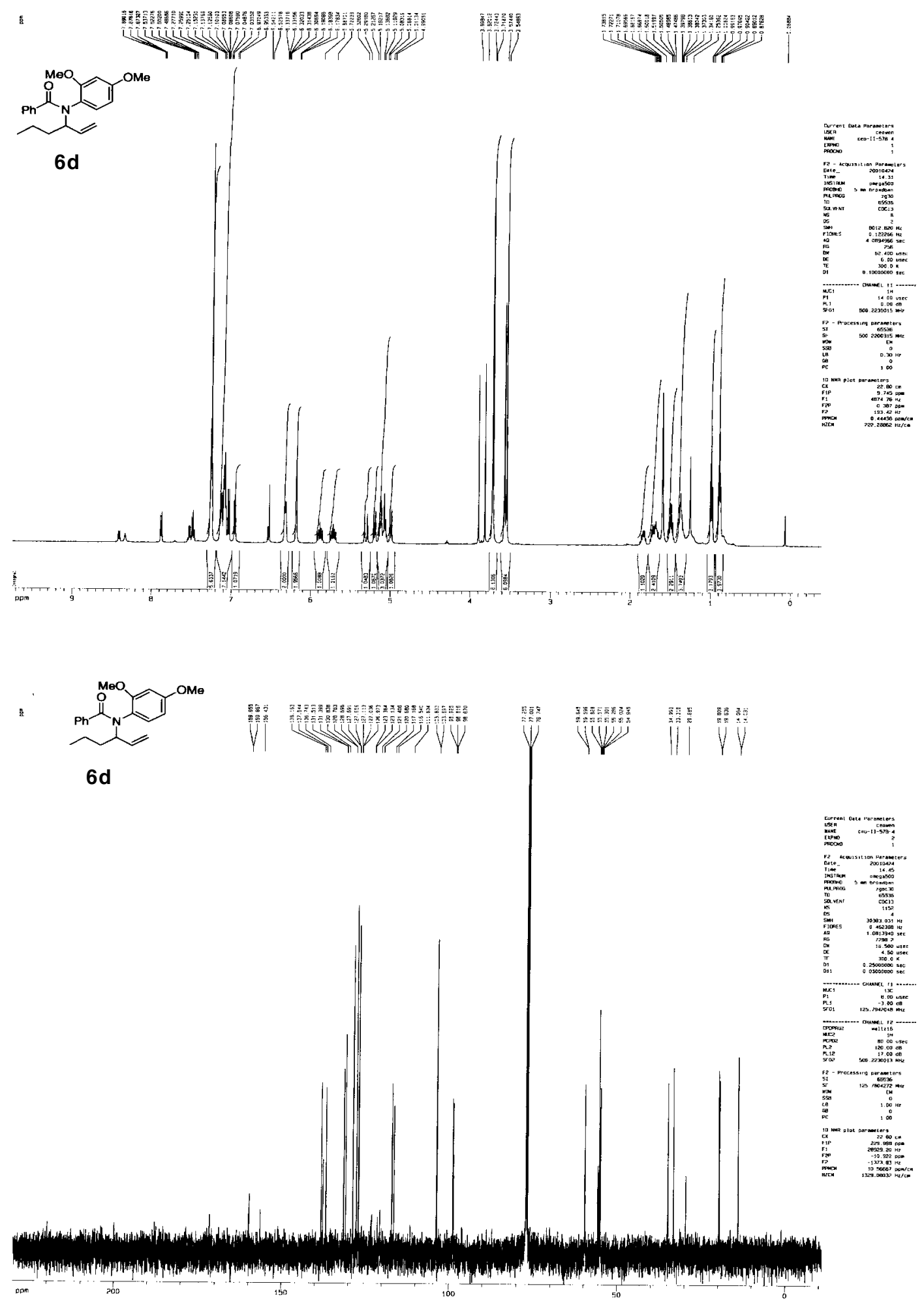

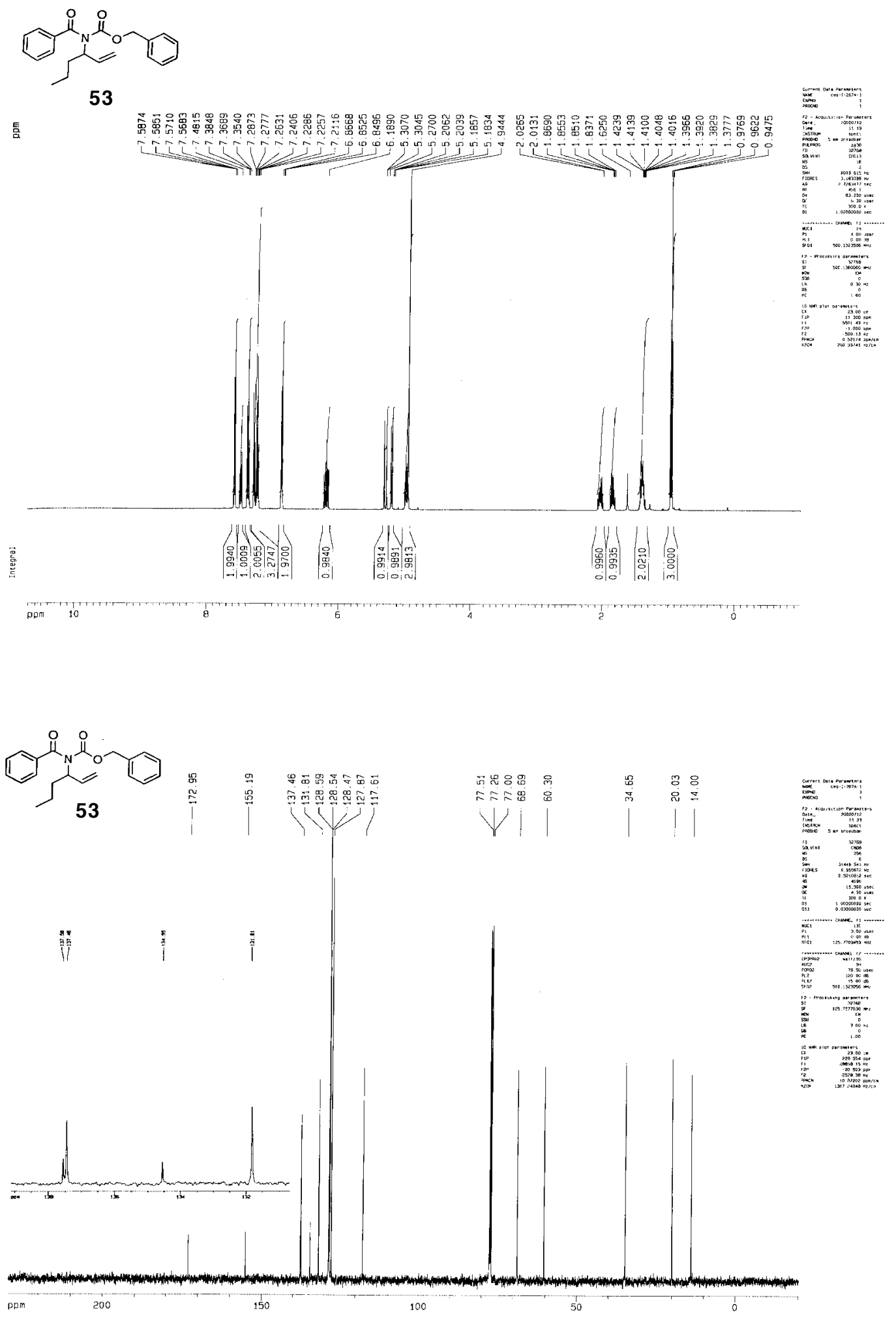


\section{Representative HPLC traces}
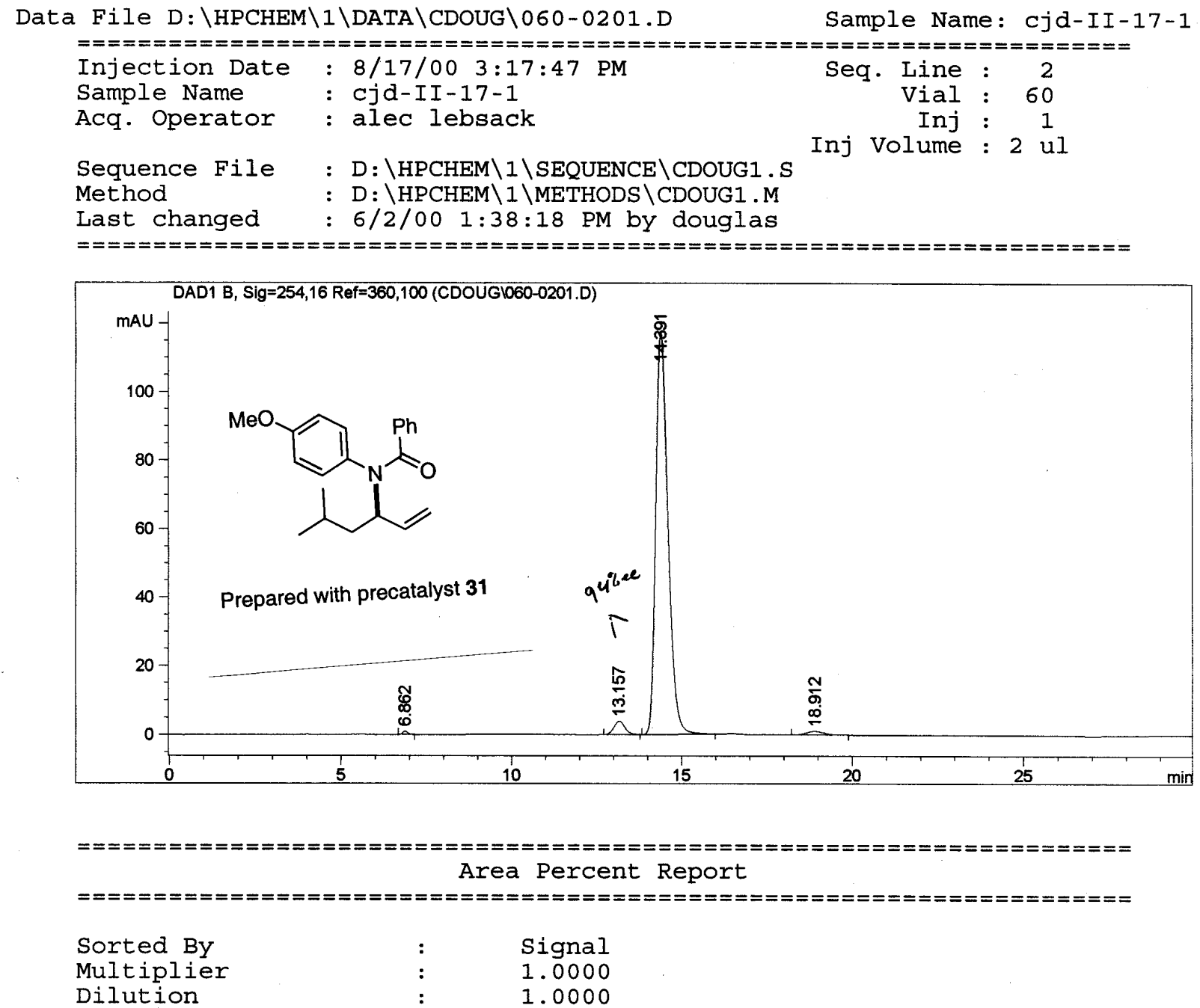

Signal 1: DAD1 B, Sig=254,16 $\operatorname{Ref}=360,100$

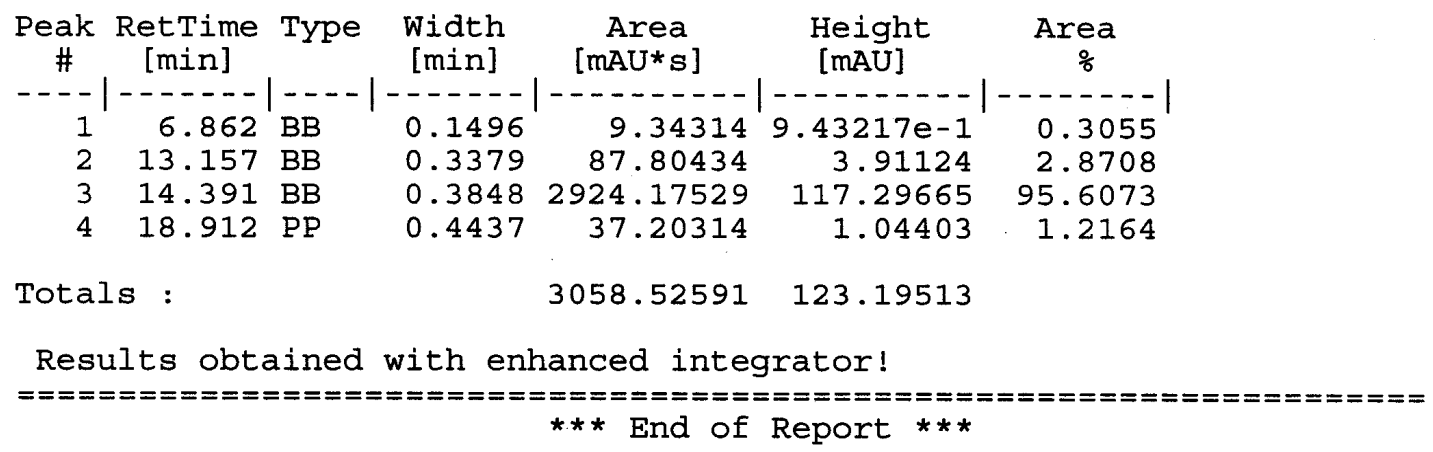



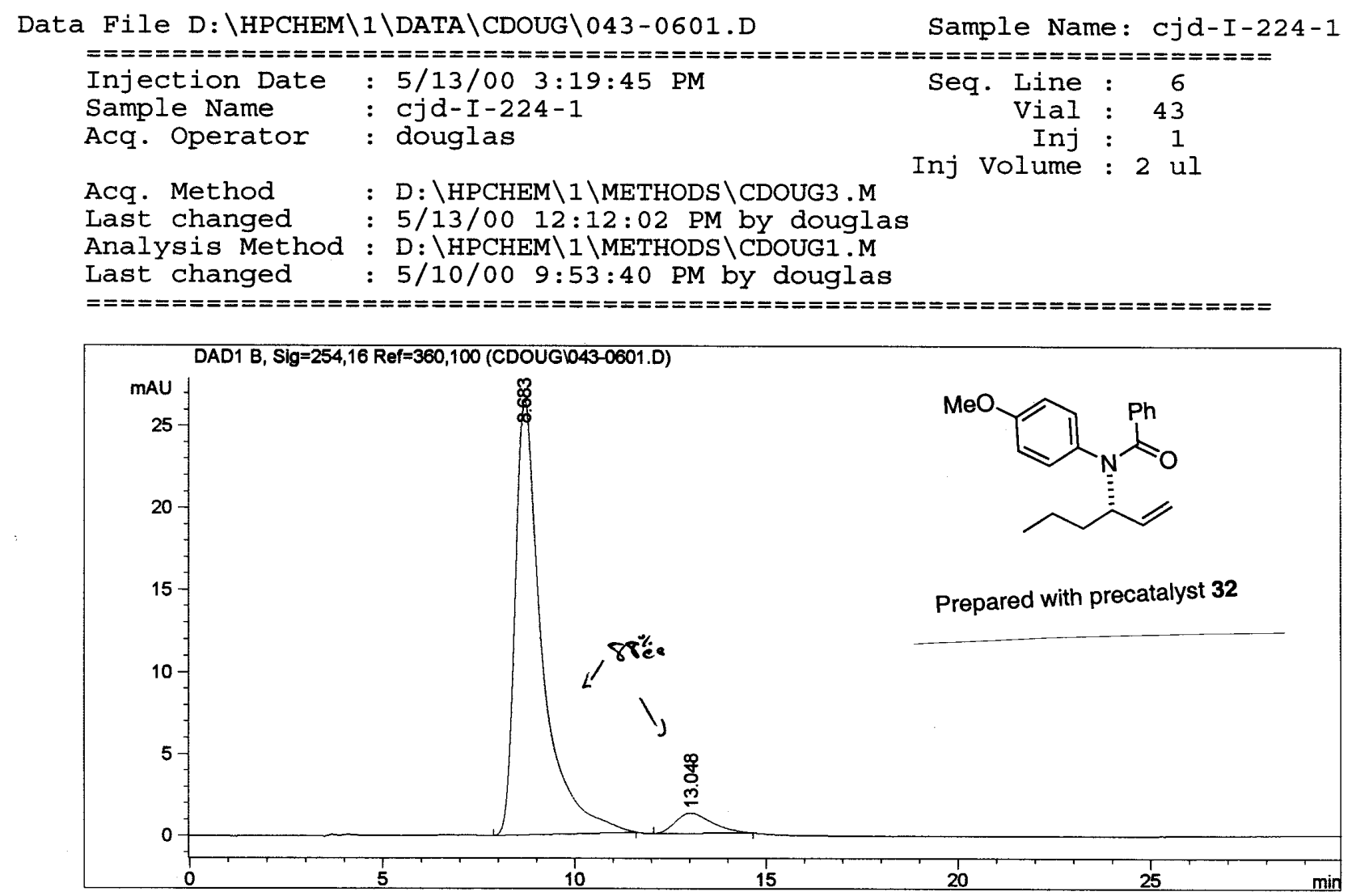

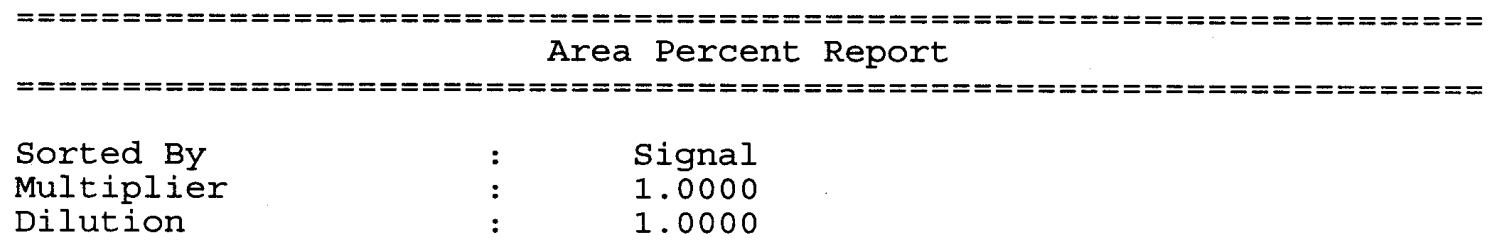

Signal 1: DAD1 B, Sig=254,16 Ref $=360,100$

\begin{tabular}{|c|c|c|c|c|c|c|}
\hline $\begin{array}{c}\text { Peak } \\
\#\end{array}$ & $\begin{array}{l}\text { RetTime } \\
\text { [min] }\end{array}$ & Type & $\begin{array}{l}\text { Width } \\
\text { [min] }\end{array}$ & $\begin{array}{c}\text { Area } \\
{\left[\mathrm{mAU}{ }^{*} \mathrm{~s}\right]}\end{array}$ & $\begin{array}{l}\text { Height } \\
\text { [mAU] }\end{array}$ & $\begin{array}{c}\text { Area } \\
\frac{\circ}{b}\end{array}$ \\
\hline $\begin{array}{l}1 \\
2\end{array}$ & $\begin{array}{r}8.683 \\
13.048\end{array}$ & $\begin{array}{l}\mathrm{BB} \\
\mathrm{PB}\end{array}$ & $\begin{array}{l}0.7099 \\
0.8041\end{array}$ & $\begin{array}{r}1305.41296 \\
84.95343\end{array}$ & $\begin{array}{r}26.53867 \\
1.24806\end{array}$ & $\begin{array}{r}93.8899 \\
6.1101\end{array}$ \\
\hline
\end{tabular}

Totals :

1390.36639

27.78673

Results obtained with enhanced integrator!

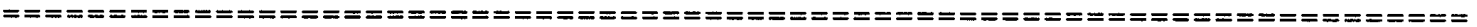
*** End of Report *** 

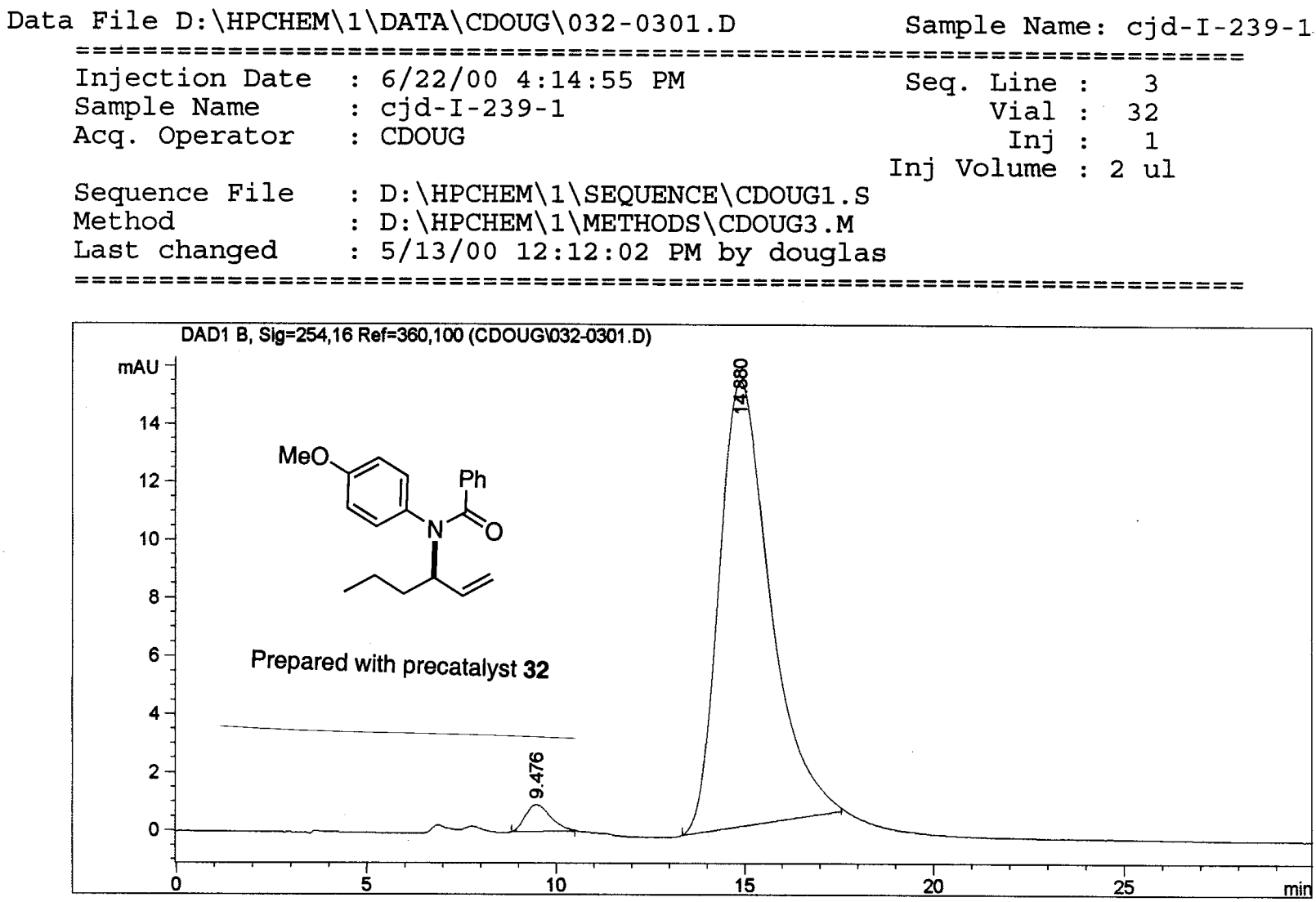

\begin{tabular}{|c|c|c|}
\hline Sorted By & : & Signal \\
\hline Multiplier & : & 1.0000 \\
\hline Dilution & : & 1.0000 \\
\hline
\end{tabular}

Signal 1: DAD1 B, Sig=254,16 Ref $=360,100$

\begin{tabular}{|c|c|c|c|c|c|c|}
\hline $\begin{array}{c}\text { Peak } \\
\quad \#\end{array}$ & $\begin{array}{l}\text { RetTime } \\
\text { [min] }\end{array}$ & Type & $\begin{array}{l}\text { Width } \\
\text { [min] }\end{array}$ & $\begin{array}{c}\text { Area } \\
{\left[\mathrm{mAU}{ }^{*} \mathrm{~s}\right]}\end{array}$ & $\begin{array}{l}\text { Height } \\
\text { [mAU] }\end{array}$ & $\begin{array}{c}\text { Area } \\
\text { o }\end{array}$ \\
\hline & ----- & & ------- & $\ldots \ldots$ & ------- & ------ \\
\hline $\begin{array}{l}1 \\
2\end{array}$ & $\begin{array}{r}9.476 \\
14.880\end{array}$ & $\begin{array}{l}\mathrm{BB} \\
\mathrm{BB}\end{array}$ & $\begin{array}{l}0.5642 \\
1.3205\end{array}$ & $\begin{array}{r}44.14556 \\
1442.71265\end{array}$ & $\begin{array}{r}9.36113 e-1 \\
15.35027\end{array}$ & $\begin{array}{r}2.9690 \\
97.0310\end{array}$ \\
\hline & : & & & 1486.85820 & 16.28638 & \\
\hline
\end{tabular}

Results obtained with enhanced integrator!

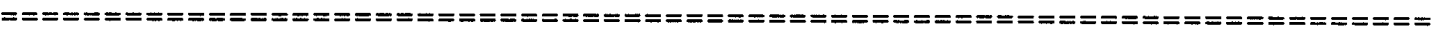
*** End of Report *** 

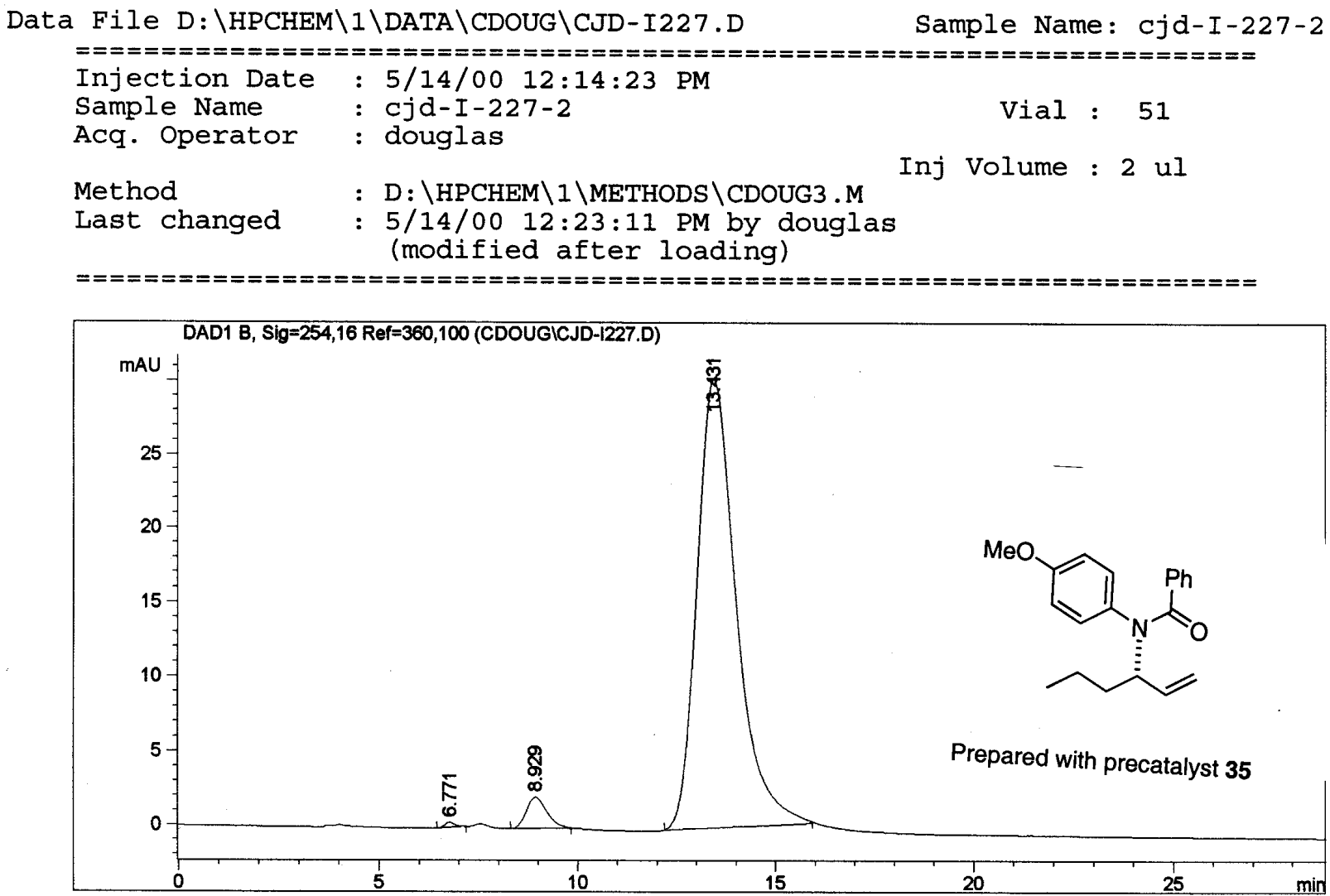

\begin{tabular}{|c|c|c|}
\hline Sorted By & : & Signal \\
\hline Multiplier & : & 1.0000 \\
\hline Dilution & : & 1.000 \\
\hline
\end{tabular}

Signal 1: DAD1 B, Sig=254,16 $\operatorname{Ref}=360,100$

\begin{tabular}{|c|c|c|c|c|c|c|}
\hline $\begin{array}{c}\text { Peak } \\
\#\end{array}$ & $\begin{array}{l}\text { RetTime } \\
\text { [min] }\end{array}$ & Type & $\begin{array}{l}\text { Width } \\
\text { [min] }\end{array}$ & $\begin{array}{c}\text { Area } \\
{\left[\mathrm{mAU} \mathbf{U}^{*} \mathrm{~s}\right]}\end{array}$ & $\begin{array}{l}\text { Height } \\
\text { [mAU] }\end{array}$ & $\begin{array}{c}\text { Area } \\
\text { \% }\end{array}$ \\
\hline $\begin{array}{r}--- \\
1 \\
2 \\
3\end{array}$ & $\begin{array}{r}6.771 \\
8.929 \\
13.431\end{array}$ & $\begin{array}{l}-- \\
\mathrm{PB} \\
\mathrm{BB} \\
\mathrm{BB}\end{array}$ & $\begin{array}{l}0.2307 \\
0.4968 \\
1.0091\end{array}$ & $\begin{array}{r}6.03377 \\
77.34119 \\
2090.67749\end{array}$ & $\begin{array}{r}3.47254 \mathrm{e}-1 \\
2.12420 \\
30.37284\end{array}$ & $\begin{array}{r}0.2775 \\
3.5575 \\
96.1650\end{array}$ \\
\hline Tota & : & & & 2174.05246 & 32.84429 & \\
\hline
\end{tabular}

Results obtained with enhanced integrator!

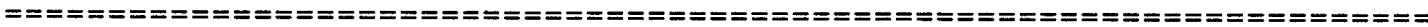
$\star * *$ End of Report $* * *$ 

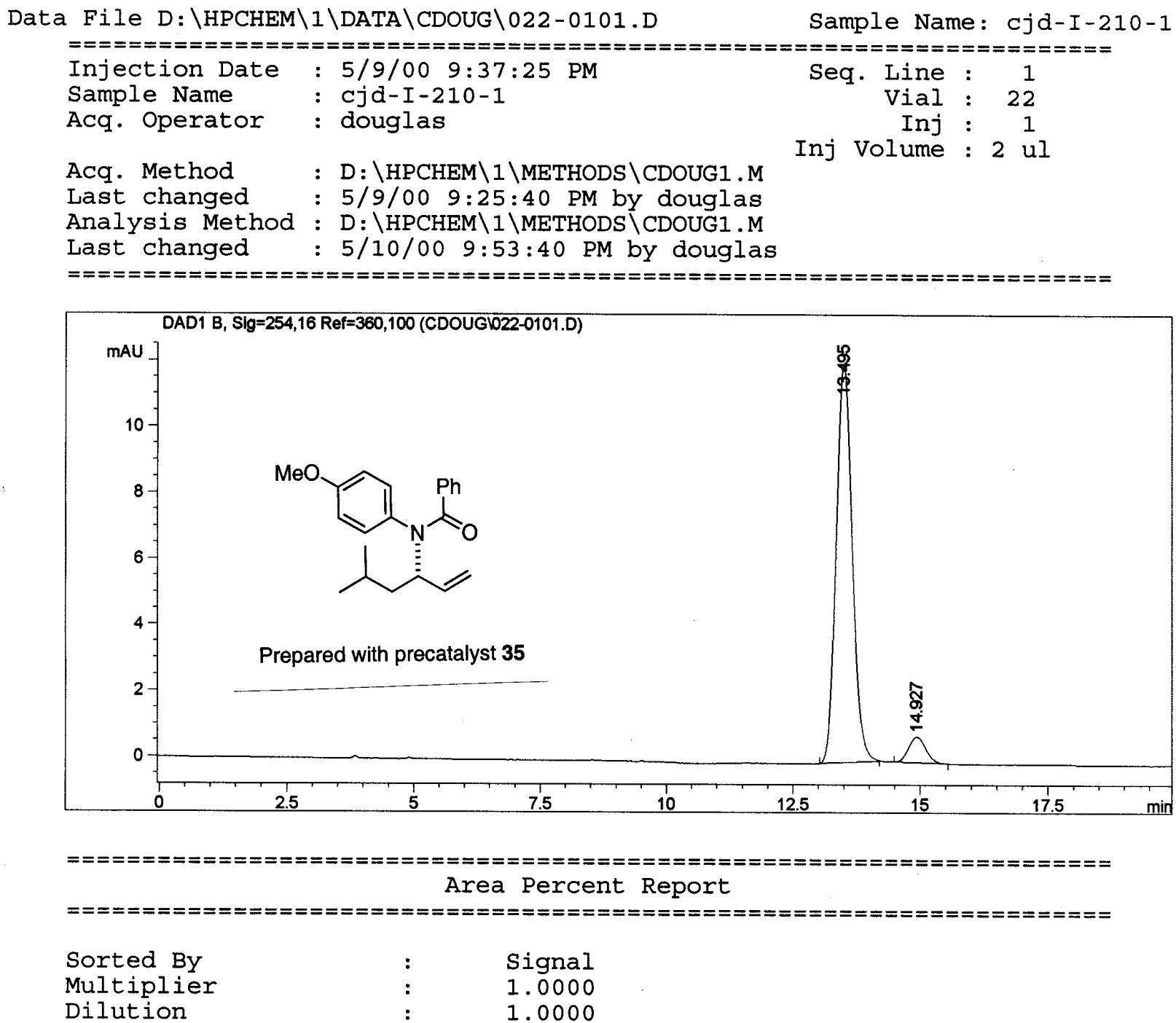

Signal 1: DAD1 B, Sig=254,16 Ref $=360,100$

\begin{tabular}{|c|c|c|c|c|c|c|}
\hline $\begin{array}{c}\text { Peak } \\
\#\end{array}$ & $\begin{array}{l}\text { RetTime } \\
\text { [min] }\end{array}$ & Type & $\begin{array}{l}\text { Width } \\
\text { [min] }\end{array}$ & $\begin{array}{c}\text { Area } \\
{\left[\mathrm{mAU}{ }^{*} \mathrm{~s}\right]}\end{array}$ & $\begin{array}{l}\text { Height } \\
\text { [mAU] }\end{array}$ & $\begin{array}{c}\text { Area } \\
\frac{q}{8}\end{array}$ \\
\hline $\begin{array}{l}1 \\
2\end{array}$ & $\begin{array}{l}13.495 \\
14.927\end{array}$ & $\begin{array}{l}\text { BB } \\
\text { BB }\end{array}$ & $\begin{array}{l}0.3392 \\
0.3434\end{array}$ & $\begin{array}{r}266.18753 \\
18.29179\end{array}$ & $\begin{array}{r}12.07376 \\
7.80630 \mathrm{e}-1\end{array}$ & $\begin{array}{r}93.5701 \\
6.4299\end{array}$ \\
\hline
\end{tabular}

$$
\text { Totals : } \quad 284.47932 \quad 12.85438
$$

Results obtained with enhanced integrator!

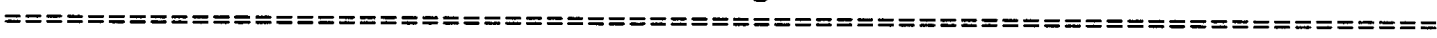
*** End of Report *** 

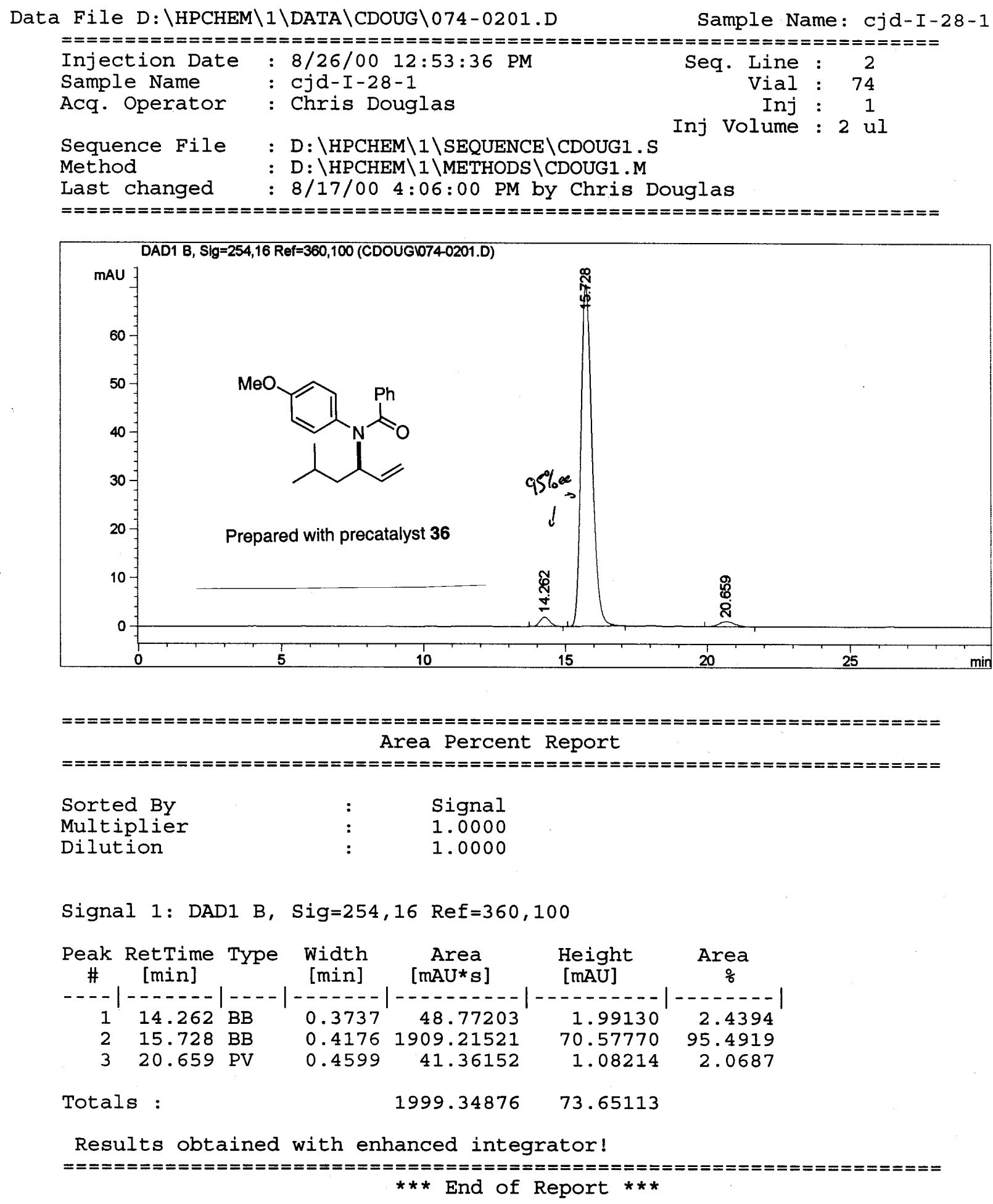